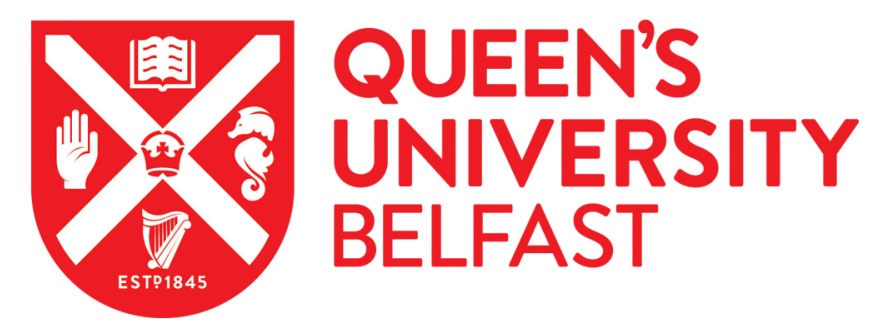

\title{
Measuring Dark Energy Properties with Photometrically Classified Pan-STARRS Supernovae. II. Cosmological Parameters
}

Jones, D. O., Scolnic, D. M., Riess, A. G., Rest, A., Kirshner, R. P., Berger, E., Kessler, R., Pan, Y. C., Foley, R. J., Chornock, R., Ortega, C. A., Challis, P. J., Burgett, W. S., Chambers, K. C., Draper, P. W., Flewelling, H., Huber, M. E., Kaiser, N., Kudritzki, R. P., ... Smith, K. W. (2018). Measuring Dark Energy Properties with Photometrically Classified Pan-STARRS Supernovae. II. Cosmological Parameters. Astrophysical Journal, 857(1), [51]. https://doi.org/10.3847/1538-4357/aab6b1

Published in:

Astrophysical Journal

Document Version:

Publisher's PDF, also known as Version of record

Queen's University Belfast - Research Portal:

Link to publication record in Queen's University Belfast Research Portal

Publisher rights

(C) 2018. The American Astronomical Society.This work is made available online in accordance with the publisher's policies. Please refer to any applicable terms of use of the publisher.

\section{General rights}

Copyright for the publications made accessible via the Queen's University Belfast Research Portal is retained by the author(s) and / or other copyright owners and it is a condition of accessing these publications that users recognise and abide by the legal requirements associated with these rights.

Take down policy

The Research Portal is Queen's institutional repository that provides access to Queen's research output. Every effort has been made to ensure that content in the Research Portal does not infringe any person's rights, or applicable UK laws. If you discover content in the Research Portal that you believe breaches copyright or violates any law, please contact openaccess@qub.ac.uk. 


\title{
Measuring Dark Energy Properties with Photometrically Classified Pan-STARRS Supernovae. II. Cosmological Parameters
}

\author{
D. O. Jones ${ }^{1}$ (10, D. M. Scolnic ${ }^{2,13}$, A. G. Riess ${ }^{3,4}$, A. Rest ${ }^{3,4}$, R. P. Kirshner ${ }^{5,6}$, E. Berger ${ }^{5}$ (1), R. Kessler ${ }^{2}$ (1), Y.-C. Pan ${ }^{1}$, \\ R. J. Foley ${ }^{1}$, R. Chornock ${ }^{7}$, C. A. Ortega ${ }^{3}$, P. J. Challis ${ }^{5}$, W. S. Burgett ${ }^{8}$ (iD, K. C. Chambers ${ }^{8}$ (D), P. W. Draper ${ }^{9}$ (iD), H. Flewelling ${ }^{8}$ (D), \\ M. E. Huber ${ }^{8}$ (D) N. Kaiser ${ }^{8}$ (D) , R.-P. Kudritzki ${ }^{8}$, N. Metcalfe ${ }^{9}$ (D) , J. Tonry ${ }^{8}$ (D) R. J. Wainscoat ${ }^{8}$ (D), C. Waters ${ }^{8}$ (D) E. E. E. Gall ${ }^{10,11^{\prime}}$,

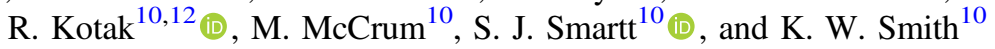 \\ ${ }^{1}$ Department of Astronomy and Astrophysics, University of California, Santa Cruz, CA 92064, USA; david.jones@ucsc.edu \\ ${ }^{2}$ University of Chicago, Kavli Institute for Cosmological Physics, Chicago, IL, USA \\ ${ }^{3}$ Department of Physics and Astronomy, The Johns Hopkins University, Baltimore, MD 21218, USA \\ ${ }^{4}$ Space Telescope Science Institute, Baltimore, MD 21218, USA \\ ${ }^{5}$ Harvard-Smithsonian Center for Astrophysics, 60 Garden Street, Cambridge, MA 02138, USA \\ ${ }^{6}$ Department of Physics, Harvard University, Cambridge, MA 02138, USA \\ ${ }^{7}$ Astrophysical Institute, Department of Physics and Astronomy, 251B Clippinger Lab, Ohio University, Athens, OH 45701, USA \\ 8 Institute for Astronomy, University of Hawaii at Manoa, Honolulu, HI 96822, USA \\ ${ }^{9}$ Department of Physics, Durham University, South Road, Durham DH1 3LE, UK
${ }^{10}$ Astrophysics Research Centre, School of Mathematics and Physics, Queen's University Belfast, Belfast BT7 1NN, UK \\ ${ }_{11}$ Max-Planck-Institut für Astrophysik, Karl-Schwarzschild-Str. 1, D-85748 Garching-bei-München, Germany \\ ${ }^{12}$ Tuorlan Observatorio, Väisäläntie 20, FI-21500 Piikkiö, Finland \\ Received 2017 October 2; revised 2018 March 9; accepted 2018 March 12; published 2018 April 12
}

\begin{abstract}
We use 1169 Pan-STARRS supernovae (SNe) and 195 low- $z(z<0.1)$ SNe Ia to measure cosmological parameters. Though most Pan-STARRS SNe lack spectroscopic classifications, in a previous paper we demonstrated that photometrically classified $\mathrm{SNe}$ can be used to infer unbiased cosmological parameters by using a Bayesian methodology that marginalizes over core-collapse (CC) SN contamination. Our sample contains nearly twice as many SNe as the largest previous SN Ia compilation. Combining SNe with cosmic microwave background (CMB) constraints from Planck, we measure the dark energy equation-of-state parameter $w$ to be $-0.989 \pm 0.057$ (stat + sys). If $w$ evolves with redshift as $w(a)=w_{0}+w_{a}(1-a)$, we find $w_{0}=-0.912 \pm 0.149$ and $w_{a}=$ $-0.513 \pm 0.826$. These results are consistent with cosmological parameters from the Joint Light-curve Analysis and the Pantheon sample. We try four different photometric classification priors for Pan-STARRS SNe and two alternate ways of modeling CC SN contamination, finding that no variant gives a $w$ differing by more than $2 \%$ from the baseline measurement. The systematic uncertainty on $w$ due to marginalizing over CC SN contamination, $\sigma_{w}^{\mathrm{CC}}=0.012$, is the third-smallest source of systematic uncertainty in this work. We find limited $(1.6 \sigma)$ evidence for evolution of the SN color-luminosity relation with redshift, a possible systematic that could constitute a significant uncertainty in future high- $z$ analyses. Our data provide one of the best current constraints on $w$, demonstrating that samples with $\sim 5 \%$ CC SN contamination can give competitive cosmological constraints when the contaminating distribution is marginalized over in a Bayesian framework.
\end{abstract}

Key words: cosmology: observations - dark energy - supernovae: general

Supporting material: machine-readable tables

\section{Introduction}

The cause of the universe's accelerating expansion at late times is one of the fundamental questions in astrophysics today. Twenty years ago, distances from Type Ia supernovae (SNe Ia) revealed that the universe was accelerating (Riess et al. 1998; Perlmutter et al. 1999), and the most common interpretation of this cosmic acceleration was that $\sim 70 \%$ of the energy in the present-day universe must consist of a repulsive "dark energy." In the time since this discovery, large SN data sets have compiled up to $\sim 750$ spectroscopically confirmed SNe Ia and measured the expansion history of the universe at $z \lesssim 1$ with increasing precision (Riess et al. 2004; Hicken et al. 2009b; Kessler et al. 2009; Conley et al. 2011; Sullivan et al. 2011; Suzuki et al. 2012; Betoule et al. 2014; Rest et al. 2014). Because SNe Ia are observed in the recent cosmic epochs when dark energy is most dominant, they have more leverage to

\footnotetext{
${ }^{13}$ Hubble, KICP Fellow.
}

measure dark energy than most other cosmological probes (Weinberg et al. 2013). In conjunction with baryon acoustic oscillation (BAO) and cosmic microwave background (CMB) constraints (e.g., Bennett et al. 2003; Eisenstein et al. 2005; Anderson et al. 2014; Planck Collaboration et al. 2016a), $\mathrm{SNe}$ Ia can be used to infer the dark energy equation-of-state parameter $w$ (equal to $P / \rho c^{2}$, the ratio of pressure to density).

The simplest model of dark energy is a cosmological constant, a vacuum energy that exerts a spatially and temporally constant negative pressure $(w=-1)$. However, if $w$ is measured to be greater than -1 , it would be an indication of "quintessence" dark energy, a dynamic scalar field. A $w$ value of less than -1 would imply so-called "phantom" dark energy, which requires extremely exotic physics (Amendola et al. 2013).

Nearly all SN Ia analyses have measured a dark energy equation of state consistent with $w=-1$. The most precise measurement to date is that of Betoule et al. (2014, hereafter B14), who combined 740 spectroscopically confirmed SNe Ia from the Sloan Digital Sky Survey (SDSS; Alam et al. 2015), the 
SuperNova Legacy Survey (SNLS; Astier et al. 2006), high-z SNe from HST (Riess et al. 2007), and low-z SNe (Hamuy et al. 1996; Riess et al. 1999; Jha et al. 2006; Hicken et al. 2009a, 2009b; Contreras et al. 2010; Folatelli et al. 2010) to form the Joint Lightcurve Analysis (JLA). JLA SNe Ia, when combined with CMB data from the Planck satellite and BAO constraints from Anderson et al. (2014) and Ross et al. (2015), yield $w=-1.006 \pm 0.045$ (Planck Collaboration et al. 2016a).

Statistical and systematic uncertainties on the JLA measurement of $w$ are approximately equal. Though a great deal of recent progress has been made to lower systematic uncertainties, including the leading systematic of photometric calibration error (Scolnic et al. 2015), lower uncertainties are also possible just by adding more SNe Ia. Although a significant reduction of the statistical uncertainty now requires hundreds of additional SNe Ia, thousands of SNe Ia have already been discovered by Pan-STARRS (PS1; Kaiser et al. 2010). Thousands more are currently being discovered by the Dark Energy Survey (DES; Flaugher 2005), and tens or hundreds of thousands will be discovered by the Large Synoptic Survey Telescope (LSST) in the coming decade.

Obtaining spectroscopic classifications for thousands of SNe is prohibitively expensive. Spectra of SNe Ia cannot be efficiently obtained with multiobject spectroscopy, as they have a sparse density on the sky: their rate is $\sim 10 \mathrm{yr}^{-1} \mathrm{deg}^{-2}$ for those with $R \lesssim 22$, and spectral classifications must be obtained within $\sim 2$ weeks of maximum light. At the median PS1 redshift of $z \sim 0.3$, spectroscopic classifications also necessitate $\sim 1 \mathrm{hr}$ or more of $4 \mathrm{~m}$ class telescope time per SN. In addition, $\sim 30 \%$ of these $\mathrm{SNe}$ Ia will fail sample-selection requirements after their spectra have been observed and thus cannot be placed on the Hubble diagram (Section 2.1). Assuming poor weather on $\sim 30 \%-50 \%$ of nights, 100 nights of $4 \mathrm{~m}$ class telescope time will result in a cosmologically useful sample of just $\sim 400 \mathrm{SNe}$ Ia. In future surveys, such as LSST, the cost of obtaining spectroscopy for tens of thousands of SNe Ia will far exceed the available resources.

The alternative to spectroscopic classifications is using classifications based only on photometric SN light curves, but this method subjects the sample to contamination by corecollapse (CC) SNe and peculiar SNe Ia. However, if cosmological distances can be measured without bias in a sample with CC SN contamination, photometrically classified SNe Ia could be used to measure $w$ without penalty. To this end, SN lightcurve classification algorithms have improved greatly in the last few years. The advent of LSST has provided additional motivation to develop quick, robust classification methods that rely only on limited photometric data (e.g., Saha et al. 2016). Machine-learning algorithms in particular have been found to yield both efficiencies (few bona fide $\mathrm{SNe}$ Ia are misclassified) and sample purities $\gtrsim 96 \%$ in cases where the classifier can be trained on a representative SN sample (Sako et al. 2014; Lochner et al. 2016).

The first measurement of $w$ with photometrically classified SNe, Campbell et al. (2013), used 752 SDSS SNe, most lacking spectroscopic classifications, to measure cosmological parameters. They reduced CC SN contamination using the PSNID Bayesian light-curve classifier (Sako et al. 2011), among other sample cuts, and estimated that their final sample had $3.9 \%$ CC SN contamination. However, Campbell et al. (2013) did not include a systematic uncertainty budget in their measurements. Because $\mathrm{CCSNe}$ are 1-2 mag fainter than
SNe Ia, a contamination fraction of just $2 \%$ could shift the mean distance by $0.02-0.04 \mathrm{mag}$, equivalent to a $5 \%-10 \%$ difference in $w$ over the redshift range $0<z<0.5$.

For this reason, Kunz et al. (2007) proposed the Bayesian Estimation Applied to Multiple Species (BEAMS) method to simultaneously determine the SN Ia and CC SN distributions. BEAMS models photometrically selected $\mathrm{SN}$ samples as a combination of SNe Ia and CC SNe, simultaneously fits for the contributions of each, and marginalizes over nuisance parameters to give cosmological parameter measurements. BEAMS is able to yield cosmological parameter measurements with less bias and nearly optimal uncertainties (Kunz et al. 2007). Hlozek et al. (2012), the first measurement of cosmological parameters from photometrically classified SNe, used the BEAMS method to measure the cosmic matter density $\Omega_{M}$ from SDSS SNe lacking spectroscopic classifications, but again did not include a systematic uncertainty budget in their measurements. However, the case of systematic uncertainties in BEAMS was explored theoretically by Knights et al. (2013), who developed a BEAMS formalism for correlated $\mathrm{SN}$ data that gives a reliable cosmological parameter estimation (see also Rubin et al. 2015 for a treatment of systematic uncertainties that includes CC SN contamination).

We expanded on this work in Jones et al. (2017, hereafter J17). J17 undertook a series of Monte Carlo (MC) simulations to test the application of a BEAMS-like algorithm to a PS1 photometrically classified SN sample and made a first estimate of the systematic uncertainty on $w$ due to CCSN contamination. We found a statistically insignificant bias of $\Delta_{w}^{\mathrm{CC}}=-0.001 \pm 0.004$ and a modest systematic uncertainty of 0.014, which we estimated using four different SN classification methods and three different contamination models. J17 also included SN selection effects (i.e., Malmquist bias), which were not included in the original BEAMS analyses.

In the current work, we apply the J17 methodology to PS1 $\mathrm{SNe}$ to measure cosmological parameters with robust systematic uncertainties. Previously, only $10 \%$ of PS1 SNe Ia-half of the spectroscopically classified SN Ia sample-had been used to measure cosmological parameters (Rest et al. 2014; Scolnic et al. 2014). The present sample is drawn from 350 spectroscopically classified SNe Ia and 3261 PS1 SNe with spectroscopic host galaxy redshifts. We anchor our Hubble diagram with a compilation of spectroscopically confirmed low- $z$ SNe Ia from the CfA1-4 and Carnegie Supernova Project (CSP) samples (Riess et al. 1999; Jha et al. 2006; Hicken et al. 2009a, 2009b; Contreras et al. 2010; Folatelli et al. 2010; Stritzinger et al. 2011). We exclude SDSS and SNLS SNe from this sample in order to give cosmological constraints that are independent of previous high- $z$ data. After applying conventional light-curve cuts (e.g., B14), we will show that 1364 PS1+low-z SNe remain. Statistically, we expect $\sim 5 \%$ of these SNe to be CC SN contaminants (J17).

A companion paper, Scolnic et al. (2017, hereafter S17), compiles 1049 spectroscopically classified SNe Ia from PS1 and other surveys to give cosmological constraints. S17 presents the PS1 spectroscopic sample, including improvements to the PS1 photometric pipeline that are used in this work. This work also relies heavily on the detailed analysis and simulations of the low$z$ sample in S17 and their improvements to the relative and absolute photometric calibration of all surveys.

The sample of PS1 SNe with host galaxy redshifts was presented in $\mathrm{J} 17$, including a description of our campaign to 
measure host galaxy redshifts for $\sim 60 \%$ of all SN candidates. In Section 2.1, we briefly discuss this sample and present the low- $z$ and PS1 spectroscopically classified SNe that are included in this analysis. We also derive bias-corrected distance measurements and estimate the probability that each $\mathrm{SN}$ is Type Ia. In Section 3, we summarize our cosmological parameter estimation methodology, and in Section 4, we discuss contributions to the systematic uncertainty budget. In Section 5, we perform consistency checks on the methodology. In Section 6, we give measurements of $\Omega_{M}$ and $w$ from SN Ia+CMB constraints. In Section 7, we present combined cosmological constraints after combining $\mathrm{SNe}$ with $\mathrm{CMB}, \mathrm{BAO}$, and local $\mathrm{H}_{0}$ measurements and compare our constraints to those of B14 and S17. In Section 8, we examine the test case of measuring $w$ from a SN sample without any $z>0.1$ spectroscopic classifications. Our conclusions are in Section 9.

\section{Distances and Photometric Classifications from the SN Data}

\subsection{Data}

The PS1 Medium Deep Survey covers $107 \mathrm{deg}^{2}$ fields in five filters, with typical observing cadences in a given field of six observations per 10 days. The PS1 SN discovery pipeline is described in detail in Rest et al. (2014). Likely SNe were flagged based on three signal-to-noise ratio $(S / N) \geqslant 4$ observations in the $\operatorname{griz}_{P S 1}$ filters and no previous detection of a SN at that position. The PS1 survey overview is given in Chambers et al. (2016).

Over its $4 \mathrm{yr}$ of operation, PS1 flagged $\sim 5,200$ likely SNe. Spectroscopic follow-up was triggered for $\sim 10 \%$ of $\mathrm{SNe}$, typically those with $r \lesssim 22 \mathrm{mag}$, on a wide variety of spectroscopic instruments (see Rest et al. 2014 and S17). For 520 of these candidates, spectroscopic observations of the $\mathrm{SN}$ near maximum light allowed their type to be determined, and approximately 350 of these 520 were spectroscopically classified as Type Ia (S17).

During the last year of PS1, we began a survey to obtain spectroscopic host galaxy redshifts for the majority of the sample, both those with SN spectra and those without. This survey primarily used the Hectospec multifiber instrument on the MMT (Fabricant et al. 2005; Mink et al. 2007). We also measured redshifts with the Apache Point Observatory (APO) $3.5 \mathrm{~m}$ telescope ${ }^{14}$ the WIYN telescope, ${ }^{15}$ and, for two of the most southern medium-deep fields, the Anglo-Australian Telescope (AAT). An additional $\sim 600$ of our redshifts came from SDSS (Smee et al. 2013) or other public redshift surveys. ${ }^{16} \mathrm{We}$ chose targets independent of SN type in order to build a sample without any color or shape selection bias. Of 3930 targets, the host galaxies of $3261 \mathrm{SN}$ candidates had strong enough spectral features and high enough $\mathrm{S} / \mathrm{N}$ to yield reliable spectroscopic redshifts. These data are discussed in detail in $\mathrm{J} 17$. We estimate that $1.4 \%$ of these redshifts are incorrect, and, as $\mathrm{SNe}$ with incorrect redshifts are indistinguishable from CCSNe when placed on the Hubble diagram, the incorrect-redshift fraction will

\footnotetext{
${ }^{14}$ http://www.apo.nmsu.edu/arc35m/

15 The WIYN Observatory is a joint facility of the University of WisconsinMadison, Indiana University, the National Optical Astronomy Observatory, and the University of Missouri.

16 Public redshifts are from 2dFGRS (Colless et al. 2003), 6dFGS (Jones et al. 2009), DEEP2 (Newman et al. 2013), VIPERS (Scodeggio et al. 2018), VVDS (Le Fèvre et al. 2005), WiggleZ (Blake et al. 2008), and zCOSMOS (Lilly et al. 2007).
}

contribute to the "contamination" systematic uncertainty for this sample (also discussed in J17).

Though our sample contains a mix of galaxy types (and $\sim 25 \%$ of hosts are absorption-line galaxies), we are unable to obtain redshifts for $\mathrm{SNe}$ in low surface brightness hosts. Previous high- $z$ SN searches favored $\mathrm{SNe}$ in low surface brightness hosts, which allow SN spectra with less host galaxy contamination to be obtained. In the photometrically classified sample, however, including hostless SNe is impossible, and the hosts with spectroscopic redshifts have a median $r$ magnitude of 20.3. Therefore, the preponderance of bright, massive host galaxies gives our sample significantly different $\mathrm{SN}$ and host demographics compared to previous high $z$, data but makes it more similar to the nature of the current low- $z$ sample, which primarily consists of SNe Ia found by targeting bright galaxies.

After SN discovery and redshift follow-up, the PS1 light curves were reprocessed with an enhanced version of the discovery pipeline that included a more realistic (nonGaussian) point spread function (PSF) model. The PS1 photometric pipeline has been improved further for this analysis and the complementary analysis of S17. The improvements include deeper templates, more accurate astrometric alignment, and better PSF modeling. The zero-point calibration has also been improved by using the Ubercal process (Padmanabhan et al. 2008; Schlafly et al. 2012). Ubercal uses repeat observations of stars in PS1 to solve for the system throughput, atmospheric transparency, and detector flat field in the griz $_{P S 1}$ filters. It has a photometric accuracy of better than $1 \%$ over the entire PS1 $3 \pi$ survey area. Pipeline improvements are discussed in detail in S17.

We use a compilation of low- $z$ SNe observed over the last $\sim 20$ yr to anchor the Hubble diagram. Nearly all of these SNe are all included in the JLA analysis, including the CfA1-3 SN samples (Riess et al. 1999; Jha et al. 2006; Hicken et al. 2009a, 2009b) and CSP SNe from the first data release (Contreras et al. 2010; Folatelli et al. 2010). ${ }^{17}$ We exclude Calan/Tololo SNe (Hamuy et al. 1996), as most lie below the PS1 $3 \pi$ survey area and therefore cannot take advantage of the PS1-based photometric calibration system we use in this paper (Supercal; Scolnic et al. 2015). We also include the most recent CfA SN compilation (CfA4; Hicken et al. 2012) and the second CSP data release (Stritzinger et al. 2011), which were not included in the JLA analysis but are used in the Rest et al. (2014) and S17 PS1 cosmological analyses.

\subsection{SALT2 Model}

To derive distances from PS1 and low $-z$ SNe, we use the SALT2 light-curve model (Guy et al. 2010, hereafter G10) to measure the light-curve parameters of SNe Ia. We apply the most recent version of SALT2 (SALT2.4), which was retrained by $\mathrm{B} 14$ to include additional high- $z \mathrm{SNe}$ and improve the photometric calibration.

We then use the measured SALT2 light-curve parameters to restrict our sample to $\mathrm{SNe}$ with shapes and colors consistent with normal SNe Ia $\left(-0.3<c<0.3,-3<x_{1}<3\right)$ and well-measured shapes $\left(\sigma_{x_{1}}<1\right)$ and times of maximum light. Although the SALT2 shape and color cuts are slightly asymmetric with respect to the mean of the SN Ia populations (Scolnic \& Kessler 2016), they are chosen primarily because they are the range within which the SALT2 model is valid. As measuring

\footnotetext{
17 See B14 for a detailed description of these data and their respective photometric systems.
} 
Table 1

SALT2-based Data Cuts

\begin{tabular}{lcccc}
\hline \hline & \multicolumn{3}{c}{ Number of SNe } & Comments \\
\cline { 2 - 4 } & PS1 Host- $z$ & PS1 SN- $z$ & Low- $z$ & \\
\hline Total candidates & 5235 & $\ldots$ & $\ldots$ & Likely host galaxy can be identified \\
Host Sep $R<5$ & 4461 & $\ldots$ & $\ldots$ & $\ldots$ \\
Good host redshifts & 3147 & $\ldots$ & $\ldots$ & SALT2 parameter fitting succeeds \\
Fit by SALT2 & 2534 & $\ldots$ & $\ldots$ & Separated from center or no long-term variability \\
Not an AGN & 2448 & 174 & 315 & SALT2 light-curve shape \\
$-3.0<x_{1}<3.0$ & 1938 & 168 & 296 & SALT2 light-curve color \\
$-0.3<c<0.3$ & 1523 & 160 & 258 & Uncertainty in time of max. light (rest-frame days) \\
$\sigma_{\text {peakMJD }}<2 \times(1+z)$ & 1490 & 159 & 254 & $x_{1}$ uncertainty \\
$\sigma_{x_{1}}<1$ & 1111 & 147 & 195 & $\chi^{2}$ and $N_{\text {dof }}$-based prob. from SALT2 fitter \\
Fit prob. $\geqslant 0.001$ & 1053 & 142 & 195 & Observed after maximum at 5 days $<t-t_{\mathrm{pk}}<45$ days \\
Obs. at $t-t_{\mathrm{pk}}>5$ days & 1031 & 137 & 195 & Milky Way reddening \\
$E(B-V)_{\mathrm{MW}}<0.15$ & 1031 & 137 &
\end{tabular}

Note. The host- $z$ column includes all PS1 SNe with the spectroscopic redshift of their host galaxy. The SN- $z$ column includes only spectroscopically classified PS1 SNe without spectroscopic host galaxy redshifts. The reasons for this distinction are due to selection biases and are discussed in Section 2.3.1.

cosmological parameters from $\mathrm{SNe}$ without spectroscopic classifications adds the potential for new biases to this work, we also strive for consistency with previous cosmological analyses whenever possible. For this reason, our cuts are nearly identical to those of B14, with two exceptions. The first is that we add a cut on the $\chi^{2}$ and degrees of freedom of the SALT2 light-curve fit (SALT2 fit probability $>0.001$ ) that was applied by Rest et al. (2014). This cut serves to remove CC SNe, as well as $\mathrm{SNe}$ Ia with poor light-curve fits. The second is that we require light curves to have at least one observation $>5$ days and $<45$ days after maximum, a cut that removes a total of $22 \mathrm{SNe}$. Without this cut, it is possible that some light-curve fits would have a multi-peaked probability distribution function for several SALT2 light-curve parameters (an issue raised by Dai \& Wang 2016). The cuts on $x_{1}$ uncertainty and time of maximum light uncertainty also serve to remove the biases that could arise from multi-peaked probability density functions (PDFs). We have not made a similar cut on the color uncertainty; although this uncertainty is often high, it should not bias the SN distances (and any bias would be removed by our bias-correction procedure; Section 2.3.2).

After fitting, we also remove a maximum of two light-curve epochs that lie $>3 \sigma$ from the best-fit SALT2 model. Of the lightcurve epochs between -15 days $<t_{\max }<45$ days, $1.3 \%$ are $3 \sigma$ outliers. We then rerun SALT2 with these data removed. The purpose of this procedure is to remove photometric data affected by unflagged image or subtraction defects without removing so many data points that CC SN light curves begin to resemble those of SNe Ia. Light-curve outlier removal increases the number of SNe passing the SALT2 fit probability cut by $\sim 10 \%$ (giving a slightly larger sample size than the one presented in J17) but does not noticeably increase the CC SN contamination.

The SALT2 cuts (Table 1) reduce the PS1 spectroscopically confirmed SN Ia sample by $\sim 30 \%$. They reduce the number of PS1 SNe Ia without spectroscopic classifications by $60 \%$, as these $\mathrm{SNe}$ have lower average $\mathrm{S} / \mathrm{N}^{18}$ and a lower fraction with SN Ia-like shapes and colors. Once shape, color, and $\sigma_{x_{1}}$ cuts have been applied, the time of maximum uncertainty cut and the fit probability cut remove similar fractions of $\mathrm{SNe}$ for both

\footnotetext{
18 These SNe more frequently fail the shape uncertainty cut. In PS1, SNe with $x_{1}$ uncertainty $<1$ have a mean $\mathrm{S} / \mathrm{N}$ at maximum light of 15.6. SNe with $x_{1}$ uncertainty $>1$ have a mean $\mathrm{S} / \mathrm{N}$ at maximum light of 8.3 .
}

photometric and spectroscopic samples. The Milky Way extinction cut removes no PS1 SNe, as the medium-deep fields were chosen to be in regions of the sky with low Milky Way $E$ $(B-V)$. The number of SNe remaining after each sample cut is shown in Table 1.

Table 1 also includes selection criteria that apply only to photometrically classified $\mathrm{SNe}$. These include a requirement that the host galaxy can be identified reliably (using the normalized separation between the $\mathrm{SN}$ and a galaxy center, $R$; Sullivan et al. 2006). We also remove potential active galactic nuclei (AGNs) by discarding SN candidates with both evidence for long-term variability and positions within 0 ." 5 of their host centers.

Once the light-curve parameters have been measured by using the SNANA fitting program to implement the SALT2 model, we use the Tripp estimator (Tripp 1998) to infer the SN distance modulus from these light-curve parameters:

$$
\mu=m_{B}-\mathcal{M}+\alpha \times x_{1}-\beta \times c+\Delta_{M}+\Delta_{B} .
$$

Here $x_{1}$ is the light-curve stretch parameter, $c$ is the light-curve color parameter, and $m_{B}$ is the log of the light-curve amplitude (approximately the peak SN magnitude in $B$ ). The distance to a given SN also depends on the global nuisance parameters $\alpha, \beta$, and $\mathcal{M} . \mathcal{M}-$ a combination of the absolute $\mathrm{SN}$ magnitude and the Hubble constant $-\alpha$, and $\beta$ is typically marginalized over when fitting to the cosmological parameters (e.g., B14; Conley et al. 2011). The parameter $\Delta_{M}$ is a correction based on the mass of the SN host galaxy, discussed in Section 2.2.1, and $\Delta_{B}$ is the distance bias correction, caused by SN selection effects. We use simulations to determine an initial $\Delta_{B}$ and apply it to the data (Section 2.3.2) before measuring $\alpha, \beta$, and $\Delta_{M}$. After $\alpha$ and $\beta$ have been measured, we redetermine $\Delta_{B}$ using the measured $\alpha$ and $\beta$ as input in the simulations. The simulated/ measured $\alpha$ and $\beta$ are given in Section 6 .

After light-curve fitting with the SALT2 model, even SNe Ia with low photometric uncertainties have a $\gtrsim 10 \%$ scatter in shape- and color-corrected magnitude. This is traditionally referred to as the intrinsic dispersion, $\sigma_{\text {int }}$ (Guy et al. 2007), which is defined as the global uncertainty that must be added in quadrature to the distance errors $\sigma_{\mu}$ of each SN such that the reduced $\chi^{2}$ of the Hubble residuals equals 1. This is not added to the uncertainty but kept as a free parameter in the 
cosmological parameter estimation. The SN Ia uncertainties also include redshift uncertainty and lensing uncertainty $\left(\sigma_{\text {lens }}=0.055 z\right.$; Jönsson et al. 2010).

\subsubsection{Host Galaxy Masses}

It has been shown that after shape and color correction, SNe Ia are $\sim 0.05-0.1$ mag brighter in high-mass host galaxies $\left(\log \left(M_{*} / M_{\odot}\right)>10\right)$ than in lower-mass host galaxies at the same redshifts $\left(\Delta_{M}\right.$; Kelly et al. 2010; Lampeitl et al. 2010; Sullivan et al. 2010). The $\Delta_{M}$ parameter has recently been measured at $>3 \sigma$ significance in photometrically classified SN samples, even though such samples (including PS1) have strong selection biases toward high-mass host galaxies (Campbell et al. 2016; Wolf et al. 2016; Uddin et al. 2017). Although the underlying physics behind the mass step are unclear, a simple step function appears to fit the SN data well (B14).

Computing $\Delta_{M}$ robustly requires measuring the host galaxy masses of every SN in a self-consistent way. We therefore measured host masses using the spectral energy distribution(SED-) fitting method of Pan et al. (2014) with PS1 and low- $z$ host galaxy photometry. For the low- $z$ sample, we use ugriz $B V R I J H K$ photometry from 2MASS (Skrutskie et al. 2006) and SDSS. For PS1, we use SExtractor (Bertin \& Arnouts 1996) to measure the photometry from PS1 templates. The PS1 templates are comprised of $\sim 3$ yr of co-added PS1 data, omitting only the year in which the SN Ia occurred.

The likely host of each $\mathrm{SN}$ is assumed to be the galaxy with the lowest $R$ parameter relative to the $\mathrm{SN}$ position, as discussed in J17. The $R$ parameter defines a separation between the SN and a galaxy center and is normalized by the size of the galaxy in the direction of the SN. ${ }^{19}$ If the nearest host has $R>5$ (i.e., the SN spectrum gives the only redshift), we assume the true host was undetected following Sullivan et al. (2006).

We use the low- $z$ and PS1 host galaxy photometry to estimate $M_{*}$ with the Z-PEG SED-fitting code (Le Borgne \& Rocca-Volmerange 2002), which in turn is based on spectral synthesis models from PEGASE . 2 (Fioc \& Rocca-Volmerange 1997). Galaxy SED templates correspond to spectral types SB, $\mathrm{Im}, \mathrm{Sd}, \mathrm{Sc}, \mathrm{Sbc}, \mathrm{Sa}, \mathrm{S} 0$, and E. We simultaneously marginalize over $E(B-V)$, which is allowed to vary from 0 to $0.2 \mathrm{mag}$. Uncertainties are determined from the range of model parameters that are able to fit the data with similar $\chi^{2}$ and are typically $\sim 0.1-0.3$ dex.

Undetected galaxies of spectroscopically classified $\mathrm{SNe}$ Ia are placed in the $\log \left(M_{*} / M_{\odot}\right)<10$ bin. At $z \gtrsim 0.5$, we cannot be sure that $\mathrm{SN}$ hosts have $\log \left(M_{*} / M_{\odot}\right)<10$, and we therefore add a systematic uncertainty of $0.07 \mathrm{mag}$ in quadrature to those distance uncertainties (similar to B14).

\subsection{SN Selection Bias}

\subsubsection{Simulating PS1 and Low-z SNe}

A magnitude-limited sample of $\mathrm{SNe}$ will have a distance bias, caused by SN selection effects, that can be determined from rigorous simulations of the survey (see, e.g., B14; Conley et al. 2011; Scolnic et al. 2014). We use the SNANA software (Kessler et al. 2010) to simulate SNe Ia based on the SALT2 model, with detection efficiencies, zero points, PSF sizes, sky

\footnotetext{
19 We predict that for $\sim 1 \%$ of SNe, this method will incorrectly determine the host galaxy, but in $\mathrm{J} 17$, we determined that this fraction of mismatches does not bias the cosmology.
}

noise, and other observables from the real PS1 and low- $z$ surveys. We generate the simulations using the values of $\alpha$ and $\beta$ measured from our data as input ( $\alpha=0.161$ and $\beta=3.060$; Section 5).

We use three survey simulations in this analysis: simulations of the set of PS1 SNe with redshifts from their host galaxies (the host- $z$ sample), the set of PS1 SNe Ia without host redshifts and with only redshifts from $\mathrm{SN}$ spectroscopy (the $\mathrm{SN}-z$ sample; these SNe have also been spectroscopically classified), and the compilation of low- $z$ SNe Ia. It is important that we use distinct simulations for $\mathrm{SNe}$ with and without host redshifts; because SN spectroscopy is only attempted for bright SNe $\left(r_{\mathrm{pk}} \lesssim 22\right)$, a lower magnitude limit than the PS1 survey detection limit comes into play for the $\mathrm{SN}-z$ sample. The $\mathrm{SN}-z$ sample includes only the portion of our data without host galaxy redshifts and thus is comprised almost entirely of $r_{\mathrm{pk}}<22$, spectroscopically classified $\mathrm{SNe}$ in faint hosts ( $r_{\text {host }} \gtrsim 22$ ). On the other hand, the host- $z$ sample is nearly an ideal, magnitude-limited SN sample, but it consists only of $\mathrm{SNe}$ in brighter ( $r \lesssim 22-23$ ) hosts. Even after shape and color correction, SN Ia luminosity is a function of the biased host galaxy properties in these samples, and we must correct for these biases using the $\Delta_{M}$ parameter (variants given in the systematic error analysis; Section 4). All PS1 simulations include photometric noise from the host galaxy, as discussed in J17. Simulations of the PS1 host- $z$ sample are presented in J17 (including CC SN contamination, which we discuss in detail in J17), while the $\mathrm{SN}-z$ and low $-z$ samples are presented in $\mathrm{S} 17$. The sizes of each of the three $\mathrm{SN}$ subsamples are given in Table 1.

The host- $z$ sample is also host galaxy magnitude-limited. Because SN shape and color correlate with host galaxy brightness (e.g., Childress et al. 2013), the SN shape and color distribution in the host- $z$ sample have a $z$ dependence that is difficult to model. Similarly, the $\mathrm{SN}-z$ sample consists of spectroscopically classified SNe for which host galaxy redshifts could not be measured, and therefore it will also have a biased, $z$-dependent host galaxy distribution. Because of this, we add one additional component to the host $z$ and $\mathrm{SN}-z$ simulations: we allow the means of the simulated SALT2 parameters $x_{1}$ and $c$ to evolve slightly with redshift to better match the data. We discuss the details and impact of this method in the Appendix and find that it changes the distance bias by up to $\sim 20$ mmag in the highest redshift bins but by less than $5 \mathrm{mmag}$ on average.

The low $-z$ surveys are exceptionally difficult to model due to their heterogeneous nature, multiple photometric systems and analysis pipelines, semi-arbitrary spectroscopic selection functions, and targeting of NGC galaxies. Furthermore, the cadence and depth of the search are often unknown. Because of this, we simulate both a "magnitude-limited" variant and a "volumelimited" variant of the low- $z$ survey. We treat the magnitudelimited variant as the baseline simulation for bias corrections. The volume-limited variant matches the observed data with a "host galaxy targeting" selection function-the fraction of hosts observed as a function of redshift-instead of a "spectroscopic follow-up" selection function (the fraction of SNe followed as a function of magnitude). Similarly to the PS1 simulations, we use redshift-dependent $x_{1}$ and $c$ distributions due to the redshift-dependent host galaxy properties $\left(x_{1} / c\right.$ and host properties are correlated; Childress et al. 2013). These simulations are discussed in more detail in S17. 


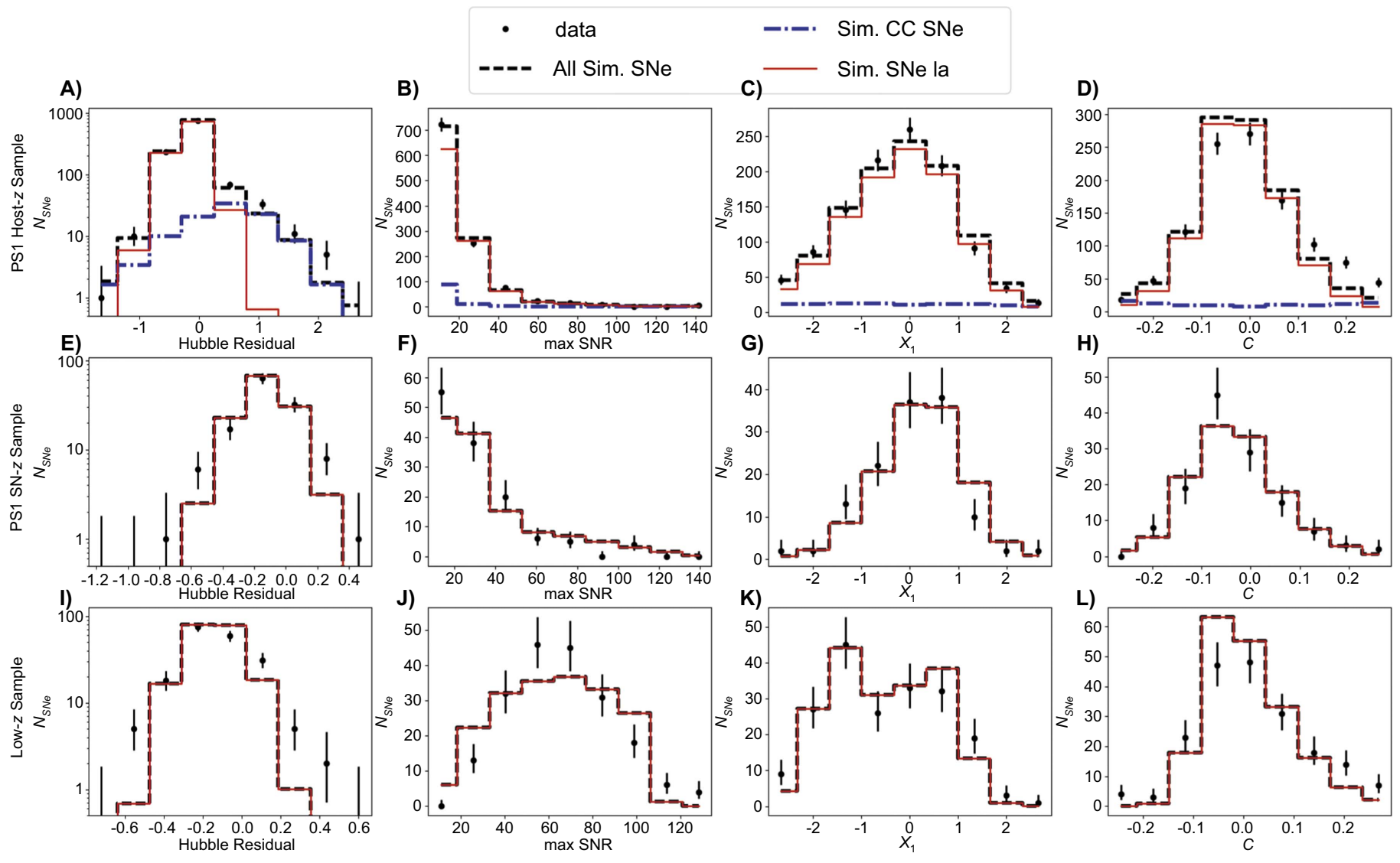

Figure 1. Simulations of the PS1 host galaxy redshift sample (host- $z$ sample), PS1 SN redshift sample (SN- $z$ sample), and low- $z$ SN sample compared to the real SNe used to measure cosmological parameters in this work. The PS1 host- $z$ sample consists of $\sim 9 \%$ CC SN contamination, the details of which are discussed in J17 (CC SN contamination is not relevant for distance bias correction).

For each survey, the simulations are compared to the data in Figures 1 and 2. The distributions of $x_{1}, c$, and their redshift dependences are consistent with the data, as is the distribution of SN S/Ns at maximum light. Discrepancies on the red tail of the $c$ distribution could be due to small inaccuracies in the CC SN simulations (J17). The biggest discrepancies between the simulations and data are found in the low- $z$ simulations due to the difficulty of modeling those searches and follow-up programs, as discussed above.

\subsubsection{Using Simulations to Correct for Selection Bias}

Due to their intrinsic dispersion, SNe Ia discovered in magnitude-limited surveys appear increasingly luminous at greater distance-even after shape and color correction. Even the low- $z$ SN Ia surveys used here may be biased toward preferentially selecting brighter SNe Ia for spectroscopic follow-up (see B14, their Figure 5). The bias in distance is given by the SNANA simulations discussed above and is defined by (Mosher et al. 2014)

$$
\Delta_{B}(z)=\left\langle\mu_{\text {fit }}-\mu_{\text {sim }}\right\rangle_{z} .
$$

For low- $z$ surveys, the bias can be up to $\sim 0.035 \mathrm{mag}$ $(z>0.05)$, while PS1 has distance biases of nearly $0.1 \mathrm{mag}$ at $z>0.5$.

Uncertainty in the intrinsic dispersion model is the dominant uncertainty in the bias corrections. The uncertainty is encapsulated by two primary scatter models that are both consistent with the data. First, the G10 SALT2 model assumes
A)
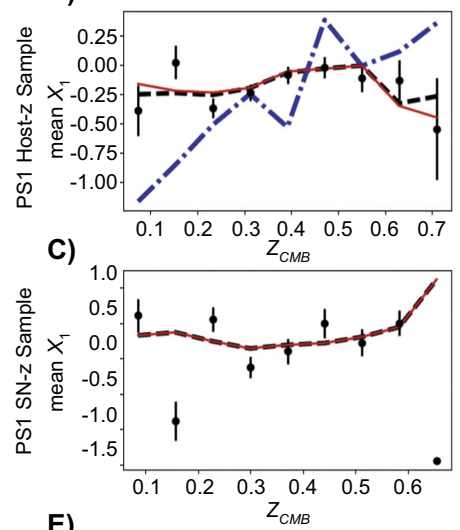

E)

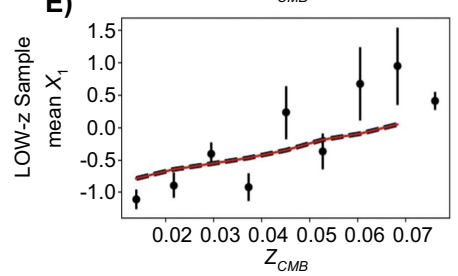

Figure 2. Similar to Figure 1 (see Figure 1 legend) but showing the dependence of $x_{1}$ and $c$ on redshift for each survey.

that $70 \%$ of the $\sim 0.1 \mathrm{mag}$ intrinsic dispersion in derived SN Ia distances is uncorrelated with the shape or color of the SN (achromatic dispersion). An alternative model is that of Chotard et al. (2011, hereafter C11). C11 found an equally 


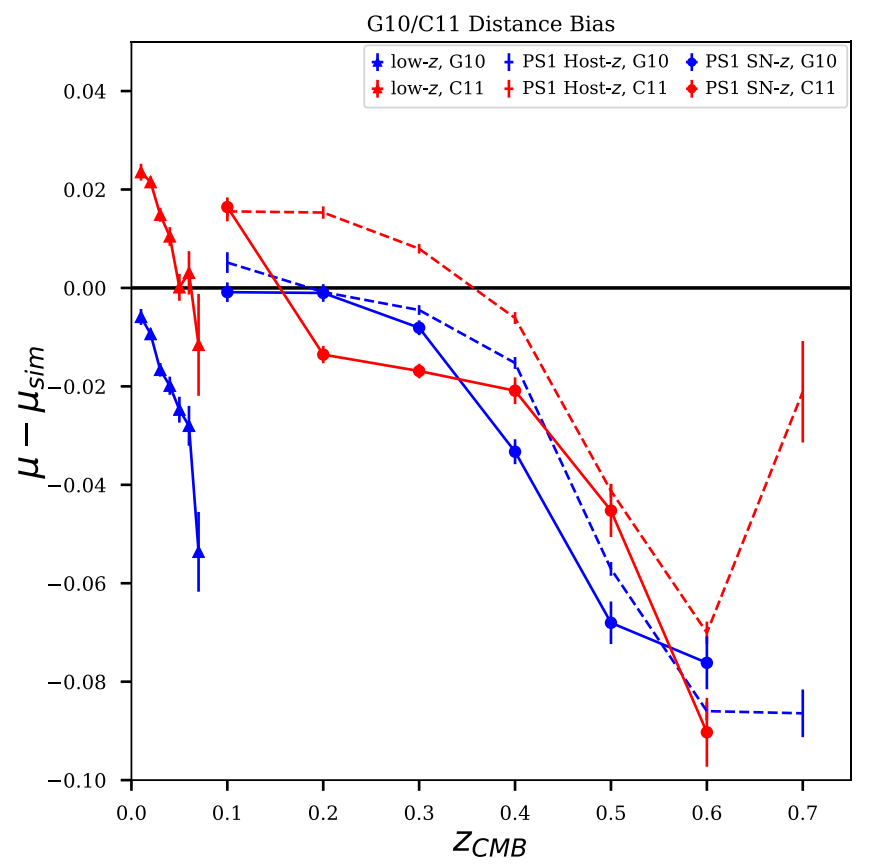

Figure 3. Difference in $\mathrm{SN}$ Ia distance bias for the G10 and $\mathrm{C} 11$ scatter models. Low $z$ SNe have a bias of up to 0.035 mag, while PS1 SNe have a bias of up to $\sim 0.1 \mathrm{mag}$ at the highest survey redshifts. At the highest survey redshifts, where few SNe Ia can be discovered, the $\mathrm{C} 11$ model predicts a drastically different bias from that of the G10 model due to selection and measurement biases in the SALT2 $c$ parameter.

good fit to SN data by assuming $75 \%$ of $\mathrm{SN}$ dispersion can be attributed to chromatic variation.

The host $z$ and $\mathrm{SN}-z$ biases are very similar, which is surprising given that $\mathrm{SNe}$ in the $\mathrm{SN}-z$ sample are much brighter, on average, than those in the host $z$ sample. The reason is that the lower average $\mathrm{S} / \mathrm{N}$ of the host- $z$ sample exacerbates a bias caused by the $x_{1}$ uncertainty cut. At a given $\mathrm{S} / \mathrm{N}, \mathrm{SNe}$ with narrower (measured) light-curve shapes are given lower $x_{1}$ uncertainties by SALT2. This introduces a nonintuitive bias in the case where many $x_{1}$ uncertainties are near the cutoff point (for inclusion in our sample) of $\sigma_{x_{1}}=1$. As discussed in J17 (Figure 8), a $\sigma_{x_{1}}<1$ sample cut biases the recovered values of $x_{1}$ by up to $\alpha\left(x_{1}-x_{1, \text { sim }}\right)=-0.1$ at high$z$. The size of this bias is similar to the size of the $m_{B}$ bias of spectroscopically confirmed SNe Ia $(\sim 0.05 \mathrm{mag}$ at $z \sim 0.5)$.

The SALT2 nuisance parameter $\beta$ is $25 \%$ higher in the $\mathrm{C} 11$ model than in the G10 model (Scolnic \& Kessler 2016), and these two models can give very different predictions for the distance bias as a function of redshift (Figure 3). Due to the chromatic nature of the $\mathrm{C} 11$ dispersion, the $\mathrm{C} 11$ bias is a strong function of the (z-dependent) $\mathrm{SN} c$ distribution in a given survey. This is especially apparent when examining the difference between the $\mathrm{G} 10 / \mathrm{C} 11$ biases for the different samples. Low $-z$ and photometrically classified $\mathrm{SNe}$ have a median $c$ between -0.01 and 0.01 , giving an average $\beta^{C 11} c-\beta^{G 10} c=0.015 \mathrm{mag}$ for low $-z$ and $0.003 \mathrm{mag}$ for PS1 photometrically classified SNe. In contrast, PS1 spectroscopically confirmed SNe Ia have a median $c$ of -0.04 , giving an average difference of $\beta^{C 11} c-\beta^{G 10} c=-0.028 \mathrm{mag}$ in distance. Unfortunately, there are not enough spectroscopically classified SNe Ia to distinguish between the G10/C11 scatter models in our data, and the differences between these two model predictions will contribute to our systematic error budget.

\subsection{Photometric Classification}

In the previous sections, we made SALT2-based cuts and distance bias corrections to our data without requiring any knowledge as to which of the $\mathrm{SNe}$ in the photometrically classified sample were SNe Ia. We now use PSNID (Sako et al. 2014) to classify each SN in this sample as Type Ia, Ib/c, or II based on its light curve. PSNID matches observed SN light curves to simulated SN Ia and CC SN light curves. The comparison of data to templates gives a $\chi^{2}$ and prior-based probability that a given $\mathrm{SN}$ is Type Ia. We use the version of PSNID that has been implemented in SNANA. ${ }^{20}$ For SNe Ia, we use the SALT2 model as the PSNID SN Ia template, and for CC SNe, PSNID marginalizes over 51 CC SN templates when classifying SNe. We include a grid of host galaxy reddening values for each template (because templates have not been corrected for host galaxy reddening, we allow just $0<A_{V}<1$ of additional reddening).

Although PSNID classifications will be used for the baseline version of our cosmological analysis (Section 3), we also use three alternate classification methods. These include two lightcurve-based methods, nearest neighbor (NN; Sako et al. 2014; Kessler \& Scolnic 2017) and Fitprob. The NN classifier uses the proximity of SN light-curve parameters to the SALT2 $x_{1}, c$, and redshift of simulated CC and Ia SNe to determine the likely SN type. Fitprob is the fit probability from the SALT2 lightcurve fit multiplied by a redshift-dependent SN type prior. This prior is based on simulations, which give the expected fractions of CC SNe and SNe Ia at each redshift (J17, Appendix B). One additional method, GalSNID (Foley \& Mandel 2013; J17), takes advantage of the paucity of CC SNe in low star formation environments to estimate the $\mathrm{SN}$ type probability from only host galaxy properties. Fitprob and GalSNID are less accurate classifiers (J17) but are also less subject to the uncertainties in CC SN simulations. In J17, we suggest that uncertainties in the shape of CC SN luminosity functions and the dearth of CC SN templates for several subtypes necessitate the use of methods that are less reliant on simulations.

Figure 4 shows the classification probabilities for three PS1 SNe with ambiguous types. For SN 570024 (top panel), two of three light-curve-based classification methods agree that this $\mathrm{SN}$ is most likely a CC SN due to its poor SALT2 model fit in the $z$-band. GalSNID, however, finds that this is most likely a bona fide Ia due to the lack of strong star formation indicators in its host galaxy spectrum. For SN 500025 (middle panel), PSNID and Fitprob agree that the SN is of Type Ia due to the low $\chi^{2}$ of its light-curve fit. However, the NN classifier finds it most likely to be a CC SN due to its red SALT2 color. For SN 550152 (bottom panel), the shapes/colors are consistent with a $\mathrm{SN}$ Ia, but the light-curve fit $\chi^{2}$ is too high to definitively prefer a SN Ia. This diversity in classification methodologies and outcomes will help our systematic uncertainty budget to account for the possibility of cosmological bias due to mistyped SNe.

Figure 5 illustrates the classification probabilities. We show the PS1 Hubble residual histograms for likely SNe Ia and $\mathrm{CC} \mathrm{SNe}$ without spectroscopic classifications as determined by each of the four classification methods considered in this work.

\footnotetext{
${ }^{20}$ Version $10.52 \mathrm{~g}$.
} 

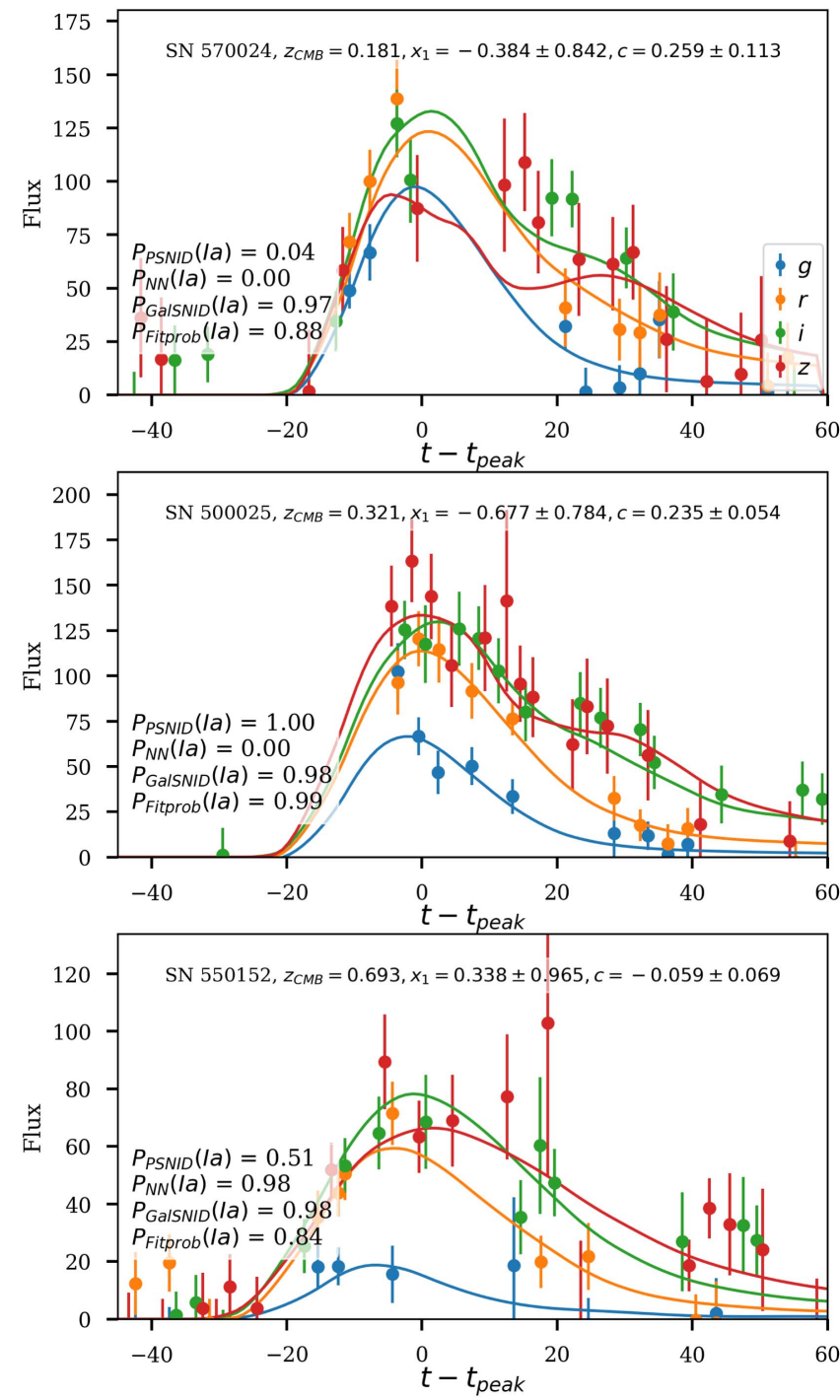

Figure 4. Three PS1 light curves with ambiguous classifications included in our sample. The curves show their best-fit SALT2 light-curve fits.

As a diagnostic, if we assume all $\mathrm{SNe}$ with Hubble residual $>1$ are $\mathrm{CC} \mathrm{SNe}$, we find that PSNID classifies $80 \%$ of these CC SNe correctly, while NN classifies $60 \%$ correctly. Fitprob and GalSNID classify $70 \%$ and $20 \%$ correctly, respectively. We note that PSNID is unable to classify all SNe, rejecting 13 as too noisy or uncertain for classification. We revisit the effect of different classifiers on our results in Section 5.

\section{Cosmological Parameter Estimation Methodology}

In the previous section, we measured the SALT2 light-curve parameters, host galaxy masses, SN type probabilities, and bias corrections that will be used to generate distances from PS1 and low- $z$ SNe Ia. For each SN in our final sample, these parameters are given in Table 2. Host galaxy coordinates and redshift information are given in Table 3. Light curves and host galaxy spectra are available at https://doi.org.10.17909/T95Q4X. From this point forward, we use all PS1 and low- $z$ data combined - data with and without spectroscopic classifications - to obtain the best possible measurements of cosmological parameters. We use the PSNID classifications to generate our baseline, statistics-only cosmological parameter measurements
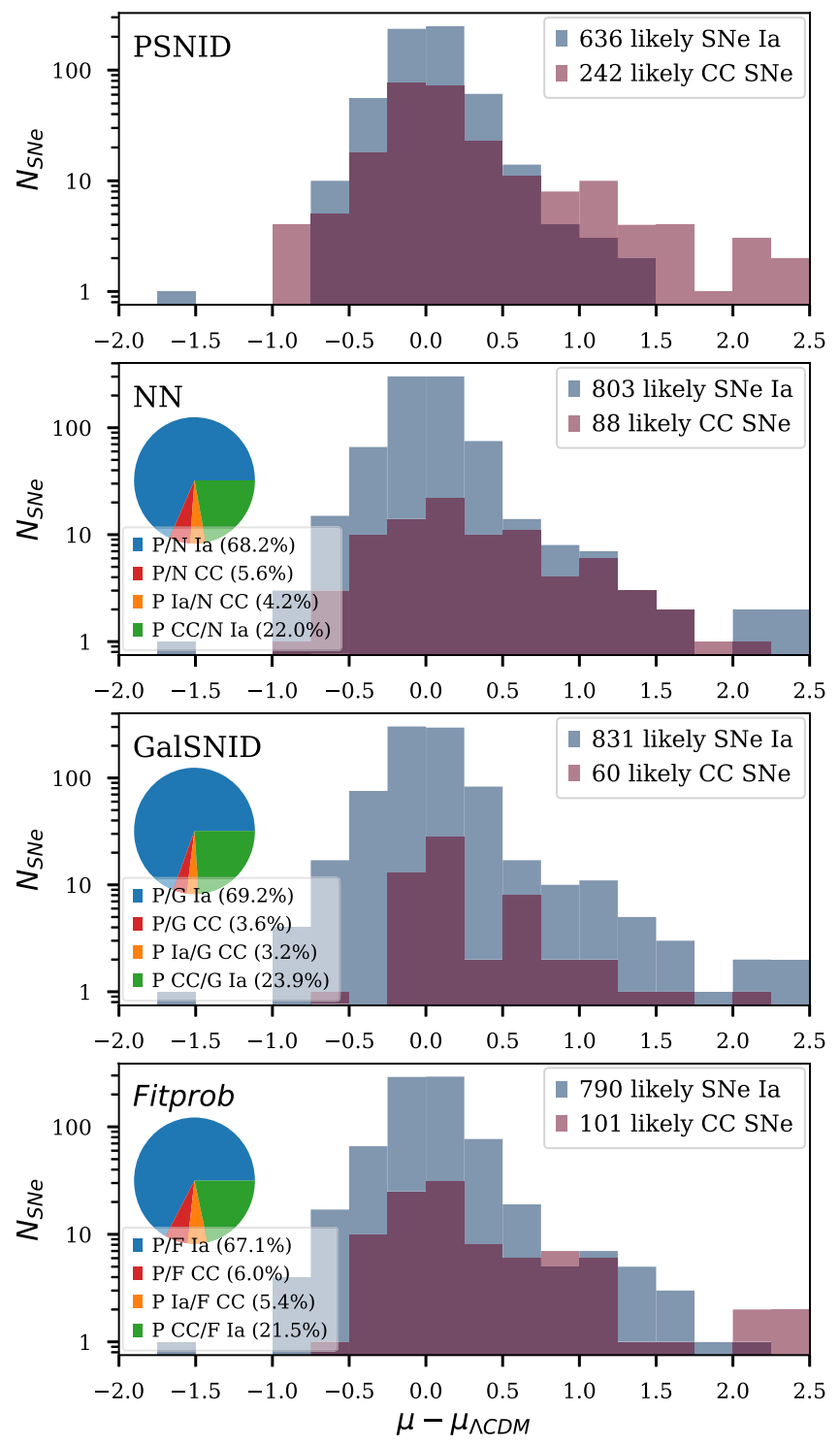

Figure 5. For SNe without spectroscopic classifications, log-scaled histograms of Hubble residuals for likely PS1 SNe Ia (P(Ia) $>0.5$; blue) and likely PS1 $\mathrm{CC} \mathrm{SNe}(\mathrm{P}(\mathrm{Ia})<0.5$; red $)$ from each classifier considered in this work. Fitprob classifies the most real SNe Ia as CC SNe, while GalSNID likely classifies the most real CC SNe as SNe Ia. In spite of large classification differences, the SN Ia distances given by different classifiers will be shown to be consistent with each other and with the spectroscopically confirmed PS1 sample (Section 5). The pie charts show the level of agreement/disagreement between each classifier and PSNID, where P, N, G, and F indicate PSNID, NN, GalSNID, and Fitprob classifications. In these pie charts, we label SNe with $\mathrm{P}(\mathrm{Ia})>0.5$ as Ia and $\mathrm{SNe}$ with $\mathrm{P}(\mathrm{Ia})<0.5$ as $\mathrm{CC}$.

and incorporate the other classification methods into our systematic uncertainty budget. To reduce CC SN contamination, we apply one additional cut on a classifier-by-classifier basis before estimating cosmological parameters: we remove $\mathrm{SNe}$ with $\mathrm{P}(\mathrm{Ia})<0.5$. Therefore, 1109 likely SNe Ia will be used in our baseline cosmological analysis, and between 1263 and 1304 $\mathrm{SNe}$ will be used for the alternate classification methods.

For some readers, the most interesting question might be whether future cosmological analyses, such as those of DES or LSST, can robustly measure $w$ without a spectroscopically classified SN sample as part of the data. We explore this question in Section 8. 
Table 2

PS1 Coordinates and Light-curve Parameters

\begin{tabular}{|c|c|c|c|c|c|c|c|c|c|c|}
\hline $\mathrm{SN}$ & $\alpha$ & $\delta$ & $z_{\mathrm{CMB}}^{\mathrm{SN}}$ & 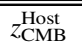 & $\begin{array}{l}t_{\text {peak }} \\
\end{array}$ & $\overline{x_{1}}$ & $\overline{c c}$ & $m_{B}$ & $P_{\text {PSNID }}(\mathrm{Ia})^{\mathrm{a}}$ & 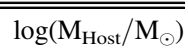 \\
\hline 010196 & $12: 16: 49.602$ & $46: 14: 06.33$ & $\ldots$ & 0.369 & $55246.40(0.20)$ & $-1.457(0.876)$ & $0.228(0.075)$ & $22.970(0.076)$ & $\ldots$ & $11.192(0.155)$ \\
\hline 010203 & 08:40:02.784 & $43: 26: 32.85$ & 0.088 & 0.087 & $55230.60(0.10)$ & $1.234(0.163)$ & $-0.061(0.028)$ & $18.135(0.046)$ & $\ldots$ & $10.384(0.009)$ \\
\hline 010204 & $08: 41: 36.065$ & $43: 24: 02.18$ & 0.477 & 0.477 & $55241.10(0.40)$ & $-1.727(0.839)$ & $-0.032(0.048)$ & $22.841(0.015)$ & 0.9957 & $10.519(0.315)$ \\
\hline 010218 & 09:54:32.47 & $01: 56: 37.53$ & $\ldots$ & 0.577 & $55248.70(0.50)$ & $0.509(0.768)$ & $-0.098(0.056)$ & $23.212(-0.002)$ & 0.8756 & $9.737(0.151)$ \\
\hline 010222 & $12: 16: 56.796$ & $47: 17: 21.68$ & $\ldots$ & 0.408 & $55241.30(0.40)$ & $0.139(0.523)$ & $0.028(0.036)$ & $22.386(0.024)$ & 0.9998 & $10.164(0.301)$ \\
\hline 010230 & $12: 21: 10.815$ & $47: 48: 13.43$ & $\ldots$ & 0.303 & $55246.30(0.40)$ & $-0.659(0.362)$ & $-0.042(0.035)$ & $21.768(0.043)$ & 1.0000 & $10.395(0.157)$ \\
\hline 010430 & $08: 45: 12.962$ & $43: 52: 36.68$ & $\ldots$ & 0.327 & $55258.20(0.50)$ & $-1.447(0.428)$ & $-0.029(0.068)$ & $21.886(0.090)$ & 0.9622 & $12.070(0.194)$ \\
\hline 020026 & $12: 15: 12.803$ & $46: 02: 40.40$ & $\ldots$ & 0.321 & $55276.60(0.40)$ & $0.314(0.325)$ & $-0.127(0.031)$ & $21.485(0.036)$ & 1.0000 & $10.824(0.041)$ \\
\hline 020033 & 12:17:03.99 & $46: 04: 22.76$ & $\ldots$ & 0.530 & $55268.50(1.50)$ & $-0.888(0.962)$ & $-0.065(0.074)$ & $22.858(0.041)$ & $\ldots$ & $11.737(0.013)$ \\
\hline 020034 & 12:18:12.126 & $46: 05: 10.35$ & $\cdots$ & 0.199 & $55272.10(0.30)$ & $-1.165(0.431)$ & $0.085(0.034)$ & $20.828(0.042)$ & $\ldots$ & $11.037(0.049)$ \\
\hline 020047 & 09:56:56.438 & $01: 36: 49.80$ & $\cdots$ & 0.266 & $55260.20(0.80)$ & $-0.952(0.884)$ & $0.111(0.151)$ & $22.832(0.224)$ & 0.9850 & $10.528(0.150)$ \\
\hline 020075 & $14: 18: 54.904$ & 53:59:57.08 & 0.156 & 0.157 & $55286.80(0.40)$ & $2.350(0.465)$ & $0.207(0.053)$ & $21.395(0.085)$ & $\cdots$ & $8.795(0.217)$ \\
\hline 020104 & 09:58:36.773 & 02:17:37.70 & $\cdots$ & 0.306 & $55277.10(0.60)$ & $0.701(0.533)$ & $-0.080(0.026)$ & $21.507(0.040)$ & 0.9314 & $10.905(0.257)$ \\
\hline 020123 & $12: 22: 25.600$ & 48:02:28.66 & $\cdots$ & 0.498 & $55265.30(1.40)$ & $-0.668(0.967)$ & $-0.018(0.087)$ & $22.856(0.062)$ & 0.9915 & $10.532(0.382)$ \\
\hline 020148 & $10: 38: 21.978$ & $57: 23: 24.30$ & 0.102 & 0.102 & $55259.70(0.20)$ & $-1.103(0.241)$ & $-0.023(0.036)$ & $18.980(0.059)$ & $\cdots$ & $11.306(0.065)$ \\
\hline 020194 & 09:58:34.811 & $00: 49: 52.10$ & $\ldots$ & 0.246 & $55277.90(0.50)$ & $-1.012(0.734)$ & $0.079(0.058)$ & $21.420(0.069)$ & $\ldots$ & $10.965(0.133)$ \\
\hline 020198 & $10: 46: 52.536$ & $57: 07: 45.51$ & 0.361 & 0.361 & $55286.20(0.70)$ & $-0.805(0.891)$ & $-0.083(0.081)$ & $22.284(0.119)$ & 0.5443 & $10.034(0.033)$ \\
\hline 020200 & $14: 12: 11.636$ & $53: 27: 47.48$ & 0.116 & 0.116 & $55291.10(0.20)$ & $0.005(0.166)$ & $0.110(0.037)$ & $20.513(0.062)$ & $\ldots$ & $10.400(0.186)$ \\
\hline 030005 & $12: 25: 23.414$ & $47: 29: 11.16$ & $\ldots$ & 0.420 & $55284.70(0.60)$ & $-1.345(0.617)$ & $-0.002(0.076)$ & $22.315(0.083)$ & 0.9810 & $11.270(0.018)$ \\
\hline 030007 & 14:09:23.651 & $53: 37: 06.96$ & $\ldots$ & 0.260 & $55293.80(0.40)$ & $-0.669(0.337)$ & $0.067(0.050)$ & $21.516(0.076)$ & 1.0000 & $10.030(0.279)$ \\
\hline 030068 & 12:14:39.906 & $48: 05: 21.86$ & $\ldots$ & 0.296 & $55294.70(0.30)$ & $-0.310(0.789)$ & $-0.012(0.076)$ & $22.474(0.096)$ & 0.9989 & $11.457(0.102)$ \\
\hline 030216 & $14: 14: 56.573$ & $54: 12: 41.36$ & $\ldots$ & 0.198 & $55304.10(0.90)$ & $-2.157(0.532)$ & $0.010(0.041)$ & $21.768(0.069)$ & $\ldots$ & $10.799(0.094)$ \\
\hline 030245 & 12:26:08.645 & $46: 30: 52.82$ & $\cdots$ & 0.581 & $55290.20(0.80)$ & $-0.633(0.686)$ & $-0.188(0.076)$ & $22.748(0.017)$ & 1.0000 & $10.450(0.451)$ \\
\hline 030252 & $12: 17: 28.972$ & $48: 05: 38.05$ & $\cdots$ & 0.326 & $55319.10(0.60)$ & $0.746(0.552)$ & $0.094(0.055)$ & $22.240(0.091)$ & 1.0000 & $8.784(0.193)$ \\
\hline 030263 & 12:20:47.701 & 48:10:01.13 & $\ldots$ & 0.299 & $55312.00(0.20)$ & $0.717(0.275)$ & $-0.049(0.030)$ & $21.168(0.047)$ & 1.0000 & $10.116(0.310)$ \\
\hline 040121 & 10:39:04.003 & $58: 35: 25.74$ & $\ldots$ & 0.322 & $55309.50(0.40)$ & $0.561(0.368)$ & $-0.030(0.038)$ & $21.527(0.049)$ & 1.0000 & $9.409(0.281)$ \\
\hline 040139 & 14:17:09.899 & 53:05:11.39 & $\cdots$ & 0.267 & $55324.30(0.90)$ & $-0.211(0.306)$ & $0.146(0.036)$ & $21.599(0.057)$ & 1.0000 & $10.224(0.281)$ \\
\hline 040147 & $14: 15: 40.447$ & $54: 13: 43.85$ & $\cdots$ & 0.244 & $55317.40(0.40)$ & $-0.927(0.293)$ & $0.100(0.043)$ & $21.316(0.073)$ & $\ldots$ & $10.459(0.148)$ \\
\hline 040151 & $12: 21: 49.674$ & $46: 27: 04.69$ & $\ldots$ & 0.256 & $55326.30(0.20)$ & $-1.576(0.326)$ & $0.014(0.032)$ & $21.473(0.043)$ & 1.0000 & $11.207(0.015)$ \\
\hline 040163 & 12:22:04.649 & $47: 00: 36.58$ & $\ldots$ & 0.416 & $55321.30(0.20)$ & $-0.248(0.470)$ & $-0.070(0.051)$ & $22.302(0.046)$ & 1.0000 & $9.822(0.632)$ \\
\hline 040168 & $12: 27: 10.791$ & $47: 11: 23.08$ & $\ldots$ & 0.206 & $55325.00(0.20)$ & $-0.342(0.541)$ & $0.106(0.047)$ & $20.953(0.053)$ & $\ldots$ & $10.898(0.084)$ \\
\hline 040169 & $10: 49: 29.313$ & $58: 45: 59.05$ & $\ldots$ & 0.421 & $55332.60(0.30)$ & $0.208(0.937)$ & $-0.009(0.064)$ & $22.716(0.059)$ & 0.6477 & $10.609(0.241)$ \\
\hline 040170 & $12: 14: 21.336$ & $47: 50: 35.25$ & $\cdots$ & 0.190 & $55330.40(0.30)$ & $-0.935(0.187)$ & $-0.009(0.028)$ & $20.484(0.039)$ & $\cdots$ & $11.449(0.046)$ \\
\hline 040176 & $12: 20: 58.358$ & 45:56:04.95 & $\ldots$ & 0.348 & $55327.30(1.70)$ & $1.351(0.778)$ & $0.025(0.042)$ & $21.788(0.047)$ & 1.0000 & $10.992(0.053)$ \\
\hline 040313 & $12: 16: 25.19$ & $48: 21: 56.92$ & $\cdots$ & 0.266 & $55335.80(0.60)$ & $-0.394(0.727)$ & $0.235(0.056)$ & $22.598(0.083)$ & 0.9996 & $9.791(0.105)$ \\
\hline 040316 & 14:11:23.481 & $52: 26: 04.60$ & $\cdots$ & 0.443 & $55324.00(0.20)$ & $0.286(0.782)$ & $0.216(0.075)$ & $22.913(0.054)$ & 0.6527 & $10.464(0.140)$ \\
\hline 040318 & 14:17:19.799 & $53: 06: 45.28$ & $\ldots$ & 0.300 & $55334.40(0.20)$ & $0.109(0.617)$ & $-0.013(0.050)$ & $22.065(0.062)$ & 0.6791 & $10.588(0.272)$ \\
\hline 040343 & 10:39:09.733 & $58: 40: 39.35$ & $\cdots$ & 0.343 & $55334.00(0.70)$ & $-0.019(0.645)$ & $0.107(0.055)$ & $22.250(0.055)$ & 0.9789 & $10.898(0.022)$ \\
\hline 040377 & $10: 40: 51.886$ & $58: 52: 53.50$ & $\cdots$ & 0.352 & $55302.40(1.10)$ & $1.248(0.629)$ & $0.125(0.067)$ & $22.158(0.071)$ & 0.9959 & $9.750(0.467)$ \\
\hline 040434 & $12: 21: 24.625$ & $45: 53: 41.62$ & $\cdots$ & 0.654 & $55315.60(0.90)$ & $-1.624(0.838)$ & $-0.059(0.089)$ & $23.100(0.048)$ & $\cdots$ & $10.861(0.018)$ \\
\hline 040473 & $10: 58: 22.122$ & $58: 28: 59.27$ & $\ldots$ & 0.161 & $55302.60(1.40)$ & $0.056(0.571)$ & $-0.007(0.130)$ & $19.982(0.146)$ & $\ldots$ & $10.964(0.029)$ \\
\hline 040477 & $16: 20: 34.012$ & $54: 48: 24.17$ & $\ldots$ & 0.346 & $55332.00(0.60)$ & $0.696(0.418)$ & $-0.023(0.032)$ & $21.532(0.031)$ & 1.0000 & $11.003(0.037)$ \\
\hline
\end{tabular}

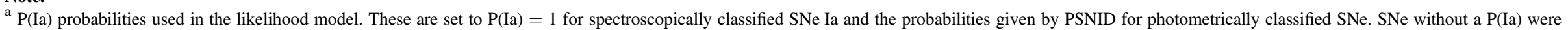
unable to be classified by PSNID

(This table is available in its entirety in machine-readable form.) 
Table 3

PS1 Host Galaxies

\begin{tabular}{|c|c|c|c|c|c|c|}
\hline $\mathrm{SN}$ & Host $\alpha$ & Host $\delta$ & $z_{\mathrm{CMB}}^{\text {Host }}$ & Normalized Sep. $^{\text {a }}$ & $\mathrm{T} \& \mathrm{D} R^{\mathrm{b}}$ & $z_{\text {source }}$ \\
\hline 010196 & $12: 16: 49.577$ & $46: 14: 06.27$ & 0.369 & 0.624 & 7.340 & MMT/Hecto \\
\hline 010203 & 08:40:02.725 & $43: 26: 33.14$ & 0.087 & 1.044 & 23.880 & MMT/Hecto \\
\hline 010204 & $08: 41: 36.025$ & $43: 24: 02.56$ & 0.477 & 1.741 & 8.080 & MMT/Hecto \\
\hline 010218 & 09:54:32.455 & 01:56:38.21 & 0.577 & 2.468 & 4.280 & MMT/Hecto \\
\hline 010222 & 12:16:56.817 & 47:17:22.39 & 0.408 & 1.777 & 7.280 & MMT/Hecto \\
\hline 010230 & $12: 21: 10.792$ & 47:48:13.10 & 0.303 & 1.413 & 9.420 & MMT/Hecto \\
\hline 010430 & $08: 45: 12.976$ & $43: 52: 38.13$ & 0.327 & 1.440 & $\ldots$ & SDSS \\
\hline 020026 & $12: 15: 12.784$ & $46: 02: 41.30$ & 0.321 & 1.287 & 5.040 & MMT/Hecto \\
\hline 020033 & 12:17:04.116 & $46: 04: 20.33$ & 0.530 & 2.815 & 8.830 & MMT/Hecto \\
\hline 020034 & 12:18:12.000 & 46:05:10.87 & 0.199 & 1.525 & 7.380 & MMT/Hecto \\
\hline 020047 & 09:56:56.503 & 01:36:49.99 & 0.266 & 1.103 & 4.200 & MMT/Hecto \\
\hline 020075 & $14: 18: 54.883$ & 53:59:57.18 & 0.157 & 1.780 & 13.710 & MMT/Hecto \\
\hline 020104 & 09:58:36.813 & $02: 17: 37.53$ & 0.306 & 0.119 & 14.020 & MMT/Hecto \\
\hline 020123 & $12: 22: 25.692$ & 48:02:29.95 & 0.498 & 2.874 & 4.190 & MMT/Hecto \\
\hline 020148 & $10: 38: 21.820$ & $57: 23: 23.82$ & 0.102 & 0.683 & $\cdots$ & SDSS \\
\hline 020194 & 09:58:34.812 & 00:49:51.78 & 0.246 & 0.393 & 8.830 & MMT/Hecto \\
\hline 020198 & $10: 46: 52.540$ & 57:07:45.40 & 0.361 & 0.448 & 12.460 & MMT/Hecto \\
\hline 020200 & $14: 12: 11.216$ & 53:27:50.95 & 0.116 & 3.171 & 18.190 & MMT/Hecto \\
\hline 030005 & $12: 25: 23.424$ & 47:29:10.85 & 0.420 & 0.523 & 7.480 & MMT/Hecto \\
\hline 030007 & $14: 09: 23.635$ & 53:37:07.06 & 0.260 & 1.275 & 11.920 & MMT/Hecto \\
\hline 030068 & $12: 14: 39.72$ & 48:05:22.10 & 0.296 & 2.365 & 8.320 & MMT/Hecto \\
\hline 030216 & $14: 14: 56.627$ & $54: 12: 43.21$ & 0.198 & 1.582 & 8.360 & MMT/Hecto \\
\hline 030245 & 12:26:08.666 & 46:30:52.84 & 0.581 & 0.785 & 4.610 & WIYN/Hydra \\
\hline 030252 & $12: 17: 28.923$ & 48:05:38.05 & 0.326 & 2.510 & 6.510 & WIYN/Hydra \\
\hline 030263 & $12: 20: 47.774$ & 48:10:00.74 & 0.299 & 0.370 & 4.970 & MMT/Hecto \\
\hline 040121 & 10:39:03.921 & $58: 35: 27.14$ & 0.322 & 1.853 & 4.550 & MMT/Hecto \\
\hline 040139 & 14:17:09.885 & 53:05:11.20 & 0.267 & 0.864 & 23.550 & MMT/Hecto \\
\hline 040147 & $14: 15: 40.454$ & $54: 13: 43.82$ & 0.244 & 0.194 & 8.630 & MMT/Hecto \\
\hline 040151 & $12: 21: 49.768$ & $46: 27: 06.13$ & 0.256 & 2.506 & 16.440 & MMT/Hecto \\
\hline 040163 & $12: 22: 04.663$ & 47:00:37.89 & 0.416 & 2.993 & 5.520 & MMT/Hecto \\
\hline 040168 & $12: 27: 10.792$ & $47: 11: 22.92$ & 0.206 & 0.632 & 16.550 & MMT/Hecto \\
\hline 040169 & $10: 49: 29.349$ & 58:45:59.01 & 0.421 & 0.788 & 8.350 & MMT/Hecto \\
\hline 040170 & $12: 14: 21.256$ & $47: 50: 39.52$ & 0.190 & 3.676 & 21.880 & MMT/Hecto \\
\hline 040176 & $12: 20: 58.375$ & 45:56:04.84 & 0.348 & 0.455 & 11.590 & MMT/Hecto \\
\hline 040313 & 12:16:25.161 & 48:21:56.79 & 0.266 & 1.481 & 7.600 & MMT/Hecto \\
\hline 040316 & $14: 11: 23.433$ & $52: 26: 04.15$ & 0.443 & 1.471 & 11.610 & MMT/Hecto \\
\hline 040318 & $14: 17: 19.807$ & 53:06:45.00 & 0.300 & 0.646 & 12.360 & MMT/Hecto \\
\hline 040343 & 10:39:09.748 & $58: 40: 39.15$ & 0.343 & 0.628 & 5.380 & MMT/Hecto \\
\hline 040377 & 10:40:51.832 & 58:52:53.22 & 0.352 & 0.643 & 15.250 & MMT/Hecto \\
\hline 040434 & $12: 21: 24.595$ & 45:53:41.62 & 0.654 & 1.559 & 4.260 & MMT/Hecto \\
\hline 040473 & $10: 58: 22.401$ & 58:29:00.95 & 0.161 & 2.872 & 17.760 & MMT/Hecto \\
\hline 040477 & $16: 20: 33.996$ & 54:48:24.00 & 0.346 & 0.888 & 11.110 & MMT/Hecto \\
\hline
\end{tabular}

Notes.

${ }^{a}$ Separation of the SN from the center of its host galaxy, normalized by the size and orientation of the host (the $R$ parameter; Sullivan et al. 2006). The isophotal radius of a galaxy corresponds to $R \simeq 3$.

${ }^{\mathrm{b}}$ The Tonry \& Davis (1979) cross-correlation parameter, computed by comparing the host galaxy spectrum to a template spectrum to determine the host redshift. Redshifts with $R>4$ are treated as reliable in this work, though $1.4 \%$ of all redshifts are expected to be spurious, as discussed in J17.

(This table is available in its entirety in machine-readable form.)

With these data, we measure cosmological parameters from 1169 PS1 SNe and 195 low- $z$ SNe Ia in two steps: (1) marginalizing over $\mathrm{CCSNe}$ and reducing the data to a set of distance measurements at 25 redshifts (log-spaced in the range $0.01<z<0.7$ ) and (2) using those distances, redshifts, uncertainties, and covariances to infer cosmological parameters with the cosmological Monte Carlo software (CosmoMC; Lewis \& Bridle 2002). CosmoMC allows us to easily include the latest $\mathrm{CMB}, \mathrm{BAO}$, and/or $\mathrm{H}_{0}$ priors in our cosmological constraints. This two-step procedure is similar to that of B14 (see their Appendix E).

\subsection{The Likelihood Model}

The SN likelihood model used here is discussed and tested comprehensively in J17 and is based on the BEAMS algorithm presented in Kunz et al. (2007). ${ }^{21}$ We summarize the model below.

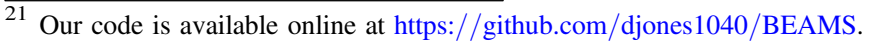


Table 4

Free Parameters in the Likelihood Model

\begin{tabular}{lccl}
\hline \hline & $\mathrm{N}_{\text {params }}$ & Prior & Comments \\
\hline$f\left(z_{b, \text { Ia }}\right)$ & 25 & $\cdots$ & $z$-dependent model of SN Ia corrected magnitudes \\
$g\left(z_{b, \mathrm{CC}}\right)$ & 5 & $2 \pm 3+\left(\mu_{\Lambda \mathrm{CDM}}\left(z_{b, \mathrm{CC}}\right)-\mathcal{M}\right)$ & $z$-dependent model of CC SN corrected magnitudes \\
$\Sigma_{\text {Ia }}$ & 1 & $0.1 \pm 0.1$ & SN Ia dispersion \\
$\Sigma_{\mathrm{CC}}\left(z_{b, c c}\right)$ & $2 \pm 2$ & CC SN dispersion \\
$\Delta_{M}$ & 5 & $0.07 \pm 0.07$ & Host mass step \\
$\alpha$ & 1 & $0.155 \pm 0.05$ & SALT2 nuisance parameter $\alpha$ \\
$\beta$ & 1 & $2.947 \pm 0.50$ & SALT2 nuisance parameter $\beta$ \\
$A$ & 1 & $1.0 \pm 0.2$ & Renormalization parameter for P(Ia) \\
$S$ & 1 & $0.0 \pm 0.2$ & Shift parameter for P(Ia) \\
\hline
\end{tabular}

Note. List of free parameters and their priors in the BEAMS likelihood model. Here $z_{b, \text { Ia }}$ denotes redshift control points for the SN Ia model, and $z_{b, \mathrm{CC}}$ denotes redshift control points for the CC SN model. The central values of the $\alpha$ and $\beta$ priors are the best-fit values using PS1 spectroscopically confirmed SNe Ia alone.

To measure distances from $\mathrm{SNe}$ Ia, we sample a posterior distribution $P(\theta \mid D)$ that is proportional to a set of priors $P(\theta)$ and the product (over $N \mathrm{SNe}$ ) of the likelihoods of the model given the data for each individual $\mathrm{SN}$. Here $D$ is the data, while $\theta$ is the set of free parameters in the model. The specific free parameters comprising $\theta$ are discussed in the paragraphs below and summarized in Table 4.

We use a three-Gaussian form of the $\mathrm{SN}$ likelihood, $\mathcal{L}$. The $\mathrm{SNe}$ Ia are represented by two Gaussians: one for SNe Ia in lowmass hosts, $\mathcal{L}_{i}^{\text {Ia }, M<10}$, and one for SNe Ia in high-mass hosts, $\mathcal{L}_{i}^{\mathrm{Ia}, M>10}$. The CC SNe are represented by the third Gaussian, $\mathcal{L}_{i}^{\mathrm{CC}}$ (alternative CC SN models are given in Section 4.5),

$$
\begin{aligned}
P(\theta \mid D) \propto & P(\theta) \times \prod_{i=1}^{N}\left(\mathcal{L}_{i}^{\mathrm{Ia}, M<10}+\mathcal{L}_{i}^{\mathrm{Ia}, M>10}+\mathcal{L}_{i}^{\mathrm{CC}}\right), \\
\mathcal{L}_{i}^{\mathrm{Ia}, M<10}= & \frac{P_{i}(M<10) P_{i}(\mathrm{Ia})}{\sqrt{2 \pi\left(\sigma_{i, \mathrm{Ia}}^{2}+\Sigma_{\mathrm{Ia}}^{2}\right)}} \\
& \times \exp \left[-\frac{\left(m_{i, \mathrm{Ia}}^{\text {corr }}+\Delta_{M}-f\left(z_{i}\right)\right)^{2}}{2\left(\sigma_{i, \mathrm{Ia}}^{2}+\Sigma_{\mathrm{Ia}}^{2}\right)}\right], \\
\mathcal{L}_{i}^{\mathrm{Ia}, M>10}= & \frac{P_{i}(M>10) P_{i}(\mathrm{Ia})}{\sqrt{2 \pi\left(\sigma_{i, \mathrm{Ia}}^{2}+\Sigma_{\mathrm{Ia}}^{2}\right)}} \\
& \times \exp \left[-\frac{\left(m_{i, \mathrm{Ia}}^{\text {corr }}-f\left(z_{i}\right)\right)^{2}}{2\left(\sigma_{i, \mathrm{Ia}}^{2}+\Sigma_{\mathrm{Ia}}^{2}\right)}\right] \\
\mathcal{L}_{i}^{\mathrm{CC}}= & \frac{P_{i}(\mathrm{CC})}{\sqrt{2 \pi\left(\sigma_{i, \mathrm{CC}}^{2}+\Sigma_{\mathrm{CC}}\left(z_{i}\right)^{2}\right)}} \\
& \times \exp \left[-\frac{\left(m_{i, \mathrm{CC}}^{\text {corr }}-g\left(z_{i}\right)\right)^{2}}{2\left(\sigma_{i, \mathrm{CC}}^{2}+\Sigma_{\mathrm{CC}}\left(z_{i}\right)^{2}\right)}\right] .
\end{aligned}
$$

Here $m_{i, \mathrm{Ia}}^{\text {corr }}$ and $m_{i, \mathrm{CC}}^{\text {corr }}$ (in the exponential terms) are shape- and color-corrected magnitudes for the $i$ th $\mathrm{SN}$ that we compute from the SALT2 parameters $m_{B}, x_{1}, c$, and $\Delta_{B}$ using the Tripp estimator. They are functions of nuisance parameters $\alpha$ and $\beta$ (Equation $\left.(1) ; m_{i, \text { Ia }}^{\text {corr }}=\mu_{i}+\mathcal{M}\right)$. Because we only wish to measure SALT2 nuisance parameters from SNe Ia, we allow separate values of $\alpha$ and $\beta$ in the Ia and CC components of the likelihood. The $m_{i, \text { Ia }}^{\text {corr }}$ values are computed using free parameters $\alpha_{\mathrm{Ia}}$ and $\beta_{\mathrm{Ia}}$. The $m_{i, \mathrm{CC}}^{\text {corr }}$ values use $\alpha_{\mathrm{CC}}$ and $\beta_{\mathrm{CC}}$, which are fixed to the values for SNe Ia given by B14 (allowing these to be free parameters does not improve the cosmological results). The parameters $\sigma_{i, \mathrm{Ia}}$ and $\sigma_{i, \mathrm{CC}}$ are the uncertainties on the corrected magnitudes of the $i$ th $\mathrm{SN}$ using $\left(\alpha_{\mathrm{Ia}}, \beta_{\mathrm{Ia}}\right)$ or $\left(\alpha_{\mathrm{CC}}\right.$, $\left.\beta_{\mathrm{CC}}\right)$, respectively.

The mass step, $\Delta_{M}$, is a free parameter that adjusts the $m_{i, \mathrm{Ia}}^{\text {corr }}$ of SNe Ia in low-mass hosts to match those in high-mass hosts. In the $\mathcal{L}_{i}^{\mathrm{Ia}, M<10}$ and $\mathcal{L}_{i}^{\mathrm{Ia}, M>10}$ terms in Equation (3), $P_{i}(M>10)$ and $P_{i}(M<10)=1-P_{i}(M>10)$ are the probabilities from host masses and host mass measurement uncertainties that a given $\mathrm{SN}$ has a host galaxy with mass $>10$ or $<10$ dex, respectively. We treat the uncertainties as Gaussian, an approximation that predominantly affects only the minority $(\sim 25 \%)$ of $\mathrm{SNe}$ that have host masses within $1 \sigma$ of $\log \left(M_{*} / M_{\odot}\right)=10$. In previous cosmological analyses (e.g., B14), the uncertainties on $\log \left(M_{*} / M_{\odot}\right)$ were neglected.

If the $\mathrm{SN}$ host galaxy has been misidentified, this could contribute to the systematic uncertainties on cosmological parameters. But for the photometrically classified sample, misidentified host galaxies would have incorrect redshifts and are therefore treated as part of the contaminating distribution $\left(\mathcal{L}_{i}^{\mathrm{CC}}\right)$. They then contribute to the "contamination" systematic, as discussed in J17. For spectroscopically classified $\mathrm{SNe}$ without host galaxy redshifts, we expect only $\sim 2 \mathrm{SNe}$ Ia to have misidentified host galaxies (based on the $1.2 \% \pm 0.5 \%$ fraction of mismatched host galaxies computed in J17).

The variable of interest for cosmological parameter estimation is $f\left(z_{i}\right)$. It is the continuous, $z$-dependent model for the SN Ia corrected magnitudes - the mean of the SN Ia Gaussian - and is allowed to vary across the redshift range of the survey $(0.01<z<0.7)$. We evaluate the model at any $z$ across this redshift range by choosing a fixed set of 25 log-spaced redshift "control points" $\left(z_{b} ; \Delta \log _{10}(z)=0.077\right)$ at which the corrected SN Ia magnitudes $f\left(z_{b}\right)=\mu\left(z_{b}\right)+\mathcal{M}$ are free parameters. For any redshift $z_{i}$, we interpolate between the redshift control points below $\left(z_{b}\right)$ and above $\left(z_{b+1}\right)$,

$$
\begin{aligned}
\mu\left(z_{i}\right) & =(1-\xi) \mu_{b}+\xi \mu_{b+1} \\
\xi & =\log \left(z_{i} / z_{b}\right) / \log \left(z_{b+1} / z_{b}\right),
\end{aligned}
$$

where $\mu_{b}$ is the distance modulus at redshift $z_{b}$. Interpolating with a simple linear model instead of $\Lambda$ CDM produces differences of $<1 \mathrm{mmag}$ at all redshifts. The SN Ia dispersion 
$\Sigma_{\text {Ia }}$ plays the same role as the intrinsic dispersion and is kept fixed at all redshifts.

The $z$-dependent mean and standard deviation of the CC SN Gaussian model $\left(g\left(z_{i}\right)\right.$ and $\left.\Sigma_{\mathrm{CC}}\left(z_{i}\right)\right)$ are interpolated between five log-spaced redshift control points. Unlike SNe Ia, the dispersion of the heterogeneous CC SN population changes with redshift due to strong detection biases at high $z$.

Each Gaussian is multiplied by the prior probability $\left(\mathrm{P}_{\mathrm{i}}(\mathrm{Ia})\right.$ and $\left.\mathrm{P}_{\mathrm{i}}(\mathrm{CC})=1-\mathrm{P}_{\mathrm{i}}(\mathrm{Ia})\right)$ that a given $\mathrm{SN}$ is or is not of Type Ia. We use the PSNID classifier to estimate these probabilities. Alternative classification methods are included as part of our systematic error budget (Section 4.5).

For $\mathrm{SNe}$ with photometric classifications, our method allows the type priors to be shifted and renormalized to account for incorrect classifications (see J17). For spectroscopically classified $\mathrm{SNe} I a$, we set the prior probabilities, $\mathrm{P}_{\mathrm{i}}(\mathrm{Ia})$, equal to 1 and do not allow them to be adjusted. We include broad Gaussian priors (Table 4) on all free parameters with the exception of $f\left(z_{b}\right)$, the SN Ia corrected magnitudes. We apply no priors (i.e., flat priors) to $f\left(z_{b}\right)$ to avoid any possibility of cosmological bias.

We estimate the free parameters by sampling the log of the posterior with a Markov chain Monte Carlo (MCMC) algorithm. As in J17, we use the Parallel-Tempered Ensemble Sampler from emcee as our MCMC method (ForemanMackey et al. 2013).

\subsection{Constraining Cosmological Parameters}

From the methods presented above, we infer the corrected magnitudes of SNe Ia at 25 redshift control points, $f\left(z_{b}\right)$, using the baseline SN light-curve parameters, bias corrections, and J17 methodology. We also measure the set of $f\left(z_{b}\right)$ for each systematic uncertainty (Section 4). From these values, a systematic error covariance matrix $C_{\text {sys }}$ is created (Conley et al. 2011; Scolnic et al. 2014):

$$
C_{\mathrm{sys}}^{j k}=\sum_{n=1}^{N} \frac{\partial f\left(z_{j}\right)}{\partial S_{n}} \frac{\partial f\left(z_{k}\right)}{\partial S_{n}} \sigma\left(S_{n}^{2}\right) .
$$

The sum is over all $N$ systematics, and $\frac{\partial f\left(z_{j}\right)}{\partial S_{n}}$ is the change in corrected magnitude after applying a single systematic $S_{n}$ to the individual light curves. Here $\sigma\left(S_{n}\right)$ is the size of each systematic uncertainty. The systematic covariance matrix is then combined with the statistical covariance matrix:

$$
C_{\mathrm{tot}}=D_{\text {stat }}+C_{\text {sys }}
$$

Note that the statistics-only covariance matrix, $D_{\text {stat }}$, includes both diagonal and off-diagonal components because the magnitudes $f\left(z_{b}\right)$ are anticorrelated with the neighboring magnitudes $f\left(z_{b+1}\right)$ and $f\left(z_{b-1}\right)$ :

$$
D_{\text {stat }}^{i j}=\sum_{k}^{N_{\mathrm{MCMC}}} \frac{\left(f_{k}\left(z_{b, i}\right)-\overline{f\left(z_{b, i}\right)}\right)\left(f_{k}\left(z_{b, j}\right)-\overline{f\left(z_{b, j}\right)}\right)}{N_{\mathrm{MCMC}}} .
$$

Here $N_{\mathrm{MCMC}}$ is the length of the MCMC chain that samples free parameters $f\left(z_{b}\right), f_{k}\left(z_{b, i}\right)$ is the value of $f$ at the $i$ th control point from the $k$ th MCMC sample, and $\overline{f\left(z_{b, i}\right)}$ is the mean of $f$ at the $i$ th control point from the full MCMC chain. Figure 6 shows the reduced correlation matrices from statistical uncertainties alone (left) and statistical and systematic uncertainties combined (right). The statistics-only correlation matrix shows significant anticorrelations between neighboring control points, while the systematic uncertainties add largerscale correlations between the control points (see Figure 7).

We then use the CosmoMC software (Lewis \& Bridle 2002) to measure cosmological parameters by minimizing the following $\chi^{2}$ :

$$
\begin{aligned}
\chi^{2}= & \left(\mu^{\prime}\left(z_{b}\right)-\mu_{\Lambda \mathrm{CDM}}\left(z_{b} ; \Omega_{M}, w, \ldots\right)\right)^{\dagger} C_{\mathrm{tot}}^{-1} \\
& \times\left(\mu^{\prime}\left(z_{b}\right)-\mu_{\Lambda \mathrm{CDM}}\left(z_{b} ; \Omega_{M}, w, \ldots\right)\right),
\end{aligned}
$$

where $\mu^{\prime}\left(z_{b}\right)=f\left(z_{b}\right)-\mathcal{M}$ (we marginalize over $\mathcal{M}$ using CosmoMC). The vector of model distances, $\mu_{\Lambda \mathrm{CDM}}=$ $5 \log \left(d_{L}\right)-5$, is a function of the cosmology:

$$
\begin{aligned}
& d_{L}\left(z, w, \Omega_{M}, \Omega_{\Lambda}, \Omega_{K}\right)=(1+z) \frac{c}{H_{0}} \int_{0}^{z} \frac{d z}{E(z)}, \\
& E(z)=\left[\Omega_{M}(1+z)^{3}+\Omega_{k}(1+z)^{2}+\Omega_{\Lambda}(1+z)^{3(1+w)}\right]^{1 / 2} .
\end{aligned}
$$

The parameter $\Omega_{M}$ is the cosmic matter density, $\Omega_{\Lambda}$ is the dark energy density, $\Omega_{k}$ is the curvature of space, and $w$ is the redshift-independent dark energy equation-of-state parameter ( $z$ dependence will be added in Section 6).

\section{Systematic Uncertainties}

The SNe in this sample are affected by systematic uncertainties that can broadly be attributed to eight sources of error: Milky Way extinction, distance bias correction, photometric calibration, SALT2 model calibration, sample contamination (primarily by $\mathrm{CC} \mathrm{SNe),} \mathrm{low-z} \mathrm{peculiar-velocity} \mathrm{corrections,} \mathrm{the} \mathrm{redshift} \mathrm{depend-}$ ence of SN nuisance parameters, and the dependence of SN Ia luminosities on their host galaxies. Figure 7 illustrates the redshift dependence of each type of systematic uncertainty. We discuss each of these uncertainties in detail below.

\subsection{Milky Way Extinction}

Milky Way extinctions for each SN are given by Schlafly \& Finkbeiner (2011), who used the colors of stars with spectra in SDSS to derive a $14 \%$ correction to the reddening maps of Schlegel et al. (1998). We assume a conservative, fully correlated $5 \%$ uncertainty on the $E(B-V)$ measurements of Schlafly \& Finkbeiner (2011), which could be caused by selection biases in the SDSS stars chosen for spectroscopic follow-up or the use of stars that lie in front of some fraction of the Galactic dust (Schlafly \& Finkbeiner 2011).

\subsection{Distance Bias Correction}

Two effects lead to systematic uncertainties in distance bias corrections. The dominant effect is the difference between the G10 and C11 distance bias predictions. As discussed in Section 2.3.2, the difference between the $\mathrm{G} 10$ and $\mathrm{C} 11$ dispersion models is up to $\Delta \mu(z) \sim 0.03 \mathrm{mag}$. As there is no a priori reason to choose one dispersion model over the other, 
corr. coeff.
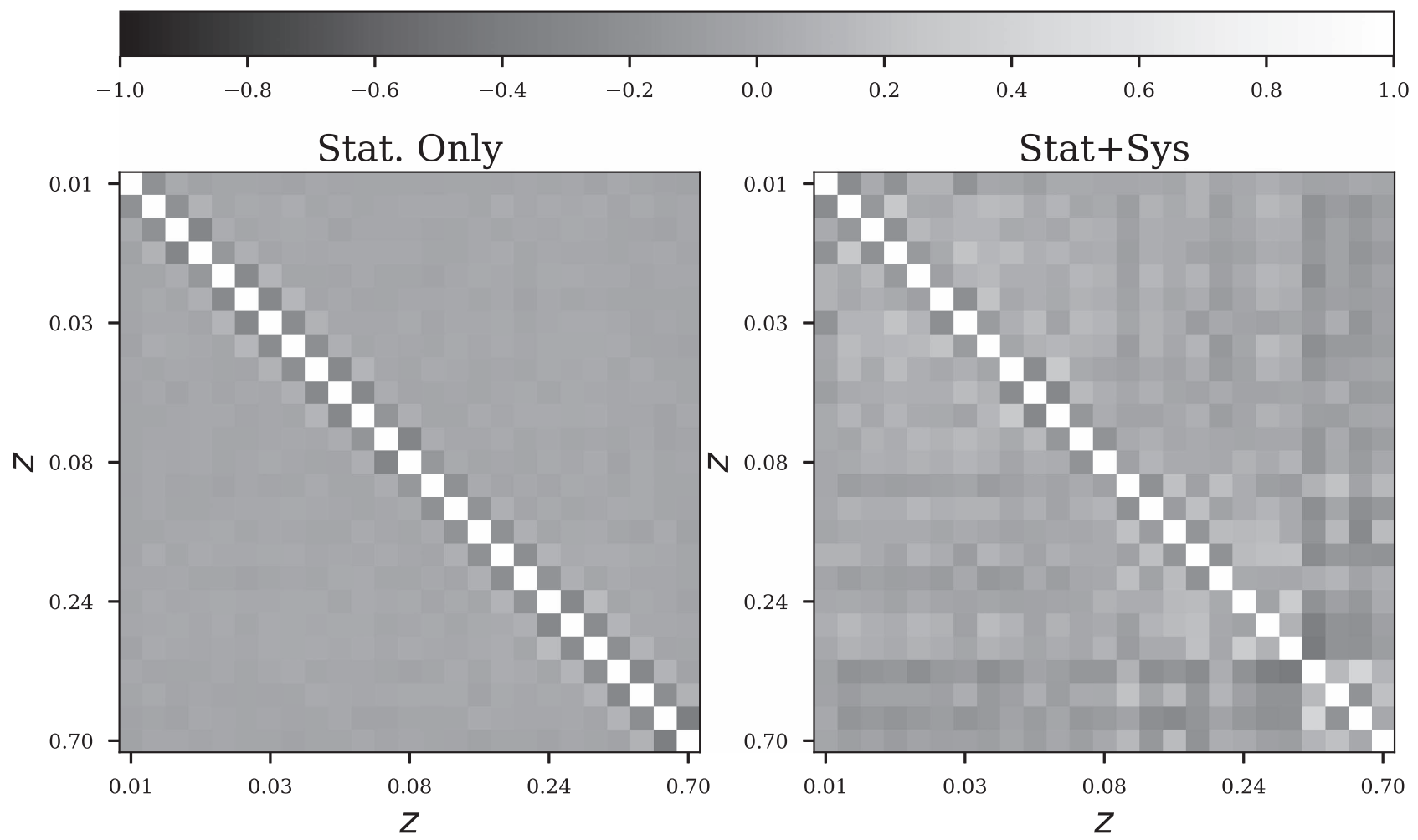

Figure 6. Statistics-only and stat + sys correlation matrices from the PS1+low- $z$ SN sample. The statistics-only correlation matrix shows the strong anticorrelation between neighboring bins. The stat+sys correlation matrix shows larger-scale correlations due to systematic uncertainties and large uncertainties in the bins with minimal data $(z \sim 0.1-0.2)$. The correlation matrix is equal to $C_{i j} / \sqrt{C_{i i} C_{j j}}$ for covariance matrix $C$.

we choose to adopt the average of the two bias predictions for our baseline distance bias correction. The systematic error then becomes half the difference between the G10/C11 bias.

A secondary effect is that uncertainty in the survey detection limit or spectroscopic follow-up selection function can cause the simulated distance bias to be inaccurate. We adjust the detection efficiency (for the PS1 host- $z$ sample) and the spectroscopic selection efficiency (for the PS1 SN-z sample) such that the $\mathrm{S} / \mathrm{N}$ at maximum light for simulated $\mathrm{SNe}$ matches the data with an $\sim 20 \%$ higher reduced $\chi^{2}$ (a $1 \sigma$ difference). These efficiencies are well-constrained by the data; the detection efficiency adjustment for the host $z$ sample, for example, corresponds to lowering the magnitude limit of the survey by $\sim 4$ mmag.

The low- $z$ distance bias is measured from low $z$ s simulations that lack reliable detection and spectroscopic selection efficiencies. For these simulations, we use the "volume-limited" simulations discussed in Section 2.3.1 as the selection bias systematic. The volume-limited variant has $<0.01 \mathrm{mag}$ distance bias using the G10 scatter model (small biases due to the correlation of Hubble residuals with $x_{1}$ and $c$ still arise; Scolnic $\&$ Kessler 2016) and a bias of $\sim 0.02$ mag using the C11 model because $\beta_{\text {fit }}-\beta_{\text {sim }}=0.7$. The systematic uncertainty due to the detection limit and spectroscopic follow-up selection function is subdominant to the G10/C11 systematic uncertainty.

\subsection{Photometric Calibration Uncertainties}

In this work, the systematic uncertainties in the photometric calibration are the same as those in the S17 analysis. They are due to uncertainties in the survey filter functions, calibration of HST CALSPEC standard stars, and calibration of the PS1/low$z$ photometric systems relative to $H S T$.

Uncertainties in the survey filter functions are modeled as uncertainties in the zero points and effective wavelengths of each filter. PS1 has a effective central wavelength uncertainty of $7 \AA$ per filter (Scolnic et al. 2015). The low- $z$ filter uncertainties are typically $\sim 6-7 \AA$ but are survey- and filterdependent. They can be as high as $25-37 \AA$ (exact values are given in Scolnic et al. 2015; see their Table 1).

The relative calibration uncertainties are given by the Supercal method. Supercal uses the excellent (sub-1\%) relative calibration of PS1 across $3 \pi$ sr to compare the photometry of tertiary standard stars in previous SN surveys to the photometry of these same stars on the PS1 system. Typical corrections are on the order of $1 \%$ but can be up to $2.5 \%$ for $B$-band low- $z$ data. Uncertainties in the Supercal procedure are typically 3-4 mmag per filter but can be up to 10 mmag for low-z surveys such as CfA1.

Finally, there is uncertainty in the $\mathrm{AB}$ magnitude system itself, as measured using HST CALSPEC standard stars. We follow B14 by assuming a global $0.5 \%$ slope uncertainty for the flux as a function of wavelength, which was determined by comparing white dwarf models to the HST data (Betoule et al. 2013; Bohlin 2014). In total, we include 62 individual systematic uncertainties to describe the uncertainty in the photometric calibration. Most are due to the relative calibration: there is one systematic for the filter zero point and the filter $\lambda_{\text {eff }} \times$ number of surveys $\times$ number of filters per survey. 


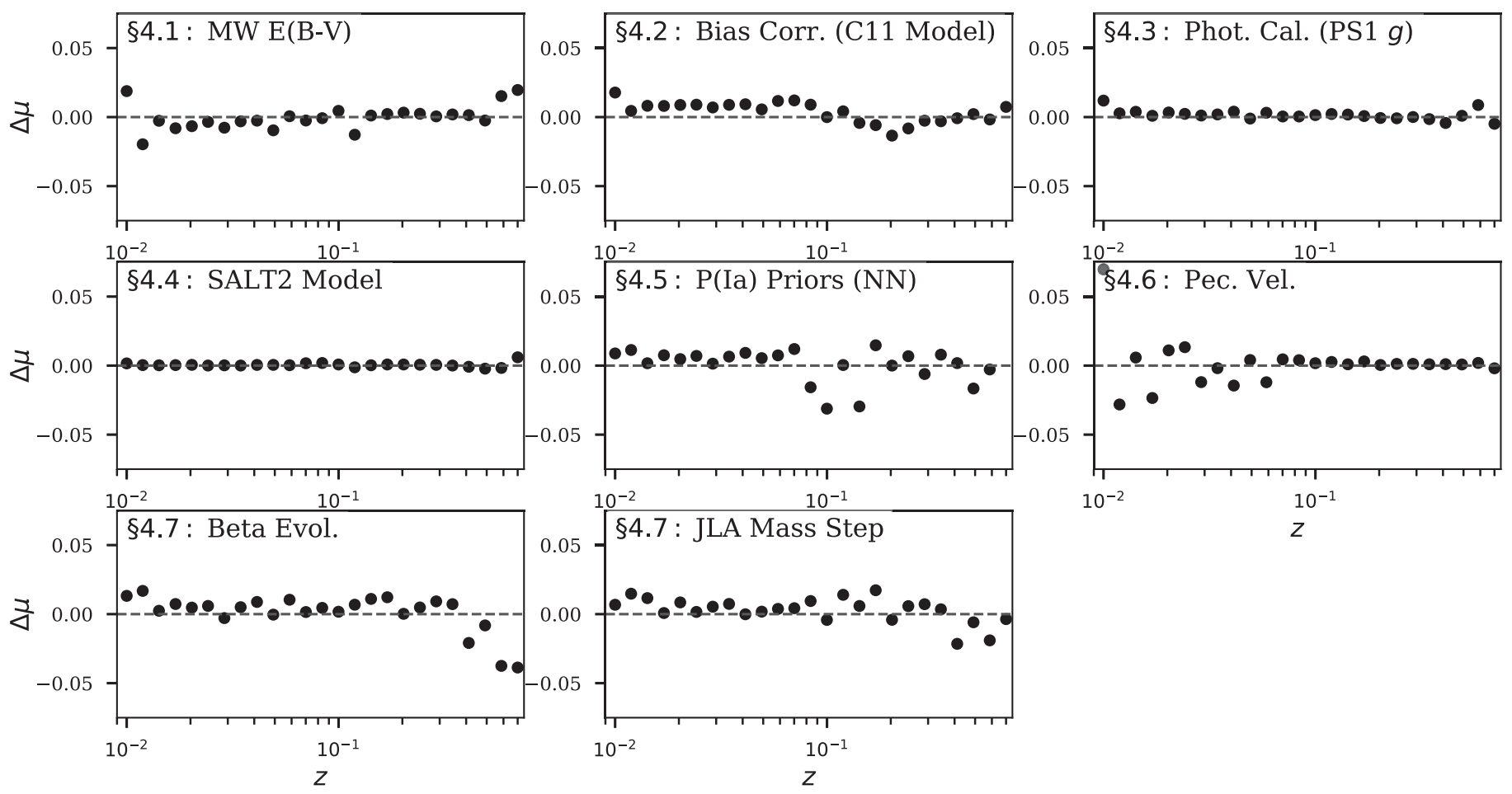

Figure 7. Average change in distance modulus $\Delta \mu$ from an example of each type of systematic uncertainty in this analysis. Deviations at $z \simeq 0.01$ and $\simeq 0.1$ are primarily due to low SN statistics in these bins and have little effect on the cosmological constraints.

\subsection{SALT2 Model Calibration Uncertainties}

The training of the SALT2 model is subject to the same sources of photometric calibration uncertainty discussed above. B14 created variants of the SALT2.4 light-curve model by applying zero-point and filter function shifts to the training data and subsequently retraining SALT2. These account for 10 individual systematics, which are averaged to give the SALT2 model systematic error. These uncertainties are discussed in Section 5.4 of B14.

Retraining SALT2 using the improved calibration from Supercal will lower the SALT2 systematic uncertainty in future analyses. However, we do not retrain the SALT2 light-curve model for this analysis, as the SALT2 training data are not public.

\subsection{SN Contamination}

Systematic error due to marginalizing over the contamination in our sample is a new source of uncertainty caused by our use of photometrically classified SNe. J17 predicted that the PS1 host- $z$ sample contains $\sim 9 \%$ CC SNe. Our method of measuring distances from SNe Ia while marginalizing over CC SNe is subject to biases in two areas: inaccurate prior probabilities that a given SN is of Type Ia and differences between the CC SN model and the true distribution of CC SNe. The systematic error estimation from CC SN contamination was presented in detail in $\mathrm{J} 17$ and relies on varying these components.

We use the four methods of estimating prior probability discussed previously (Section 2.4) and three parametric models for the CC SN distribution. The baseline likelihood model for $\mathrm{CC} \mathrm{SNe}, \mathcal{L}_{i}^{\mathrm{CC}}$ (Equation (3)), is a Gaussian with a mean and standard deviation $-g\left(z_{i}\right)$ and $\Sigma_{\mathrm{CC}}\left(z_{i}\right)$ for the $i$ th $\mathrm{SN}$-that are both functions of redshift. The two alternate CC SN parametric models are a two-Gaussian model and a skewed Gaussian model. We demonstrated in J17 that these models typically agree well with single-Gaussian results; all three CC SN distributions tend to be much broader than the SN Ia distribution, therefore encompassing most outliers regardless of whether the functional form is an exact representation of the CC SN data.

Because several of these variants are highly covariant with one another, we group the different contamination variants into two systematics: one using the results from SN classifiers trained on simulated CCSN data and a second using "untrained" classifiers. The trained classifiers include NN and PSNID. The trained systematic is the average change in SN distances when either the NN or PSNID classifier is used with alternate CC SN models. Fitprob and GalSNID are not trained on simulations, and so we include the average of the Fitprob and GalSNID distances as a second systematic. The untrained classifiers are not optimal methods but are included here as an alternative to classifiers that depend on simulations with limited CC SN templates and known biases. If each variant were instead treated as an individual systematic, our final uncertainty would only increase by $2 \%$, and the final value of $w$ would be higher by just 0.003 .

Finally, we found in J17 that BEAMS can yield results with less bias if $\alpha$ and $\beta$ are fixed to their known values from spectroscopically classified samples. For a single-Gaussian CC SN model with PSNID, we include this variant in our systematic uncertainty budget by forcing $\alpha$ and $\beta$ to be equal to the values measured from spectroscopically confirmed PS1 + low- $z$ SNe. The shape and color distributions in the full PS1 sample are different than those in the PS1 spectroscopically classified sample, which could mean that $\alpha$ and $\beta$ are in fact not the same in the full sample as in the spectroscopically classified sample (Scolnic \& Kessler 2016). However, because it is not possible to distinguish between true differences in $\alpha / \beta$ and differences caused by the known $\alpha / \beta$ biases when 
marginalizing over $\mathrm{CCSNe}(\mathrm{J} 17)$, this variant is a necessary addition to the error budget.

\subsection{Peculiar-velocity Correction}

The magnitude of SN peculiar velocities, due to bulk flows and nearby superclusters, becomes $\gtrsim 5 \%$ of the Hubble flow at $z \lesssim 0.03$. We correct for peculiar velocities using the nearby galaxy density field measured by the $2 \mathrm{M}++$ catalog from 2MASS (Lavaux \& Hudson 2011). The uncorrelated uncertainty associated with each correction is $\pm 250 \mathrm{~km} \mathrm{~s}^{-1}$ (S17). The peculiar-velocity model is parameterized by the equation $\beta_{I}=\Omega_{M}^{0.55} / b_{I}$, where $b_{I}$ describes the light-to-matter bias. (The parameter $\beta_{I}$ is unrelated to the SALT2 nuisance parameter.) Carrick et al. (2015) measured $\beta_{I}=0.43 \pm 0.021$. We adopt a conservative $5 \sigma( \pm 0.1)$ systematic on $\beta_{I}$ for our peculiarvelocity systematic uncertainty.

\subsection{SN Ia Demographic Shifts}

Though SNe Ia have been shown to be excellent standardizable candles at low- $z$, it has been suggested that the relationship between their luminosities, colors, and host galaxy properties may change with redshift. We address these possibilities by adding three systematic tests. For these tests, we add additional parameters to our model for estimating cosmological parameters (Section 3). The first is to allow a linear evolution of the mass step as a function of redshift. Mass step evolution was proposed by Childress et al. (2014) and could be observed if the mass step is caused by physical differences in SNe Ia with different progenitor ages. The second is to allow a linear evolution in the SALT2 color-standardization parameter, $\beta$, as a function of redshift. This was suggested as a possible concern by Conley et al. (2011). The third is evolution in SALT2 $\alpha$. Then, $\Delta_{M}, \alpha$, and $\beta$ in Equations (1) and (3) become

$$
\begin{aligned}
\Delta_{M} & =\Delta_{M, 0}+\Delta_{M, 1} \times z, \\
\alpha & =\alpha_{0}+\alpha_{1} \times z, \\
\beta & =\beta_{0}+\beta_{1} \times z .
\end{aligned}
$$

Here $\Delta_{M, 0}, \Delta_{M, 1}, \beta_{0}, \beta_{1}, \alpha_{0}$, and $\alpha_{1}$ are free parameters. They are measured simultaneously with SN Ia distances in Section 5. Because we find no hint of Mass step evolution or $\alpha$ evolution, we include only $\beta$ evolution as a systematic uncertainty in our final measurement (see Section 5).

We also include a $\Delta_{M}$ variant that shifts the divide between "low-mass" and "high-mass" hosts by 0.15 dex relative to the standard divide at $\log \left(M_{*} / M_{\odot}\right)=10$, following the uncertainty on the location of the step measured by S17. Finally, because possible bias in $\Delta_{M}$ due to marginalizing over CC SN contamination was not estimated in $\mathrm{J} 17$, we add one variant where $\Delta_{M}$ is fixed to the value measured by B14 $(0.07 \pm 0.023 \mathrm{mag})$.

We note that because our sample preferentially contains bright host galaxies, our results are sensitive to uncertainty in the relation between host galaxy properties and SN luminosity. However, because most low- $z$ SNe originate from SN searches that specifically targeted bright galaxies, the PS1 photometric data are in some ways more similar to the existing low- $z$ data than to previous high- $z$ data sets. In this way, our results might be less biased by the uncertainty in the relationships between $\mathrm{SNe}$ and their host galaxies than those of previous analyses.

An additional potential systematic is the relation between SN Ia corrected magnitudes and their local host galaxy environments. Several papers have recently asserted that SN Ia corrected magnitudes are correlated with their local star formation environments on a scale of $\sim 1-3 \mathrm{kpc}$ (the LSF step; Rigault et al. 2013, 2015). Due to the $\sim 1^{\prime \prime}$ PSF of PS1 and the lack of ultraviolet or $u$-band observations for much of our sample, it is impossible to measure robust local star formation rates over the PS1 redshift range. However, Jones et al. (2015) reexamined the evidence for the LSF step, finding that the retraining of SALT2 in B14/G10 reduced or eliminated many of the biases in the SALT2 model. Jones et al. (2015) found no evidence for an LSF step in the B14 low-z sample. Roman et al. (2017) also recently measured a strong dependence of SN Ia luminosities on local $U-V$ color but found that this effect is expected to change $w$ by just 0.006 relative to the standard $\Delta_{M}$ correction. Though our data are not optimal for investigating local properties, we plan to use PS1 data to more robustly determine the relationship between $\mathrm{SNe}$ Ia and their global or semi-local host galaxy properties in future work.

\section{First Results and Consistency Checks}

The PS1+low- $z$ Hubble diagram is shown in Figure 8, and the light-curve parameters for our full sample are given in Table 2 . There are $\sim 3.5$ times as many photometrically classified $\mathrm{SNe}$ as there are spectroscopically classified SNe. The binned SN Ia distance uncertainties from the full sample are an average of $40 \%$ lower than those from spectroscopically classified SNe Ia alone (statistical uncertainties only). At $0.2<z<0.5$, where $\sim 75 \%$ of the PS1 data lie, uncertainties are $\sim 45 \%$ lower. This is in spite of the fact that the photometrically classified SNe have a lower average $\mathrm{S} / \mathrm{N}$; the median $\mathrm{S} / \mathrm{N}$ at peak is 22 for all PS1 SNe, compared to 38 for spectroscopically classified $\mathrm{SNe}$. We also do not expect that marginalizing over CC SNe has inflated the binned distance uncertainties. In $\mathrm{J} 17$, we used simulated data to find that our method of marginalizing over CC SNe increases the statistical uncertainty on binned SN Ia distances by just 3\%.

Our likelihood model (Equation (3)) is simultaneously used to measure $\alpha, \beta$, and the dispersion $\Sigma_{\mathrm{Ia}}$, which are given in Table 5. These measurements use the baseline classification method, PSNID, and the one-Gaussian CC SN model, while the alternate methods contribute to the systematic errors in the middle column. We measure $\alpha=0.165 \pm 0.019$ (stat + sys), which is consistent with the value measured by $\mathrm{S} 17$ from low- $z$, PS1, SDSS, and SNLS spectroscopically confirmed SNe $(\alpha=0.156 \pm 0.006)$. Zhang et al. (2017) also found $\alpha=0.165 \pm 0.010$ for low- $z$ SNe. However, we note that this value is higher than that measured by B14 by $\sim 1 \sigma(\sim 2.5 \sigma$ from statistical uncertainties alone), and the reason for this difference is unclear.

The uncertainty on the SN Ia dispersion, $\Sigma_{\text {Ia }}=0.082 \pm$ 0.067 , is extremely high. This is a consequence of removing $\mathrm{P}(\mathrm{Ia})<0.5 \mathrm{SNe}$ from the sample before cosmological parameter estimation and allowing $\mathrm{SN}$ type probabilities to be shifted and renormalized by the likelihood model. If $\mathrm{P}(\mathrm{Ia})<0.5 \mathrm{SNe}$ are included, we find that $\Sigma_{\text {Ia }}$ is better constrained, with a value of $0.106 \pm 0.032$ (stat. errors only), consistent with $\Sigma_{\mathrm{Ia}}=0.118$ from spectroscopically classified $\mathrm{SNe}$ Ia alone. We note that in spite of the large uncertainty on $\Sigma_{\text {Ia }}$, the distance uncertainties are slightly smaller when $\mathrm{P}(\mathrm{Ia})<0.5 \mathrm{SNe}$ are removed. Removing $\mathrm{P}(\mathrm{Ia})<0.5 \mathrm{SNe}$ changes the statistics-only measurement of $w$ by just $0.3 \%$. 


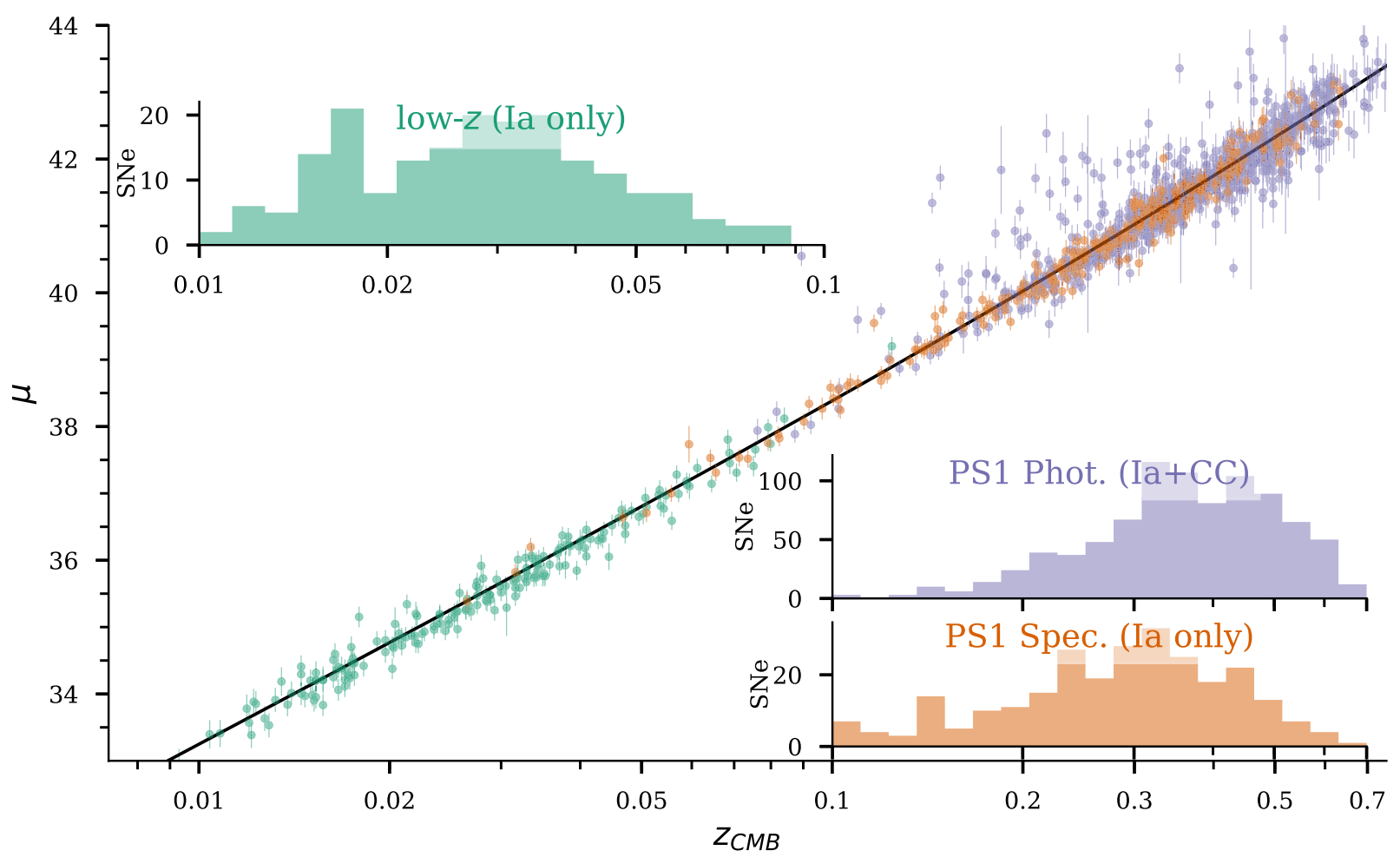

Figure 8. The PS1+low- $z$ Hubble diagram with low- $z$ SNe Ia, spectroscopically classified SNe Ia, and photometrically classified SNe. The data that appear much fainter than $\Lambda$ CDM (black line) are likely CC SN contaminants. We use 1364 SNe to measure cosmological parameters.

Table 5

Nuisance Parameters

\begin{tabular}{lcccccc}
\hline \hline & \multicolumn{3}{c}{ All SNe } & & \multicolumn{2}{c}{ Spec. Class. SNe } \\
\cline { 2 - 4 } \cline { 7 - 7 } & & $\sigma_{\text {stat }}$ & $\sigma_{\text {stat }+ \text { sys }}$ & & $\sigma_{\text {stat }}$ \\
\hline$\alpha$ & 0.165 & 0.006 & 0.019 & & 0.155 & 0.009 \\
$\beta$ & 3.028 & 0.067 & 0.152 & & 2.944 & 0.092 \\
$\Sigma_{\text {Ia }}$ & 0.082 & 0.067 & 0.101 & & 0.118 & 0.008 \\
$\Delta_{M}$ & 0.102 & 0.013 & $0.017^{\mathrm{a}}$ & & 0.064 & 0.020 \\
\hline
\end{tabular}

Note. Nuisance parameters from PS1+low- $z$ SNe. The systematic uncertainty on $\beta$ is likely overestimated due to the biases from the GalSNID and Fitprob classification methods discussed in J17. The exceptionally large uncertainty on $\Sigma_{\text {Ia }}$ is due to our decision to exclude $\mathrm{SNe}$ with $\mathrm{P}(\mathrm{Ia})<0.5$ and allow $\mathrm{P}(\mathrm{Ia})$ to be shifted and renormalized, but we have verified that this choice has a minimal effect on the final cosmological parameters.

${ }^{a}$ The systematic uncertainty excludes the analysis variants that change the location, size, and $z$ dependence of $\Delta_{M}$.

As a test, if the sample is analyzed without BEAMS, i.e., treating all $\mathrm{SNe}$ as $\mathrm{SNe}$ Ia, $\Sigma_{\mathrm{Ia}}$ increases by $71 \%$ to 0.187 . In general, the systematic error on all nuisance parameters is higher than it would be in an analysis of spectroscopically classified $\mathrm{SNe} \mathrm{Ia}$, due to the predicted biases on those parameters when marginalizing over CC SN contamination (J17). Fortunately, J17 found that biases of 3\%-6\% on nuisance parameters do not give similar fractional biases on binned distances or $w(0.5 \% \pm 0.4 \%$ bias on $w$ for the baseline method).

In J17, we predicted that our method of marginalizing over CC SNe would bias $\alpha$ and $\beta$ by $+3 \%$. This gives a prediction that the $\alpha$ and $\beta$ measured here will be higher than the $\alpha$ and $\beta$ measured from spectroscopically confirmed SNe Ia alone. Table 5 shows that this may indeed be the case; $\alpha$ is $6 \%$ higher and $\beta$ is $3 \%$ higher than the values from spectroscopically confirmed PS1+low- $z$ SNe Ia (though at $<1 \sigma$ significance if we neglect the partial correlations between these two samples). However, we also expect higher measured values of $\beta$ due to the redder colors of the full PS1 sample (Scolnic \& Kessler 2016).

We also measure the mass step at $6 \sigma$ significance and nearly $8 \sigma$ from statistical errors alone (we report systematic uncertainties that neglect the host mass variants). Our measurement of $\Delta_{M}=0.102 \pm 0.017$ is consistent with the B14 measurement of $0.07 \pm 0.023$. It is also consistent with the $\Delta_{M}$ that we measure from the low- $z$ sample alone, $\Delta_{M}=0.110 \pm 0.038$. Interestingly, the host mass step $\Delta_{M}$ is higher in the full PS1+low- $z$ sample than in the sample of spectroscopically classified SNe Ia alone $(1.1 \sigma$ significance from statistical uncertainties alone, though these measurements are not independent). It is unclear if this difference could be due to statistical fluctuation, a bias from the method, or the presence of broader light-curve shapes and redder colors in the full sample. Because $x_{1}$ and $c$ correlate with both the host mass and Hubble residual (Scolnic \& Kessler 2016), different $x_{1}$ and $c$ distributions could increase the size of the step (S17). We will use simulations to investigate whether our method of marginalizing over CC SN contamination could bias determinations of the host mass step in future work.

\subsection{Impact of Different Classification Methods}

Regardless of which classifier is used, uncertainties on binned distances from the full PS1 sample are much smaller than the uncertainties on binned distances from spectroscopically classified SNe Ia alone (by $\gtrsim 40 \%$ ). The binned SN Ia distance measurements from each classifier are also remarkably consistent (Figure 9). Nearly all distances are 
within $1 \sigma$ of distances derived from the PS1 spectroscopically classified SN Ia sample. Additionally, binned distances from $0.2 \lesssim z \lesssim 0.5$, where $75 \%$ of our data lie, show few discrepancies between the different methods. Even the test case of using an uninformative prior of $\mathrm{P}(\mathrm{Ia})=1 / 2$ for all photometrically classified $\mathrm{SNe}$ (bottom panel) yields distances within $1 \sigma$ of the spectroscopic sample in all bins but one. We note that close agreement is predicted by $\mathrm{J} 17$; even in a sample without spectroscopically confirmed SNe Ia, J17 predicted biases of $<10$ mmag due to the method. We will revisit this prediction in Section 8 to test whether our methodology remains robust and consistent in the case of an "ideal" photometrically classified SN sample, i.e., a sample without spectroscopic classifications.

The nuisance parameters $\alpha$ and $\beta$, as measured using different classification priors, are more consistent than expected from J17. When using different classification priors, $\alpha$ and $\beta$ vary by $30 \%-50 \%$ less than the simulation-based predictions in J17 (in this work, we observe differences of $\Delta \beta \sim 0.07$ and $\Delta \alpha \sim 0.004$ between the four different classification methods). In Figure 10, we provide a possible explanation for why our results are more consistent than expected. J17 simulations included no subset of spectroscopically classified PS1 SNe, while our data consist of $\sim 24 \%$ spectroscopically classified PS1 SNe. Because of this, we used simulations of the PS1 host$z$ and $\mathrm{SN}-z$ samples (Section 2.3.1) to predict the effect of adding spectroscopically classified subsets of $\mathrm{SNe}$ to the data. We find that the predicted biases on $\alpha$ and $\beta$ due to marginalizing over CC SNe decrease by $30 \%-40 \%$ when the PS1 data consist of $24 \%$ spectroscopically classified SNe.

Similarly, the biases on individual distance bins decrease by $\sim 30 \%-40 \%$ when $24 \%$ of PS1 $\mathrm{SNe}$ are spectroscopically classified. For PSNID priors, Figure 10 shows that the predicted (weighted) average bias in distance modulus at $z>0.1$ relative to $z<0.1$ is just 2 mmag.

\subsection{Evolution of Nuisance Parameters}

Using Equation (10) to add linear Mass step $\left(\Delta_{M}\right)$ evolution to BEAMS, we find no evolution in $\Delta_{M}$ as a function of redshift (we use the baseline classifier, PSNID). However, our uncertainties are large, $\sim 0.08 \mathrm{mag}$, due to a lack of low-mass hosts at high redshift. ${ }^{22}$ In Figure 11, we estimate the redshift dependence of the mass step with a $2.5 \sigma$ clip of Hubble residuals $(-0.45 \lesssim \mathrm{HR} \lesssim 0.45)$ to remove most CC SNe and then plot the maximum-likelihood mass step in redshift bins of 0.1 . This is an incomplete removal of CC SN contamination, but it doubles as a simple sanity check on BEAMS. We find no statistically significant evidence for Mass step evolution.

We do see $1.6 \sigma$ evidence for evolution of the $\beta$ parameter, however (Figure 12). Fortunately, this does not constitute a large contribution to our systematic error budget, as it predominantly affects the highest survey redshifts where few $\mathrm{SNe}$ are found (Figure 7). Evidence for $\beta$ evolution was seen in SNLS data (Conley et al. 2011), though its significance is attributed to selection effects in B14. S17 found just $1 \sigma$ evidence for $\beta$ evolution $(\beta=(3.139 \pm 0.099)+z \times(-0.348 \pm 0.289))$, a measurement that includes $\mathrm{SNe}$ at redshifts up to $\sim 2$ (Riess et al. 2018). Though there are not enough SNe Ia at $z>1.5$ to constrain

\footnotetext{
22 S17, however, found evidence of Mass step evolution. The discrepancy could be due to the larger SNLS redshift range and additional SNe Ia in lowmass hosts at $z>0.5$.
}

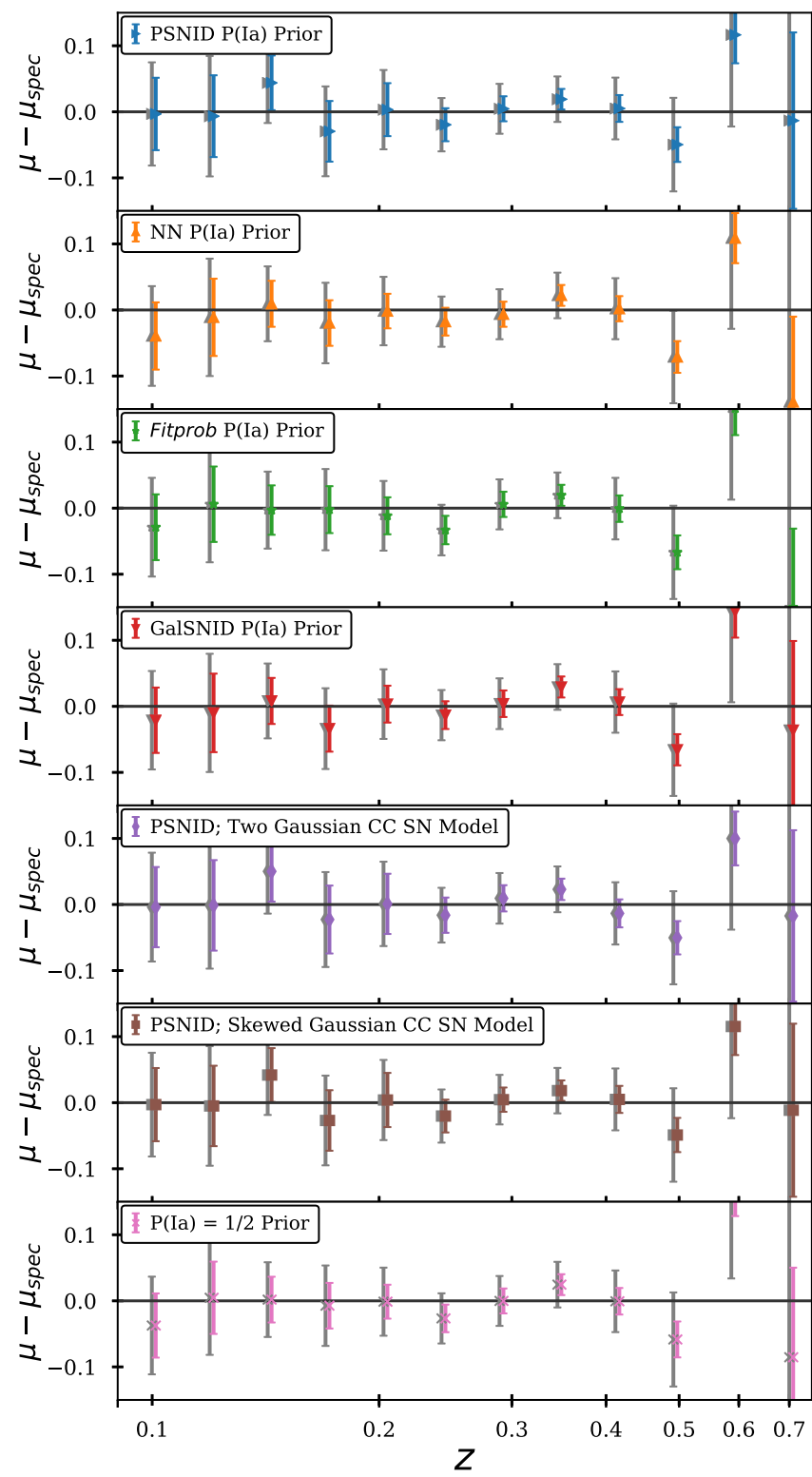

Figure 9. Difference in binned distance from the full photometric sample compared to binned distances from the subset of $\sim 280$ spectroscopically classified PS1 SNe Ia. Gray (large) error bars are the uncertainties on spectroscopic and photometric distances added in quadrature, while the smaller errors are from the photometric sample alone (small redshift offsets are added to the photometric points for visual clarity). Binned distances are consistent between methods, with a small bump at $z \sim 0.35$ that could be due to high CC SN contamination at this redshift but is also consistent with statistical fluctuation. For comparison to the predicted biases from simulations, see Figure 11 of J17.

a changing value of $\beta$, larger high- $z$ data sets may be able to confirm or discount $\beta$ evolution. We caution that blue $(c<0)$ SNe Ia have lower observed $\beta$ (SNe primarily appear blue due to noise and selection biases; Scolnic \& Kessler 2016), and our high$z$ data are dominated by blue SNe (Figure 1). However, our methodology does not recover any significant evolution of $\beta$ when tested on simulated SN samples with a constant input $\beta$. In 10 simulated SN samples, five using the G10 model and five using the $\mathrm{C} 11$ model, we found just a single sample showing $>1 \sigma$ evidence of negative $\beta$ evolution with redshift (the simulation had a $\beta$ slope with significance of $1.2 \sigma$ ). If $\beta$ does change with $z$, it could suggest an evolution in dust properties or the evolution of 

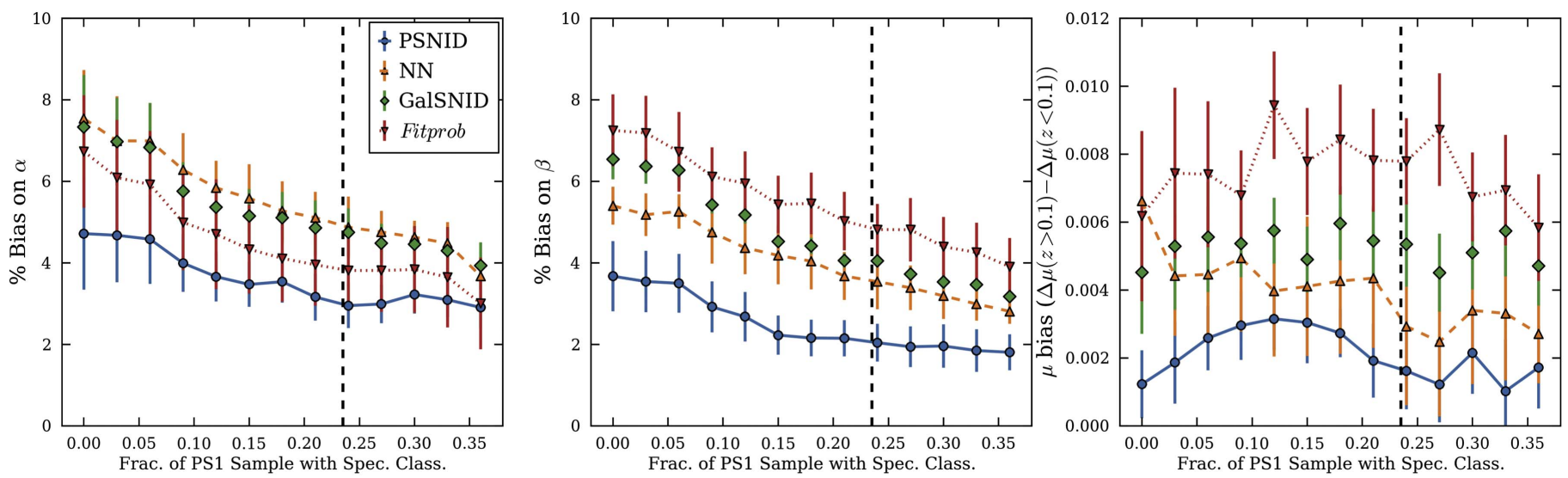

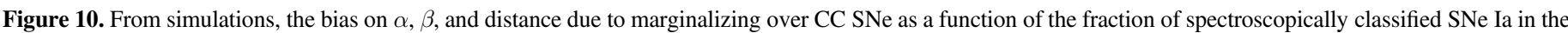

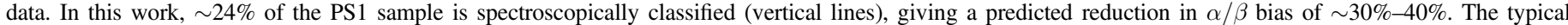

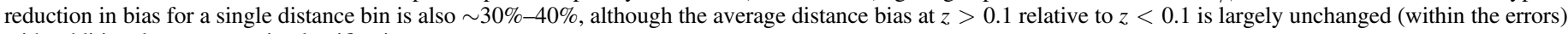
with additional spectroscopic classifications.

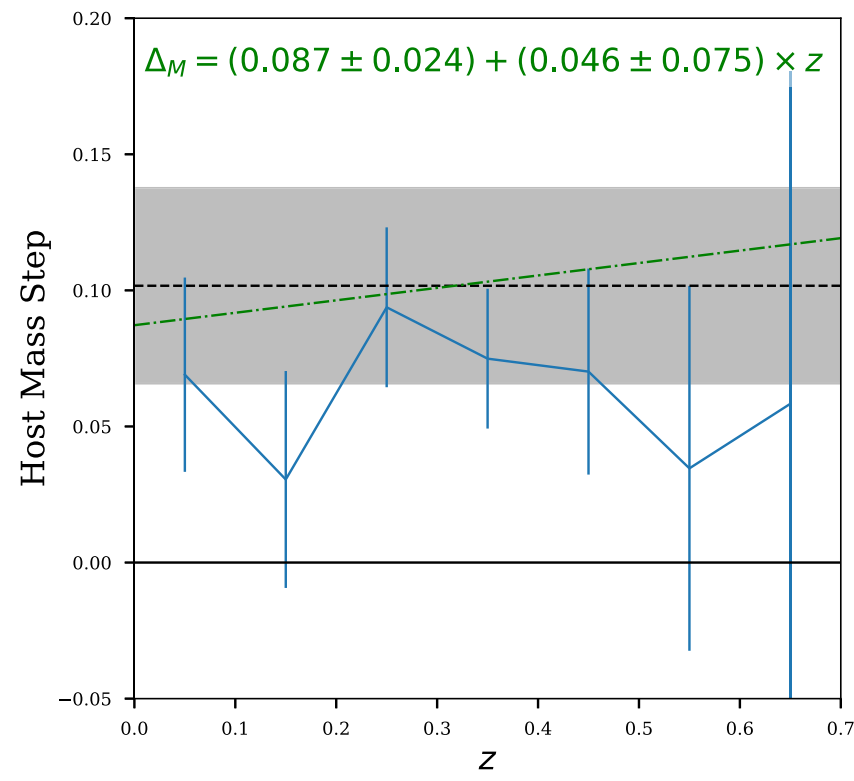

Figure 11. Evolution of the host galaxy mass step with redshift after $2.5 \sigma$ clipping to remove most $\mathrm{CC} \mathrm{SNe}$. Binned points are shown with the best-fit global mass step (black) and linear trend (green) from marginalizing over CC SNe.

SN progenitors with redshift and could contribute significantly to the systematic error budget at $z>0.5$.

We also checked for $\alpha$ evolution using the same parametric form as Equation (10) and find $\alpha(z)=0.157 \pm 0.01+z *$ $(0.018+/-0.040)$. Because we find that $\alpha$ evolution is not statistically significant, we have not included it in our systematic uncertainty budget.

\section{Cosmological Constraints from SN and CMB Data}

We first constrain $\Omega_{M}$ using the SN Ia data alone and assuming a flat $\Lambda$ CDM cosmology. We find $\Omega_{M}=0.319 \pm 0.040$, consistent with B14 $(0.295 \pm 0.034)$. These results are independent of but in good agreement with the Planck constraints on $\Omega_{M}$ $\left(\Omega_{M}=0.308 \pm 0.012\right)$.

We combine these data with CMB constraints from the Planck full-mission data (Planck Collaboration et al. 2016a). In contrast to the Planck Collaboration et al. (2014) constraints used in B14, the full-mission Planck data do not require WMAP polarization measurements. Planck provides the full likelihoods

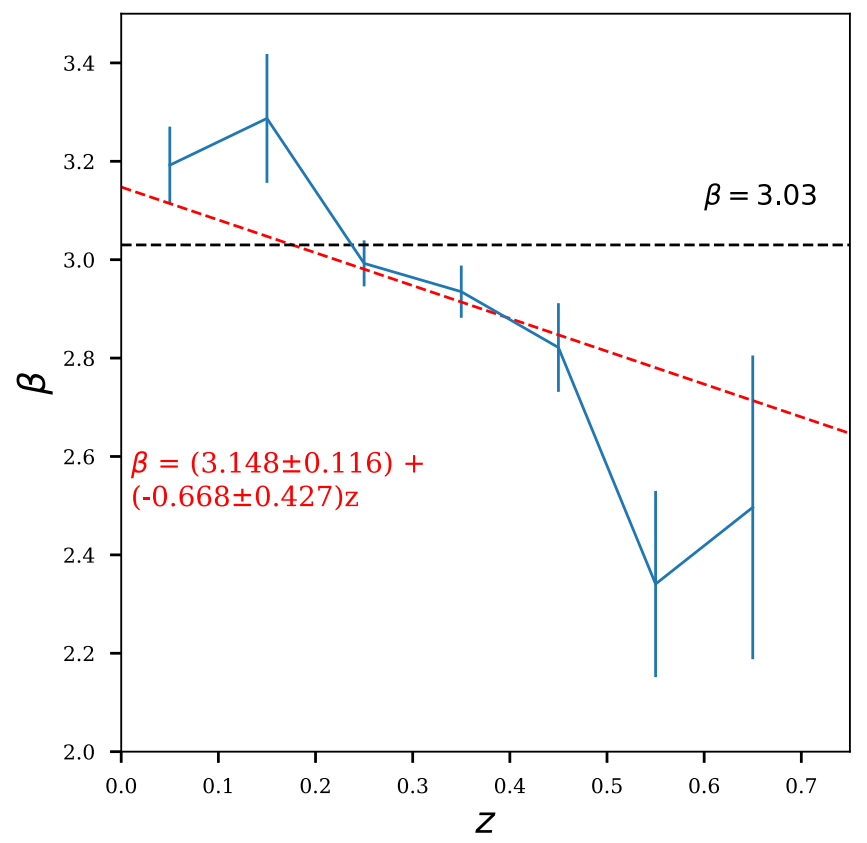

Figure 12. Binned evolution of the SALT2 nuisance parameter $\beta$ with redshift after $2.5 \sigma$ clipping to remove most CC SNe. The best-fit $\beta$ (black) and linear trend (red) are computed by marginalizing over $\mathrm{CC} \mathrm{SNe}$ with the fulllikelihood model.

for the CMB data, which can then be combined with $\mathrm{SNe}$ Ia using CosmoMC. Planck data greatly improve our constraints on $w$ using the CMB temperature power spectrum, which gives a precise constraint on the cosmic matter density at $z \sim 1090$. Constraints from a matter-dominated cosmic epoch are largely independent of an evolving or noncosmological constant dark energy, which affects cosmic evolution only at the late times probed by SN Ia and BAO measurements.

With Planck priors, we measure $w=-0.989 \pm 0.057$ (stat +sys). Systematic uncertainties on this measurement are $14 \%$ higher than statistical uncertainties (Table 6). Though we have $85 \%$ more $\mathrm{SNe}$ than $\mathrm{B} 14$ and $31 \%$ more $\mathrm{SNe}$ than $\mathrm{S} 17$, our uncertainty is approximately the same as B14 and 39\% higher than S17. There are three primary reasons for this. First, we have fewer independent surveys to reduce the photometric calibration systematic. Second, we have estimated a more conservative systematic uncertainty on the selection bias 
Table 6

Summary of Systematic Uncertainties on $w$

\begin{tabular}{lccc}
\hline \hline Error & $\Delta w^{\mathrm{a}}$ & $\Delta \sigma_{w}{ }^{\mathrm{b}}$ & Rel. to $\sigma_{w}^{\text {stat }}$ \\
\hline All sys. & 0.033 & 0.043 & 1.137 \\
Phot. cal. & 0.007 & 0.021 & 0.558 \\
Bias corr. & 0.012 & 0.019 & 0.518 \\
Mass step & 0.006 & 0.017 & 0.449 \\
Beta evol. & 0.012 & 0.016 & 0.428 \\
MW $E(B-V)$ & 0.009 & 0.015 & 0.390 \\
CC SN contam. & -0.001 & 0.012 & 0.332 \\
SALT2 model & 0.001 & 0.008 & 0.207 \\
Pec. vel. & 0.002 & 0.007 & 0.182 \\
\hline
\end{tabular}

Notes.

a Difference in measured $w$ relative to the final value of $w$ with all systematics included.

$\mathrm{b}$ The additional uncertainty added in quadrature from each source of systematic error. The statistical uncertainty on $w$ is 0.0375 .

correction than B14. Lastly, PS1 photometrically classified $\mathrm{SNe}$ have much lower $\mathrm{S} / \mathrm{Ns}$ (for PS1, the $\mathrm{S} / \mathrm{N}$ at maximum is an average of 17 for photometrically classified SNe and 39 for spectroscopically classified SNe Ia), and PS1 SNe, unlike SNLS SNe, cannot be found at $z \sim 0.7-1$.

We also use these data to constrain the two-parameter redshift evolution of $w$ using the most common parameterization:

$$
w=w_{0}+w_{a} z /(1+z) .
$$

Equation (11) is a first-order Taylor series expansion of $w$ as a function of scale factor $a$ (Linder 2003). We find $w_{0}=-0.912 \pm 0.149$ and $w_{a}=-0.513 \pm 0.826$. These constraints are slightly better than those of B14, which is due to our use of the most recent chains from Planck. We also find much tighter constraints on $w_{a}$ after combining with BAO (Section 7).

\subsection{Systematic Uncertainties on $\mathrm{w}$}

Contributions to the systematic uncertainties on $w$ are summarized in Table 6 . The photometric calibration systematic, the largest source of systematic uncertainty in most previous analyses (e.g., R14, B14), remains the largest systematic uncertainty in this work $\left(\sigma_{w}^{\text {cal }}=0.021\right)$ but is now almost the same magnitude as the selection bias. The calibration has been significantly improved by the Supercal procedure, and continued improvements will come from a new network of white dwarf standards (Narayan et al. 2016).

The second-largest systematic uncertainty is due to the selection bias $\left(\sigma_{w}^{\text {bias }}=0.020\right)$. The $\sigma_{w}^{\text {bias }}$ is dominated by the difference between the G10 and C11 scatter models and the uncertain spectroscopic selection function of the low- $z$ surveys. It may be that retraining SALT2 assuming the C11 scatter model, e.g., Mosher et al. (2014), will reduce this systematic in the future.

The systematic due to marginalizing over $\mathrm{CCSNe}$, $\sigma_{w}^{\mathrm{CC}}=0.012$, is the third-smallest systematic, nearly equal in size to the Milky Way extinction systematic and smaller than the systematics pertaining to the host mass step and $\beta$ evolution. Table 7 shows the value of $w$ measured from each classification prior and CC SN parameterization discussed in Section 4. All measurements of $w$ are within $2 \%$ of the baseline method. We note that it is likely that the NN and PSNID classifiers are more accurate than the other two classifiers used in this work. However, both NN and PSNID are directly dependent on CC SN templates and simulations for training,
Table 7

$w$ with Different Photometric Classification Priors and CC SN Models

\begin{tabular}{lrc}
\hline \hline Method & $\Delta w$ & $\Delta \sigma_{w}$ \\
\hline PSNID & $\cdots$ & $\cdots$ \\
\hline PSNID, skewed Gaussian CC model & -0.004 & 0.000 \\
PSNID, 2-Gaussian CC model & 0.018 & 0.011 \\
NN & 0.016 & 0.000 \\
GalSNID & 0.008 & 0.000 \\
Fitprob & -0.007 & 0.000 \\
Spec. $\alpha / \beta$ & -0.008 & 0.000 \\
\hline
\end{tabular}

Note. The $w$ from each CC SN model and photometric classification prior, relative to the baseline case of using PSNID classification priors and a single, $z$-dependent Gaussian to model the CC SNe. The final line is the change in $w$ when $\alpha$ and $\beta$ are fixed to the values measured from spectroscopically confirmed SNe Ia.

neither of which are likely representative of the true CC SN population (see J17 for more discussion). We include the alternative Fitprob and GalSNID classifiers, as they are less subject to the uncertainty in CC SN simulations, but we note that excluding them would significantly reduce the systematic uncertainty due to CC SN contamination.

The dispersion of measured $w$ from different BEAMS variants is nearly $\sim 25 \%$ lower than predicted by $\mathrm{J} 17$, in spite of the fact that, unlike $\mathrm{J} 17$, we did not fix $\alpha$ and $\beta$ to the values from the spectroscopic sample (except for the final variant listed in Table 7). This may be due to sample-to-sample variations but is more likely explained by tighter constraints on $\Omega_{M}$ from the full Planck chains compared to the J17 approximation $\left(\Omega_{M}\right.$ prior of $\left.0.30 \pm 0.02\right)$ and the fact that a sizable portion $(\sim 24 \%)$ of our high- $z$ data are spectroscopically classified SNe Ia. With simulations, we found that a subset of $\mathrm{SNe}$ with known types can greatly help the BEAMS method to constrain distances and SN Ia nuisance parameters (Section 5). If the amount of CCSN contamination was overestimated in $\mathrm{J} 17$, that could also help to explain the lower contamination systematic. The magnitude of the CCSN contamination systematic can be further reduced by improved validation of classifiers and a better understanding of the diversity of $\mathrm{CC} \mathrm{SNe}$ and their luminosity functions, as well as the inclusion of additional CCSN templates in classifier training, as discussed in J17. Methods for measuring robust classifications, even in the case where the training sample is biased (e.g., Revsbech et al. 2018), are also important to pursue.

\section{Cosmological Constraints with BAO and $\mathbf{H}_{0}$ Priors}

We now combine Planck (Planck Collaboration et al. 2016a) and PS $1+$ low $-z$ SNe with BAO constraints and a local prior on the value of $\mathrm{H}_{0}$ from Riess et al. (2016). The BAO feature, the evolving size of the imprint of acoustic waves on the distribution of cosmic matter, serves as a standard ruler that is independent of SN Ia measurements. The BAO scale is proportional to a combination of the angular diameter distance to a given redshift and the Hubble parameter $H(z)$ at that redshift. Following Planck Collaboration et al. (2016a), we use BAO constraints from the SDSS Main Galaxy Sample (MGS; Ross et al. 2015) and the combination of the Baryon Oscillation Spectroscopic Survey (BOSS) and CMASS survey (Anderson et al. 2014). The BAO constraints used here give measurements of the BAO scale to $z=0.15,0.32$, and 0.57 . 

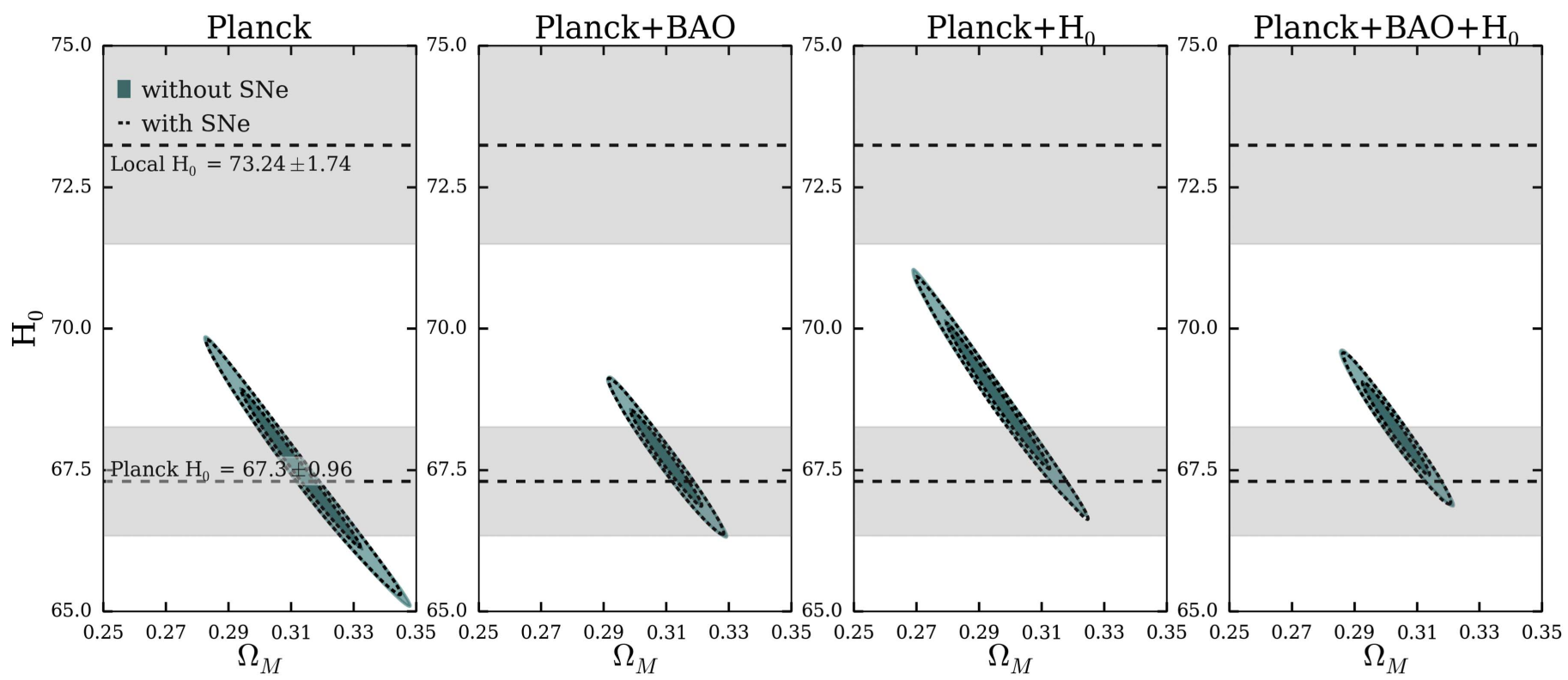

Figure 13. Discrepant constraints on $\mathrm{H}_{0}$ from $\mathrm{CMB}, \mathrm{BAO}$, and local measurements assuming $\Lambda \mathrm{CDM}$. SNe Ia disfavor a scenario in which exotic dark energy can resolve these conflicts.

Table 8

Cosmological Parameters from PS1, BAO, CMB, and $\mathrm{H}_{0}$

\begin{tabular}{|c|c|c|c|c|c|}
\hline & \multicolumn{5}{|c|}{$o-\Lambda \mathrm{CDM}$ Constraints } \\
\hline & $\Omega_{M}$ & $\overline{\Omega_{\Lambda}}$ & & $\Omega_{k}$ & $\mathrm{H}_{0}$ \\
\hline $\mathrm{PS} 1+\mathrm{Planck}+\mathrm{BAO}+\mathrm{H}_{0}$ & $0.303 \pm 0.007$ & $0.694 \pm 0.008$ & & $0.003 \pm 0.002$ & $68.682 \pm 0.694$ \\
\hline PS1+Planck & $0.330 \pm 0.045$ & $0.674 \pm 0.035$ & & $-0.004 \pm 0.011$ & $66.205 \pm 4.659$ \\
\hline $\mathrm{PS} 1+\mathrm{Planck}+\mathrm{BAO}$ & $0.310 \pm 0.007$ & $0.689 \pm 0.008$ & & $0.001 \pm 0.003$ & $67.892 \pm 0.714$ \\
\hline \multirow[t]{3}{*}{$\mathrm{PS} 1+$ Planck $+\mathrm{H}_{0}$} & $0.272 \pm 0.014$ & $0.718 \pm 0.012$ & & $0.009 \pm 0.003$ & $72.522 \pm 1.748$ \\
\hline & & & $w$-CDM Constraints & & \\
\hline & $\Omega_{M}$ & $w$ & & $\mathrm{H}_{0}$ & \\
\hline $\mathrm{PS} 1+\mathrm{Planck}+\mathrm{BAO}+\mathrm{H}_{0}$ & $0.299 \pm 0.008$ & $-1.045 \pm 0.045$ & & $69.007 \pm 0.980$ & \\
\hline PS1+Planck & $0.317 \pm 0.017$ & $-0.989 \pm 0.057$ & & $67.140 \pm 1.664$ & \\
\hline $\mathrm{PS} 1+\mathrm{Planck}+\mathrm{BAO}$ & $0.312 \pm 0.010$ & $-0.984 \pm 0.048$ & & $67.364 \pm 1.091$ & \\
\hline \multirow[t]{3}{*}{$\mathrm{PS} 1+\mathrm{Planck}+\mathrm{H}_{0}$} & $0.289 \pm 0.012$ & $-1.067 \pm 0.046$ & & $70.042 \pm 1.263$ & \\
\hline & & & $w_{a}$-CDM Constraints & & \\
\hline & $\Omega_{M}$ & $w_{0}$ & & $w_{a}$ & $\mathrm{H}_{0}$ \\
\hline $\mathrm{PS} 1+\mathrm{Planck}+\mathrm{BAO}+\mathrm{H}_{0}$ & $0.301 \pm 0.009$ & $-0.972 \pm 0.102$ & & $-0.372 \pm 0.452$ & $69.011 \pm 0.994$ \\
\hline PS1+Planck & $0.308 \pm 0.026$ & $-0.912 \pm 0.149$ & & $-0.513 \pm 0.826$ & $68.276 \pm 2.752$ \\
\hline $\mathrm{PS} 1+\mathrm{Planck}+\mathrm{BAO}$ & $0.314 \pm 0.010$ & $-0.920 \pm 0.103$ & & $-0.313 \pm 0.418$ & $67.371 \pm 1.117$ \\
\hline $\mathrm{PS} 1+\mathrm{Planck}+\mathrm{H}_{0}$ & $0.277 \pm 0.012$ & $-0.812 \pm 0.104$ & & $-1.323 \pm 0.493$ & $71.611 \pm 1.365$ \\
\hline
\end{tabular}

There is a notable internal conflict between these priors: a $3.4 \sigma$ discrepancy between local and CMB-inferred values of $\mathrm{H}_{0}$ (Riess et al. 2016; see also Bonvin et al. 2017; Casertano et al. 2017; Jang \& Lee 2017). The difference could be due to systematic uncertainties in one or both data sets (e.g., Addison et al. 2016), $>3$ neutrino species, non- $\Lambda$ dark energy, or more exotic phenomena. We show this discrepancy in Figure 13 for a standard $\Lambda \mathrm{CDM}$ cosmology (reionization optical depth $\tau=0.078$; Planck Collaboration et al. 2016b). PS $1+$ low $-z$ SNe cannot explain the disparity and therefore limit the degree to which exotic dark energy can explain the $\mathrm{H}_{0}$ tension. Throughout this section, we remain agnostic as to the source of the discrepancy and examine cosmological parameters using all probes both individually and in combination.
Following B14, we use SN data to constrain three cosmological models: the o- $\Lambda \mathrm{CDM}$ model removes the assumption of flatness $\left(\Omega_{k}=0\right)$; the $w$-CDM model allows a fixed, non-cosmological constant value of $w$; and the $w_{a}$-CDM model allows $w$ to evolve with redshift. The constraints on these three models are presented in Table 8. All measurements of $w$ and $w_{a}$ are consistent with $\Lambda \mathrm{CDM}$ (Figures 14 and 15). With $\mathrm{SNe}+\mathrm{Planck}+\mathrm{BAO}+\mathrm{H}_{0}$ constraints, we find $w=-1.045 \pm 0.045$ for the $w$-CDM model and $w_{a}=-0.372 \pm 0.452$ for the $w_{a}$-CDM model (Figure 15). With just SNe, Planck, and BAO data, we find $w=-0.984 \pm$ 0.048 for the $w$-CDM model and $w_{a}=-0.313 \pm 0.418$ for the $w_{a}$-CDM model.

Nearly all measurements of $\Omega_{k}$ are consistent with a flat universe. The lone exception is the combination of SNe, Planck, and $\mathrm{H}_{0}$. This 

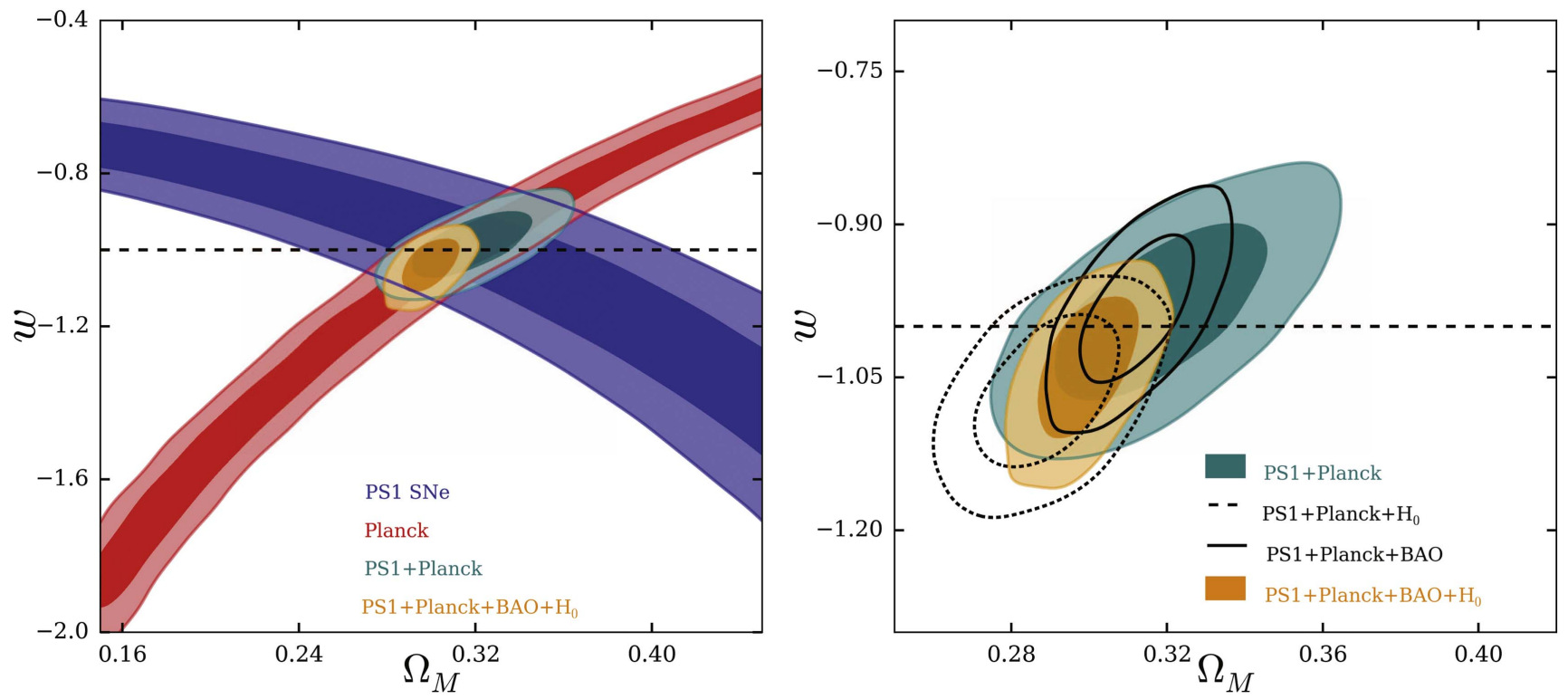

Figure 14. Constraints on $w$ and $\Omega_{M}$ from PS1+low- $z$ SNe in conjunction with other probes.

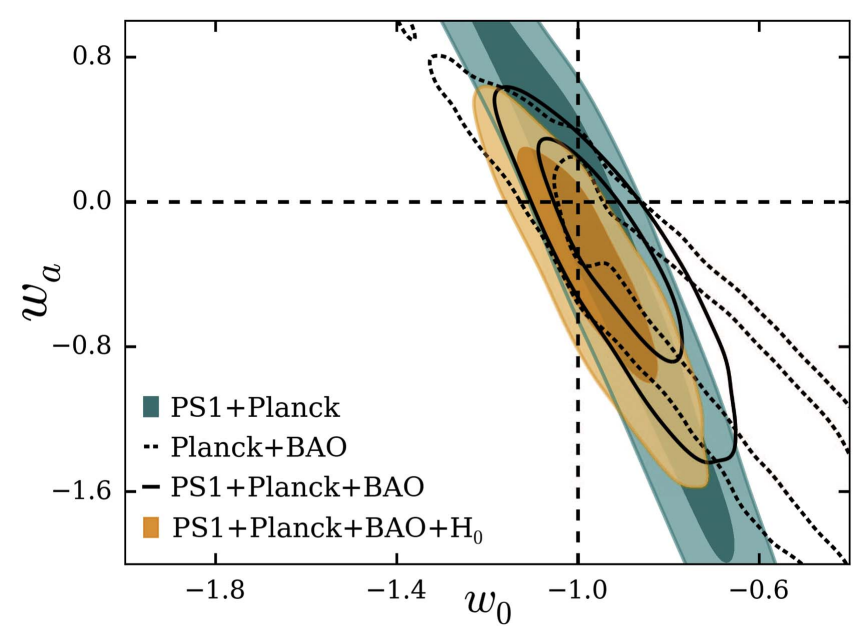

Figure 15. Constraints on $w_{0}$ and $w_{a}$ from PS1+low-z SNe, Planck, BAO, and $\mathrm{H}_{0}$.

choice of priors gives $3 \sigma$ evidence for positive curvature, but the result is entirely due to the local/CMB $\mathrm{H}_{0}$ discrepancy and becomes insignificant when $\mathrm{BAO}$ constraints are added.

As shown from the $\mathrm{H}_{0}$ measurements in Table 8, PS1+low- $z$ $\mathrm{SNe}$ and the non- $\Lambda \mathrm{CDM}$ models considered here do not explain the local/CMB $\mathrm{H}_{0}$ discrepancy. When $\mathrm{H}_{0}$ priors are omitted, all measurements of $\mathrm{H}_{0}$ are inconsistent with Riess et al. (2016) at the $\sim 2 \sigma-3 \sigma$ level and would also be inconsistent with other local measurements of $\mathrm{H}_{0}$ (Bonvin et al. 2017; Jang \& Lee 2017). When only CMB and $\mathrm{H}_{0}$ priors are included, we measure values of $\mathrm{H}_{0}$ that are consistent with Riess et al. (2016) only when allowing for positive curvature or evolving $w$. When we combine with $\mathrm{CMB}, \mathrm{H}_{0}$, and $\mathrm{BAO}$ priors, all measurements of $\mathrm{H}_{0}$ are inconsistent with Riess et al. (2016) at the $2.6 \sigma-2.8 \sigma$ level even though $\mathrm{H}_{0}$ priors are included. Therefore, SNe Ia and the models considered here do not favor a non- $\Lambda$ CDM universe or a scenario where the $\mathrm{H}_{0}$ discrepancy is due to non-cosmological constant dark energy.

\subsection{Consistency with JLA and Pantheon Results}

The binned SN Ia distances from our likelihood model are compared to the JLA sample in Figure 16 (using the correlated bins given by B14, Appendix F). The agreement is close; using weighted average Hubble residuals, PS1+low- $z$ distances are just 4 mmag fainter at $z>0.2$ compared to $z<0.2$.

Measurements of $w$ and $\Omega_{M}$ in this work show excellent agreement with B14 and the Pantheon sample (S17). For the flat $w$-CDM model, Table 9 shows the drift in the values of $w$ we measure with respect to B14 and S17. All values are consistent with B14 values to within $0.4 \sigma$. Though these measurements are correlated, as B14 used $\sim 75 \%$ of the low- $z$ $\mathrm{SNe}$ that we do (with the exception of CfA4 and CSP2), and we combine both $\mathrm{SN}$ data sets with the same $\mathrm{CMB}, \mathrm{BAO}$, and $\mathrm{H}_{0}$ data, such close agreement is encouraging.

Similarly, our measurements are consistent with those of S17 at $\lesssim 0.5 \sigma$. Though these samples are not entirely independent$34 \%$ of the SNe Ia here are included in the Pantheon samplethe samples are subject to different systematic, as well as statistical, uncertainties. The $2 \%$ discrepancy between the S17 measurement of $w$ and ours is well within the uncertainty budget of our measurement. In future work, we hope to combine our sample with the Pantheon data, as this combined sample would likely provide the best current constraints on $w$ and include just under $2000 \mathrm{SNe}$.

Though the results presented here remain subject to uncertainty in the population of $\mathrm{CCSNe}$ contaminating the $\mathrm{SN}$ data, the agreement with other measurements is encouraging. Our cosmological parameter measurements also remain consistent when using several variants as part of the BEAMS framework. The consistency of these results with measurements from spectroscopically confirmed PS1 SNe determined by S17 $(w=-0.990 \pm 0.063)$ gives us additional confidence in their robustness.

In the next few years, we also expect that additional CC SN templates and better constraints on CC SN luminosity functions will lead to even more robust simulation-based tests for this method and other similar methods. 


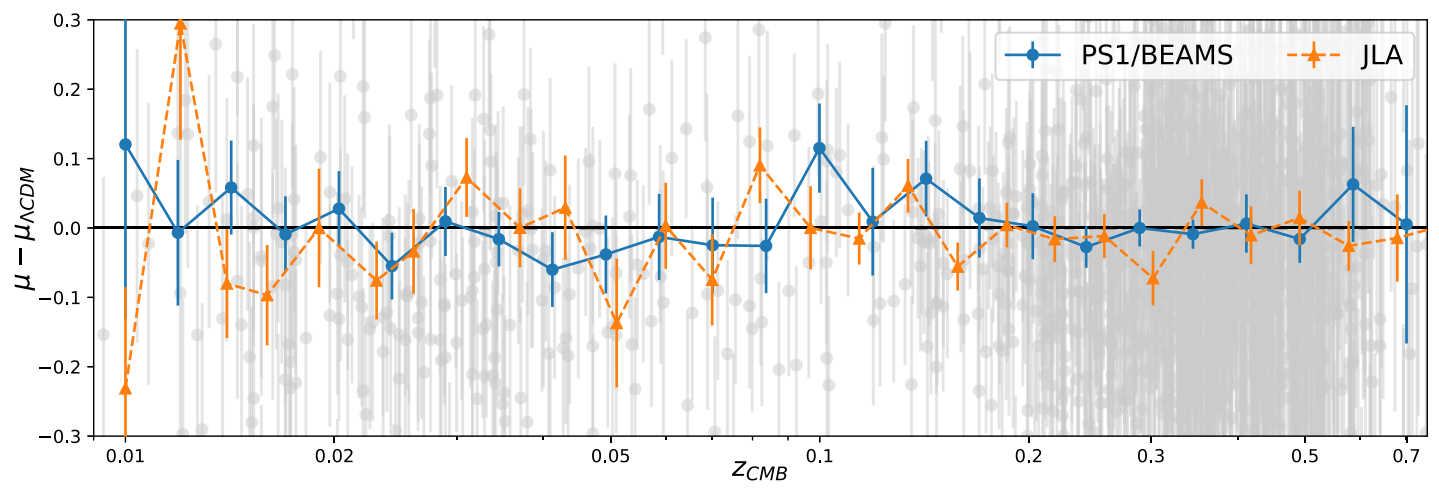

Figure 16. The PS1+low- $z$ Hubble residual diagram, with a comparison to the binned SN Ia distances given by B14. We see excellent agreement with B14 across the redshift range, with slight discrepancies at low- $z$ due to the addition of the CfA4 sample and a stronger prediction for the distance bias correction.

Table 9

Comparison to JLA and Pantheon Cosmological Constraints

\begin{tabular}{|c|c|c|c|c|c|}
\hline & \multirow{2}{*}{$\begin{array}{c}\text { This Work } \\
\quad w\end{array}$} & \multicolumn{2}{|c|}{ JLA } & \multicolumn{2}{|c|}{ Pantheon } \\
\hline & & $w$ & Diff. & $w$ & Diff. \\
\hline $\begin{array}{l}\mathrm{SNe}+\text { Planck } \\
\mathrm{SNe}+\text { Planck }+\mathrm{BAO} \\
\mathrm{SNe}+\text { Planck }+\mathrm{H}_{0} \\
\mathrm{SNe}+\text { Planck }+\mathrm{BAO}+\mathrm{H}_{0}\end{array}$ & $\begin{array}{l}-0.989 \pm 0.057 \\
-0.984 \pm 0.048 \\
-1.067 \pm 0.046 \\
-1.045 \pm 0.045 \\
w_{a}\end{array}$ & $\begin{aligned}-1.017 & \pm 0.056 \\
-1.003 & \pm 0.047 \\
-1.064 & \pm 0.051 \\
-1.038 & \pm 0.047 \\
w_{a} & \end{aligned}$ & $\begin{array}{c}0.028 \pm 0.080(0.35 \sigma) \\
0.019 \pm 0.068(0.28 \sigma) \\
0.010 \pm 0.068(0.15 \sigma) \\
0.012 \pm 0.065(0.18 \sigma) \\
\text { Diff. }\end{array}$ & $\begin{aligned} &-1.026 \pm 0.041 \\
&-1.014 \pm 0.040 \\
&-1.056 \pm 0.038 \\
&-1.047 \pm 0.038 \\
& w_{a}\end{aligned}$ & $\begin{aligned} & 0.037 \pm 0.070(0.52 \sigma) \\
& 0.030 \pm 0.063(0.48 \sigma) \\
&-0.011 \pm 0.060(0.19 \sigma) \\
& 0.002 \pm 0.059(0.03 \sigma) \\
& \text { Diff. }\end{aligned}$ \\
\hline $\begin{array}{l}\mathrm{SNe}+\text { Planck } \\
\mathrm{SNe}+\mathrm{Planck}+\mathrm{BAO} \\
\mathrm{SNe}+\mathrm{Planck}+\mathrm{H}_{0} \\
\mathrm{SNe}+\mathrm{Planck}+\mathrm{BAO}+\mathrm{H}_{0}\end{array}$ & $\begin{array}{l}-0.513 \pm 0.826 \\
-0.313 \pm 0.418 \\
-1.323 \pm 0.493 \\
-0.372 \pm 0.452\end{array}$ & $\begin{array}{l}-0.608 \pm 0.748 \\
-0.280 \pm 0.433 \\
-1.055 \pm 0.586 \\
-0.290 \pm 0.443\end{array}$ & $\begin{aligned} 0.095 & \pm 1.115(0.09 \sigma) \\
-0.033 & \pm 0.602(0.05 \sigma) \\
-0.168 & \pm 0.737(0.23 \sigma) \\
-0.073 & \pm 0.648(0.11 \sigma)\end{aligned}$ & $\begin{array}{l}-0.129 \pm 0.755 \\
-0.126 \pm 0.384 \\
-0.742 \pm 0.465 \\
-0.222 \pm 0.407\end{array}$ & $\begin{array}{l}-0.384 \pm 1.119(0.34 \sigma) \\
-0.187 \pm 0.567(0.33 \sigma) \\
-0.581 \pm 0.678(0.86 \sigma) \\
-0.150 \pm 0.608(0.25 \sigma)\end{array}$ \\
\hline
\end{tabular}

\section{Measuring $w$ without Spectroscopic Classifications}

Throughout this analysis, we have used spectroscopically confirmed SNe Ia to bolster our cosmological results. However, future samples from DES and LSST may not have a large fraction of spectroscopic classifications. Here we examine distances, nuisance parameters, and measurements of $w$ in the case where no spectroscopic classifications of PS1 $\mathrm{SNe}$ are available; we substitute photometric classifications for the available spectroscopic classifications, apply our likelihood model, and measure the resulting bias on $w$. We investigate the cases of both the full PS1 data set and the host- $z$ sample alone (only $\mathrm{SNe}$ with spectroscopic host galaxy redshifts) to determine whether our methodology can provide consistent results when spectroscopic classifications are lacking. The host- $z$ sample in particular is nearly an ideal, magnitude-limited sample, albeit with host galaxy selection biases. Of $\mathrm{SNe}$ in the full PS1 data set, $24 \%$ are spectroscopically classified SNe Ia, and $13 \%$ of SNe in the host- $z$ sample are spectroscopically classified.

When photometric classifications are used instead of spectroscopic classifications, Figure 17 shows that the binned SN Ia distances may occasionally change by $>0.05$ mag where statistical uncertainties are large. However, at $0.25 \lesssim z \lesssim 0.5$, where $~ 75 \%$ of our data lie, we see median biases less than 5 mmag for all methods. This is in agreement with predictions from J17, who found that in 25 samples of 1000 high- $z \mathrm{SNe}$, bias due to marginalizing over CC SN contamination averaged $<5 \mathrm{mmag}$ and had sample-to-sample variations of $\sim 15 \mathrm{mmag}$ in this redshift range. Although here we change at most $24 \%$ of the

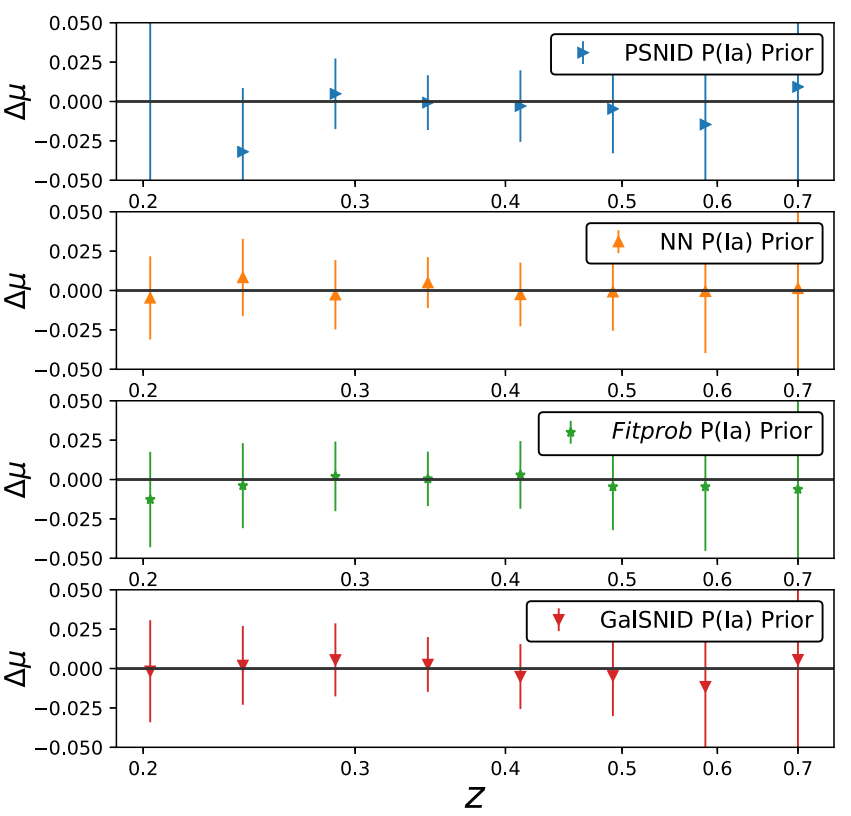

Figure 17. Changes in binned distances when spectroscopic classifications are ignored. Here $\Delta \mu$ is the bias on distance when photometric classifications are used for the $\sim 13 \%$ of the sample with spectroscopic classifications available. As we predict in J17, typical biases are $<5 \mathrm{mmag}$ for all $\mathrm{P}$ (Ia) priors at $0.2<z<0.5$ (the average is just $4 \mathrm{mmag}$ for PSNID), with occasionally larger biases in bins with higher statistical uncertainties. PSNID classifies few PS1 SNe at $\mathrm{z}<0.25$ as being likely $\mathrm{SNe} \mathrm{Ia}$, and therefore provides no meaningful constraints on distances at these redshifts. 


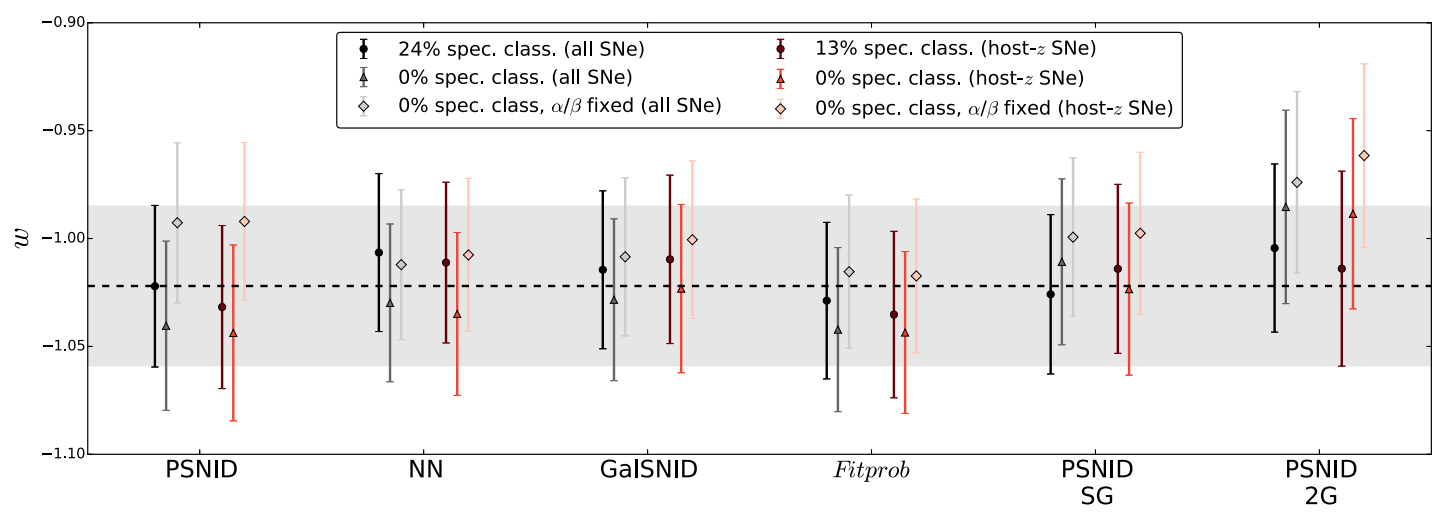

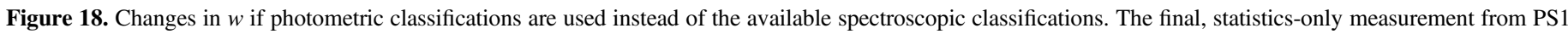

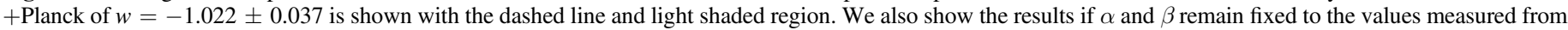

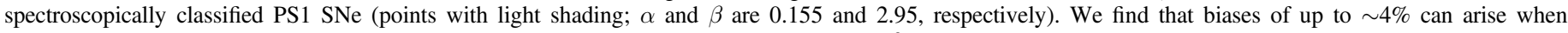
spectroscopic classifications are not available but are typically ameliorated by fixing $\alpha$ and $\beta$ to the values measured from spectroscopic samples.

classifications in the sample, the results remain broadly consistent with simulations.

In Figure 18, we examine the change in measured $w$ if spectroscopic classifications are not used. From every classifier, in both the full and host- $z$ samples, we measure a $w$ consistent with the statistical uncertainties on our best measurement of $w$, $\sigma_{w}=0.037$ (and $w$ derived from the host- $z$ sample prefers a value of $-1.032,1 \%$ lower than the full sample due to statistical fluctuations alone). However, the bias in $w$ can be nearly $\sim 4 \%$ for the least informative classifiers in this analysis (Fitprob and GalSNID), which constitutes a strong argument for including a subset of spectroscopic classifications when measuring $w$ or fixing $\alpha$ and $\beta$ to the values measured from spectroscopic samples-in this case, from PS1 spectroscopically confirmed SNe. Fixing $\alpha$ and $\beta$ can often improve the reliability of a method, an effect we show in Figure 18.

It is reassuring that many of our results appear to confirm what we predicted using simulations in J17. We see likely negative biases on $w$ when using the GalSNID and Fitprob classifiers and (typically) more consistent results after fixing $\alpha$ and $\beta$. We predicted distance biases at $0.2<z<0.5$ of $\lesssim 15$ mmag in a given SN sample, and our results here are consistent with that finding. Though not statistically significant, the $\sim$ few percent differences in nuisance parameters between spectroscopically classified SNe alone and the full sample are in the direction we would expect. With the advent of more robust classifiers and better training samples, we expect the systematic uncertainties to decrease and the reliability of simulations to improve. Even with some modest discrepancies, we see that the consistency level for nearly all methods is well within the uncertainty budget on $w$, demonstrating a promising future for SN cosmology with photometrically classified SNe.

\section{Conclusions}

The 1364 cosmologically useful, likely SNe Ia from the PS1 medium-deep fields and low- $z$ surveys constitute the largest set of SNe Ia assembled to date. Our cosmological measurement uncertainties are almost identical to those of the JLA compilation, due to the smaller redshift range and lower $\mathrm{S} / \mathrm{N}$ of the SNe Ia in our sample, but the measurements presented here are independent of the JLA data at $z>0.1$. In the future, these data can be used in conjunction with the Foundation low$z$ SN sample (Foley et al. 2018) to give independent constraints on $w$ using only the well-calibrated PS1 photometric system.
The SN light curves, host galaxy spectra, and host galaxy redshifts presented in this work are available at https://doi. org/10.17909/T95Q4X.

The PS1 SNe in this sample do not have spectroscopic classifications, necessitating a Bayesian framework that marginalizes over the CCSN population. By applying this framework, we compute binned distances from SNe Ia that are an average of just $4 \mathrm{mmag}$ fainter at $z>0.2$, compared to $z<0.2$, than JLA distances. From J17, we found that this method of marginalizing over CC SNe in a PS1-like sample will bias $w$ by a statistically insignificant $0.001 \pm 0.004$.

From these data, we find that shape- and color-corrected SNe Ia in host galaxies with $M_{*} / M_{\odot}>10 \mathrm{dex}$ are $0.102 \pm 0.017 \mathrm{mag}$ (stat+sys) brighter, on average, than those in $M_{*} / M_{\odot}<10 \mathrm{dex}$ hosts, consistent with previous measurements. We find no evidence for evolution of the mass step with redshift (e.g., Childress et al. 2014) but $\sim 1.6 \sigma$ evidence for evolution in the SALT2 $\beta$ parameter (the correlation between SN color and luminosity).

After including CMB data, we find that PS1 SN data are fully consistent with a flat $\Lambda \mathrm{CDM}$ cosmology, with $w=-0.989 \pm$ 0.057. Combining $\mathrm{SNe}$ with $\mathrm{CMB}$ and $\mathrm{BAO}$ constraints gives $w=-0.984 \pm 0.048$, and adding $\mathrm{H}_{0}$ constraints yields $w=$ $-1.045 \pm 0.045$. If we allow $w$ to be parameterized by a constant component $\left(w_{0}\right)$ and a component that evolves with redshift $\left(w_{a}\right)$, we find no evidence for a $z$-dependent value of $w$. Our constraints differ from those of $\mathrm{B} 14$ by $<0.4 \sigma$, regardless of whether CMB, $\mathrm{BAO}$, and/or $\mathrm{H}_{0}$ priors are included. They are also consistent with the constraints from Scolnic et al. (2017).

CC SN contamination is the third-smallest systematic uncertainty in this analysis and can be improved further with new SN classification algorithms and better training samples, as discussed in J17. In future work, our dominant systematicsselection biases and calibration-can be reduced by combining PS1 data with Foundation and/or SNLS and SDSS data.

In carrying out this analysis, we note that we did not blind ourselves to the cosmological results. A blinded analysis, such as that of S17, would remove any subconscious bias on the part of the authors to achieve agreement (or disagreement) with $\Lambda$ CDM cosmology. We note, however, that all of the photometry and most of the bias-correction simulations were undertaken before the cosmological results were examined. Furthermore, we have strived for consistency with previous analyses whenever possible, which serves to limit the number of qualitative choices that can be tuned to yield a preferred cosmology. Future analyses, such as DES SN Ia cosmology, will be fully blinded. As cosmology with 
photometrically classified SNe Ia becomes a more mature subject area, the authors will feel more comfortable undertaking blinded analyses.

In future years, SN samples from the DES and LSST will measure $w$ with larger, higher-S/N samples of $\mathrm{SNe}$ without spectroscopic classifications. Though CC SN contamination is the second-largest source of systematic uncertainty on $w$ in this analysis, we expect that the systematic uncertainty on $w$ from CC SN contamination will be greatly reduced in the next few years. Improvements will be due to larger samples of CC SN templates that can be used to train $\mathrm{SN}$ classification algorithms and a better understanding of the shape of the CCSN luminosity function. We hope that the methods presented here will demonstrate the robustness of measuring $w$ from photometrically classified samples as we continue to gain a better understanding of the nature of dark energy.

We would like to thank the referee for many insightful comments, which were helpful in improving this manuscript. We would also like to thank Scott Fleming and the Space Telescope Science Institute for their invaluable assistance in making the data presented in this work publicly available and user-friendly. Michael Foley also had many useful suggestions that improved this analysis.

DOJ is supported by a Gordon and Betty Moore Foundation postdoctoral fellowship at the University of California, Santa Cruz. This manuscript is based upon work supported by the National Aeronautics and Space Administration under contract No. NNG16PJ34C issued through the WFIRST Science Investigation Teams program. RJF and DS were supported in part by NASA grant 14-WPS14-0048. The UCSC group is supported in part by NSF grant AST-1518052 and by fellowships from the Alfred P. Sloan Foundation and the David and Lucile Packard Foundation to RJF. This work was supported in part by the Kavli Institute for Cosmological Physics at the University of Chicago through grant NSF PHY1125897 and an endowment from the Kavli Foundation and its founder, Fred Kavli. DS gratefully acknowledges support from NASA grant 14-WPS14-0048. DS is supported by NASA through Hubble Fellowship grant HST-HF2-51383.001, awarded by the Space Telescope Science Institute, which is operated by the Association of Universities for Research in Astronomy, Inc., for NASA under contract NAS 5-26555.

Many of the observations reported here were obtained at the MMT Observatory, a joint facility of the Smithsonian Institution and the University of Arizona. This paper uses data products produced by the OIR Telescope Data Center, supported by the Smithsonian Astrophysical Observatory. Additional data are thanks to the Anglo-Australian Telescope, operated by the Australian Astronomical Observatory, through the National Optical Astronomy Observatory (NOAO PropID: 2014B-N0336; PI: D. Jones). We also used data from observations at Kitt Peak National Observatory, National Optical Astronomy Observatory, which is operated by the Association of Universities for Research in Astronomy (AURA) under a cooperative agreement with the National Science Foundation. Also based on observations obtained with the Apache Point Observatory $3.5 \mathrm{~m}$ telescope, which is owned and operated by the Astrophysical Research Consortium.

The computations in this paper used a combination of three computing clusters. BEAMS analysis was performed using the University of Chicago Research Computing Center and the
Odyssey cluster at Harvard University. We are grateful for the support of the University of Chicago Research Computing Center for assistance with the calculations carried out in this work. The Odyssey cluster is supported by the FAS Division of Science, Research Computing Group, at Harvard University. Supernova light-curve reprocessing would not have been possible without the Data-Scope project at the Institute for Data Intensive Engineering and Science at Johns Hopkins University.

Funding for the Sloan Digital Sky Survey IV has been provided by the Alfred P. Sloan Foundation, the U.S. Department of Energy Office of Science, and the Participating Institutions. SDSS-IV acknowledges support and resources from the Center for High-Performance Computing at the University of Utah. The SDSS website is http://www.sdss.org.

SDSS-IV is managed by the Astrophysical Research Consortium for the Participating Institutions of the SDSS Collaboration, including the Brazilian Participation Group, the Carnegie Institution for Science, Carnegie Mellon University, the Chilean Participation Group, the French Participation Group, the Harvard-Smithsonian Center for Astrophysics, Instituto de Astrofísica de Canarias, the Johns Hopkins University, the Kavli Institute for the Physics and Mathematics of the Universe (IPMU)/University of Tokyo, Lawrence Berkeley National Laboratory, Leibniz Institut für Astrophysik Potsdam (AIP), Max-Planck-Institut für Astronomie (MPIA Heidelberg), MaxPlanck-Institut für Astrophysik (MPA Garching), Max-PlanckInstitut für Extraterrestrische Physik (MPE), the National Astronomical Observatory of China, New Mexico State University, New York University, the University of Notre Dame, Observatório Nacional/MCTI, the Ohio State University, Pennsylvania State University, the Shanghai Astronomical Observatory, the United Kingdom Participation Group, Universidad Nacional Autónoma de México, the University of Arizona, the University of Colorado-Boulder, the University of Oxford, the University of Portsmouth, the University of Utah, the University of Virginia, the University of Washington, the University of Wisconsin, Vanderbilt University, and Yale University.

This research makes use of the VIPERS-MLS database, operated at CeSAM/LAM, Marseille, France. This work is based in part on observations obtained with WIRCam, a joint project of CFHT, Taiwan, Korea, Canada, and France. The CFHT is operated by the National Research Council (NRC) of Canada, the Institut National des Science de l'Univers of the Centre National de la Recherche Scientifique (CNRS) of France, and the University of Hawaii. This work is based in part on observations made with the Galaxy Evolution Explorer (GALEX). GALEX is a NASA Small Explorer whose mission was developed in cooperation with the Centre National d'Etudes Spatiales (CNES) of France and the Korean Ministry of Science and Technology. GALEX is operated for NASA by the California Institute of Technology under NASA contract NAS 5-98034. This work is based in part on data products produced at TERAPIX, available at the Canadian Astronomy Data Centre as part of the CanadaFrance-Hawaii Telescope Legacy Survey, a collaborative project of NRC and CNRS. The TERAPIX team has performed the reduction of all the WIRCam images and the preparation of the catalogs matched with the T0007 CFHTLS data release. 
Funding for the DEEP2 Galaxy Redshift Survey has been provided by NSF grants AST-95-09298, AST-0071048, AST0507428, and AST-0507483, as well as NASA LTSA grant NNG04GC89G. This research uses data from the VIMOS VLT Deep Survey, obtained from the VVDS database operated by Cesam, Laboratoire d'Astrophysique de Marseille, France. The zCosmos data are based on observations made with ESO telescopes at the La Silla or Paranal Observatories under program ID 175.A-0839.

\section{Appendix \\ Simulating Evolving $x_{1}$ and $c$ Distributions}

In this appendix, we discuss the improvement to the PS1 simulations by allowing the mean simulated $x_{1}$ and $c$ to evolve with redshift. We consider the standard approach of fixed $x_{1}$ and $c$ populations insufficient for our analysis, because the PS1 host$z$ sample has redshift-dependent host galaxy properties due to our magnitude-limited host galaxy redshift follow-up program.

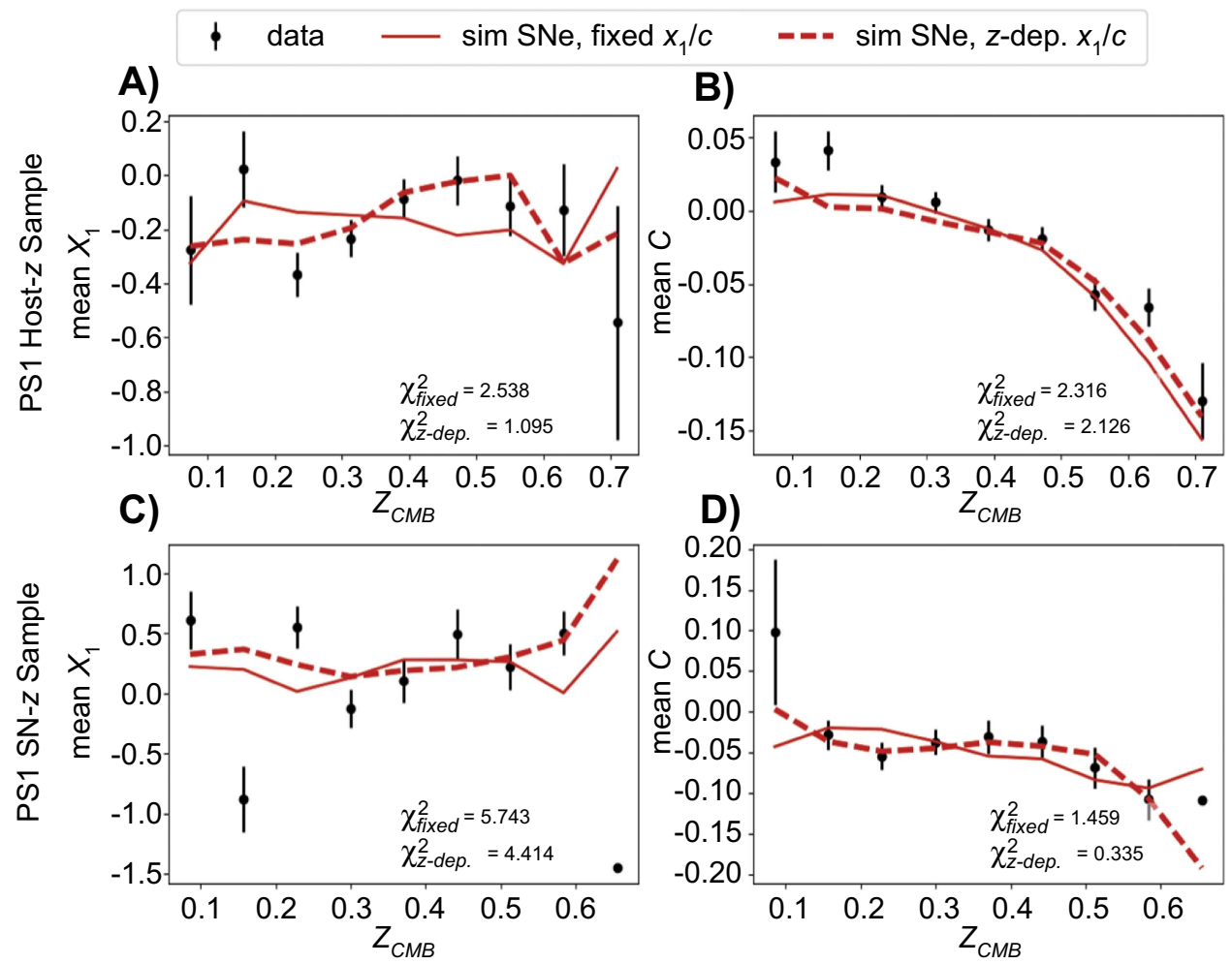

Figure 19. The $z$ dependence of $x_{1}$ and $c$. Allowing $x_{1}$ and $c$ to evolve with redshift allows simulations to better match the data.
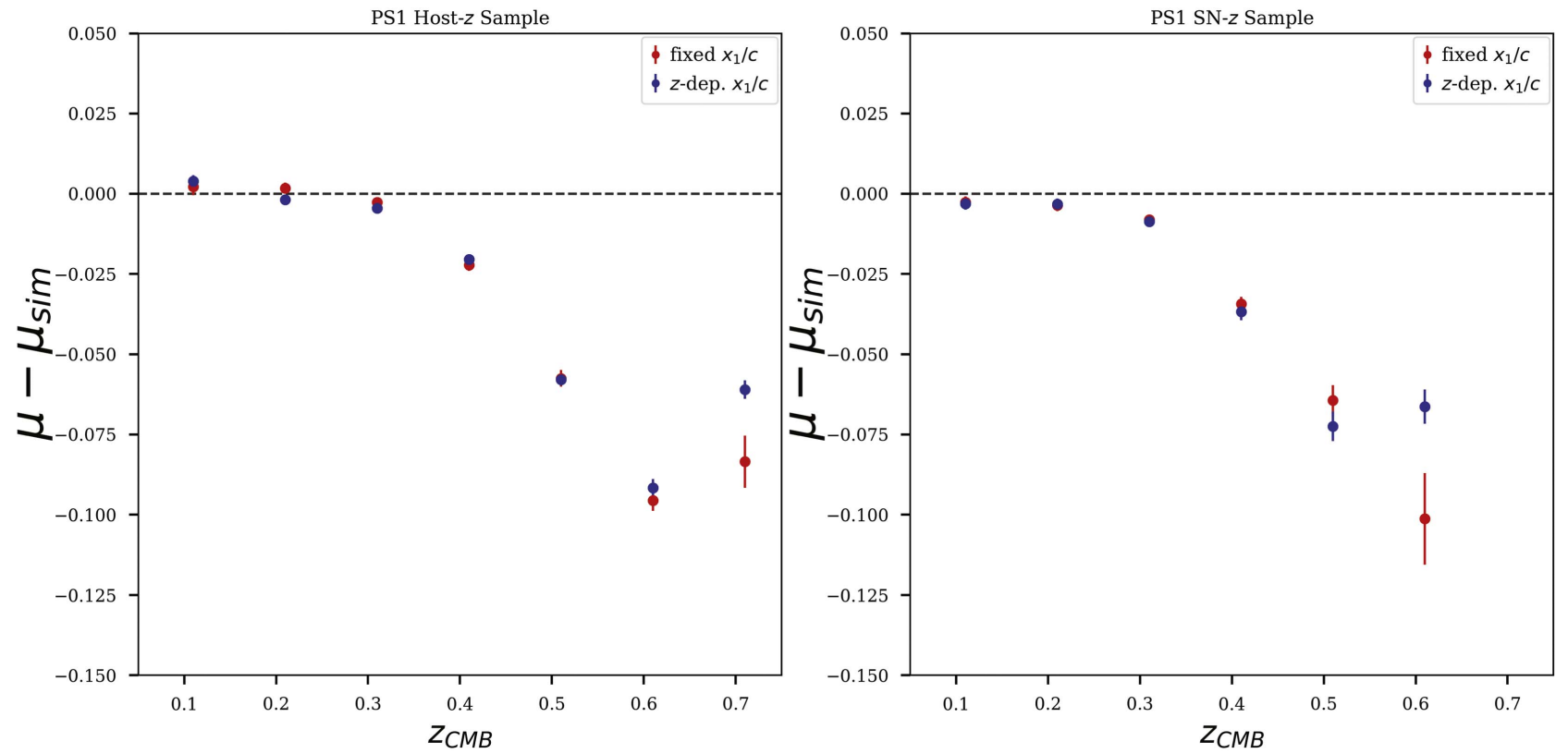

Figure 20. Change in distance bias when simulating $x_{1} / c$ distributions that evolve with redshift (G10 model). The new simulations can affect the distance bias by $\sim 0.01-0.02$ mag at high $z$. 
Similarly, the $\mathrm{SN}-z$ sample consists of $\mathrm{SNe}$ not included in the host- $z$ sample and therefore also has a $z$-dependent bias. Because $x_{1}$ and $c$ depend on host mass, their distributions change as a function of $z$ in a way that is not due only to selection biases.

Using the default simulations for the host- $z$ and $\mathrm{SN}-z$ samples from J17 and S17, respectively, we fit a third-order polynomial to the difference between the simulations and the data after binning in redshift $(\Delta z=0.05)$. We used these polynomials as inputs to SNANA, allowing them to define the intrinsic evolution of $x_{1}$ and $c$ with redshift.

Figure 19 shows the redshift dependence of the $x_{1}$ and $c$ distributions in simulations with fixed and evolving $x_{1} / c$. Though allowing $x_{1}$ and $c$ to evolve with redshift does improve the simulations, these new simulations are only a moderately better match to the data.

Figure 20 shows the difference in bias corrections using the G10 scatter model with and without $z$-dependent $x_{1}$ and $c$ populations. If $x_{1}$ and $c$ are redshift-dependent, the distance bias is slightly larger for the host- $z$ sample and smaller by up to 0.02 mag at high $z$ for the $\mathrm{SN}-z$ sample.

\section{ORCID iDs}

D. O. Jones (1D https://orcid.org/0000-0002-6230-0151

E. Berger (iD https://orcid.org/0000-0002-9392-9681

R. Kessler (iD https://orcid.org/0000-0003-3221-0419

W. S. Burgett (iD https://orcid.org/0000-0003-4401-9582

K. C. Chambers (1) https://orcid.org/0000-0001-6965-7789

P. W. Draper (iD https://orcid.org/0000-0002-7204-9802

H. Flewelling (D) https://orcid.org/0000-0002-1050-4056

M. E. Huber (10 https://orcid.org/0000-0003-1059-9603

N. Kaiser (ib https://orcid.org/0000-0001-6511-4306

N. Metcalfe (iD https://orcid.org/0000-0001-9034-4402

J. Tonry (D) https://orcid.org/0000-0003-2858-9657

R. J. Wainscoat (i) https://orcid.org/0000-0002-1341-0952

C. Waters (1D https://orcid.org/0000-0003-1989-4879

R. Kotak (i) https://orcid.org/0000-0001-5455-3653

S. J. Smartt (10) https://orcid.org/0000-0002-8229-1731

\section{References}

Addison, G. E., Huang, Y., Watts, D. J., et al. 2016, ApJ, 818, 132 Alam, S., Albareti, F. D., Allende Prieto, C., et al. 2015, ApJS, 219, 12 Amendola, L., Appleby, S., Bacon, D., et al. 2013, LRR, 16, 6 Anderson, L., Aubourg, É., Bailey, S., et al. 2014, MNRAS, 441, 24 Astier, P., Guy, J., Regnault, N., et al. 2006, A\&A, 447, 31 Bennett, C. L., Halpern, M., Hinshaw, G., et al. 2003, ApJS, 148, 1 Bertin, E., \& Arnouts, S. 1996, A\&AS, 117, 393

Betoule, M., Kessler, R., Guy, J., et al. 2014, A\&A, 568, A22

Betoule, M., Marriner, J., Regnault, N., et al. 2013, A\&A, 552, A124

Blake, C., Brough, S., Couch, W., et al. 2008, A\&G, 49, 5.19

Bohlin, R. C. 2014, arXiv:1403.6861

Bonvin, V., Courbin, F., Suyu, S. H., et al. 2017, MNRAS, 465, 4914 Campbell, H., D'Andrea, C. B., Nichol, R. C., et al. 2013, ApJ, 763, 88 Campbell, H., Fraser, M., \& Gilmore, G. 2016, MNRAS, 457, 3470 Carrick, J., Turnbull, S. J., Lavaux, G., \& Hudson, M. J. 2015, MNRAS, 450,317

Casertano, S., Riess, A. G., Bucciarelli, B., \& Lattanzi, M. G. 2017, A\&A, 599, A67

Chambers, K. C., Magnier, E. A., Metcalfe, N., et al. 2016, arXiv:1612.0556 Childress, M., Aldering, G., Antilogus, P., et al. 2013, ApJ, 770, 108 Childress, M. J., Wolf, C., \& Zahid, H. J. 2014, MNRAS, 445, 1898 Chotard, N., Gangler, E., Aldering, G., et al. 2011, A\&A, 529, L4

Colless, M., Peterson, B. A., Jackson, C., et al. 2003, arXiv:astro-ph/0306581 Conley, A., Guy, J., Sullivan, M., et al. 2011, ApJS, 192, 1

Contreras, C., Hamuy, M., Phillips, M. M., et al. 2010, AJ, 139, 519
Dai, M., \& Wang, Y. 2016, MNRAS, 459, 1819

Eisenstein, D. J., Zehavi, I., Hogg, D. W., et al. 2005, ApJ, 633, 560

Fabricant, D., Fata, R., Roll, J., et al. 2005, PASP, 117, 1411

Fioc, M., \& Rocca-Volmerange, B. 1997, A\&A, 326, 950

Flaugher, B. 2005, IJMPA, 20, 3121

Folatelli, G., Phillips, M. M., Burns, C. R., et al. 2010, AJ, 139, 120

Foley, R. J., \& Mandel, K. 2013, ApJ, 778, 167

Foley, R. J., Scolnic, D. M., Rest, A., et al. 2018, MNRAS, 475, 193

Foreman-Mackey, D., Hogg, D. W., Lang, D., \& Goodman, J. 2013, PASP, 125,306

Guy, J., Astier, P., Baumont, S., et al. 2007, A\&A, 466, 11

Guy, J., Sullivan, M., Conley, A., et al. 2010, A\&A, 523, A7

Hamuy, M., Phillips, M. M., Suntzeff, N. B., et al. 1996, AJ, 112, 2398

Hicken, M., Challis, P., Jha, S., et al. 2009a, ApJ, 700, 331

Hicken, M., Challis, P., Kirshner, R. P., et al. 2012, ApJS, 200, 12

Hicken, M., Wood-Vasey, W. M., Blondin, S., et al. 2009b, ApJ, 700, 1097

Hlozek, R., Kunz, M., Bassett, B., et al. 2012, ApJ, 752, 79

Jang, I. S., \& Lee, M. G. 2017, ApJ, 836, 74

Jha, S., Kirshner, R. P., Challis, P., et al. 2006, AJ, 131, 527

Jones, D. H., Read, M. A., Saunders, W., et al. 2009, MNRAS, 399, 683

Jones, D. O., Riess, A. G., \& Scolnic, D. M. 2015, ApJ, 812, 31

Jones, D. O., Scolnic, D. M., Riess, A. G., et al. 2017, ApJ, 843, 6

Jönsson, J., Sullivan, M., Hook, I., et al. 2010, MNRAS, 405, 535

Kaiser, N., Burgett, W., Chambers, K., et al. 2010, Proc. SPIE, 7733, 0

Kelly, P. L., Hicken, M., Burke, D. L., Mandel, K. S., \& Kirshner, R. P. 2010, ApJ, 715, 743

Kessler, R., Bassett, B., Belov, P., et al. 2010, PASP, 122, 1415

Kessler, R., Becker, A. C., Cinabro, D., et al. 2009, ApJS, 185, 32

Kessler, R., \& Scolnic, D. 2017, ApJ, 836, 56

Knights, M., Bassett, B. A., Varughese, M., et al. 2013, JCAP, 1, 039

Kunz, M., Bassett, B. A., \& Hlozek, R. A. 2007, PhRvD, 75, 103508

Lampeitl, H., Smith, M., Nichol, R. C., et al. 2010, ApJ, 722, 566

Lavaux, G., \& Hudson, M. J. 2011, MNRAS, 416, 2840

Le Borgne, D., \& Rocca-Volmerange, B. 2002, A\&A, 386, 446

Le Fèvre, O., Vettolani, G., Garilli, B., et al. 2005, A\&A, 439, 845

Lewis, A., \& Bridle, S. 2002, PhRvD, 66, 103511

Lilly, S. J., Le Fèvre, O., Renzini, A., et al. 2007, ApJS, 172, 70

Linder, E. V. 2003, PhRvL, 90, 091301

Lochner, M., McEwen, J. D., Peiris, H. V., Lahav, O., \& Winter, M. K. 2016, ApJS, 225, 31

Mink, D. J., Wyatt, W. F., Caldwell, N., et al. 2007, in ASP Conf. Ser. 376 , Astronomical Data Analysis Software and Systems XVI, ed. R. A. Shaw,

F. Hill, \& D. J Bell (San Francisco, CA: ASP), 249

Mosher, J., Guy, J., Kessler, R., et al. 2014, ApJ, 793, 16

Narayan, G., Axelrod, T., Holberg, J. B., et al. 2016, ApJ, 822, 67

Newman, J. A., Cooper, M. C., Davis, M., et al. 2013, ApJS, 208, 5

Padmanabhan, N., Schlegel, D. J., Finkbeiner, D. P., et al. 2008, ApJ, 674, 1217

Pan, Y.-C., Sullivan, M., Maguire, K., et al. 2014, MNRAS, 438, 1391

Perlmutter, S., Aldering, G., Goldhaber, G., et al. 1999, ApJ, 517, 565

Planck Collaboration, Ade, P. A. R., Aghanim, N., et al. 2014, A\&A, 571, A16

Planck Collaboration, Ade, P. A. R., Aghanim, N., et al. 2016a, A\&A, 594, 12 Planck Collaboration, Aghanim, N., Ashdown, M., et al. 2016b, A\&A, 596, A107

Rest, A., Scolnic, D., Foley, R. J., et al. 2014, ApJ, 795, 44

Revsbech, E. A., Trotta, R., \& van Dyk, D. A. 2018, MNRAS, 473, 3969

Riess, A. G., Filippenko, A. V., Challis, P., et al. 1998, AJ, 116, 1009

Riess, A. G., Kirshner, R. P., Schmidt, B. P., et al. 1999, AJ, 117, 707

Riess, A. G., Macri, L. M., Hoffmann, S. L., et al. 2016, ApJ, 826, 56

Riess, A. G., Rodney, S. A., Scolnic, D. M., et al. 2018, ApJ, 853, 126

Riess, A. G., Strolger, L.-G., Casertano, S., et al. 2007, ApJ, 659, 98

Riess, A. G., Strolger, L.-G., Tonry, J., et al. 2004, ApJ, 607, 665

Rigault, M., Aldering, G., Kowalski, M., et al. 2015, ApJ, 802, 20

Rigault, M., Copin, Y., Aldering, G., et al. 2013, A\&A, 560, A66

Roman, M., Hardin, D., Betoule, M., et al. 2017, arXiv:1706.07697

Ross, A. J., Samushia, L., Howlett, C., et al. 2015, MNRAS, 449, 835

Rubin, D., Aldering, G., Barbary, K., et al. 2015, ApJ, 813, 137

Saha, A., Wang, Z., Matheson, T., et al. 2016, Proc. SPIE, 9910, 99100F

Sako, M., Bassett, B., Becker, A. C., et al. 2014, arXiv:1401.3317

Sako, M., Bassett, B., Connolly, B., et al. 2011, ApJ, 738, 162

Schlafly, E. F., \& Finkbeiner, D. P. 2011, ApJ, 737, 103

Schlafly, E. F., Finkbeiner, D. P., Jurić, M., et al. 2012, ApJ, 756, 158

Schlegel, D. J., Finkbeiner, D. P., \& Davis, M. 1998, ApJ, 500, 525

Scodeggio, M., Guzzo, L., Garilli, B., et al. 2018, A\&A, 609, 84 
Scolnic, D., Casertano, S., Riess, A., et al. 2015, ApJ, 815, 117

Scolnic, D., \& Kessler, R. 2016, ApJL, 822, 35

Scolnic, D., Rest, A., Riess, A., et al. 2014, ApJ, 795, 45

Scolnic, D. M., Jones, D. O., Rest, A., et al. 2017, arXiv:1710.00845

Skrutskie, M. F., Cutri, R. M., Stiening, R., et al. 2006, AJ, 131, 1163

Smee, S. A., Gunn, J. E., Uomoto, A., et al. 2013, AJ, 146, 32

Stritzinger, M. D., Phillips, M. M., Boldt, L. N., et al. 2011, AJ, 142, 156

Sullivan, M., Conley, A., Howell, D. A., et al. 2010, MNRAS, 406, 782

Sullivan, M., Guy, J., Conley, A., et al. 2011, ApJ, 737, 102
Sullivan, M., Le Borgne, D., Pritchet, C. J., et al. 2006, ApJ, 648, 868

Suzuki, N., Rubin, D., Lidman, C., et al. 2012, ApJ, 746, 85

Tonry, J., \& Davis, M. 1979, AJ, 84, 1511

Tripp, R. 1998, A\&A, 331, 815

Uddin, S. A., Mould, J., Lidman, C., Ruhlmann-Kleider, V., \& Zhang, B. R. 2017, ApJ, 848, 56

Weinberg, D. H., Mortonson, M. J., Eisenstein, D. J., et al. 2013, PhR, 530, 87

Wolf, R. C., D'Andrea, C. B., Gupta, R. R., et al. 2016, ApJ, 821, 115

Zhang, B. R., Childress, M. J., Davis, T. M., et al. 2017, MNRAS, 471, 2254 